\title{
Magnetoresistive Sensor Development Roadmap (non-recording applications)
}

\begin{abstract}
Magnetoresistive (MR) sensors have been identified as promising candidates for the development of high-performance magnetometers due to their high sensitivity, low cost, low power consumption, and small size. The rapid advance of MR sensor technology has opened up a variety of MR sensor applications. These applications are in different areas that require MR sensors with different properties. Future MR sensor development in each of these areas requires an overview and a strategic guide. A MR sensor roadmap (non-recording applications) was therefore developed and made public by the Technical Committee of The IEEE Magnetics Society with the aim to provide an R\&D guide for MR sensors intended to be used by industry, government, and academia. The roadmap was developed over a three-year period and coordinated by an international effort of 22 taskforce members from 10 countries and 17 organizations, including universities, research institutes, and sensor companies. In this paper, the current status of MR sensors for non-recording
\end{abstract}

This work is supported by the Seed Funding Program for Basic Research, Seed Funding Program for Applied Research and Small Project Funding Program from the University of Hong Kong, ITF Tier 3 funding (ITS-104/13, ITS-214/14), and University Grants Committee of HK (AoE/P-04/08).

*Corresponding authors: Philip W. T. Pong (email: ppong@eee.hku.hk)

P. W. T. Pong, C. Zheng and K. Zhu are with Department of Electrical and Electronic Engineering, The University of Hong Kong, Hong Kong (e-mail: ppong@eee.hku.hk; stevenzc@ connect.hku.hk; zhuke@ connect.hku.hk).

S. Cardoso is with INESC-MN, Rua Alves Redol 9, 1000-029 Lisbon, Portugal and Departamento de Física, Instituto Superior Técnico (IST), Universidade de Lisboa-UL, Avenida Rovisco Pais, 1000- 029 Lisbon, Portugal (email: scardoso@inesc-mn.pt).

J. Chang is with Department of Power Mechanical Engineering, National Tsing Hua University, Hsinchu, Taiwan (email: jychang@ pme.nthu.edu.tw).

J. Davies and P. Eames are with NVE Corporation, Eden Prairie, Minnesota 55344, USA (email: joedavies@gmail.com; peames@nve.com).

P. P. Freitas is with INESC-MN, Rua Alves Redol 9, 1000-029 Lisbon, Portugal and INL International Iberian Nanotechnology Laboratory, Avenida Mestre José Veiga s/n, 4715-330 Braga, Portugal (email: pfreitas@inescmn.pt).

O. Kazakova is with National Physical Laboratory, Hampton Road, Teddington TW11 0LW, United Kingdom (email: olga.kazakova@npl.co.uk).

C. G. Kim is with Department of Emerging Materials Science, DGIST, Daegu 42988, Korea (email: cgkim@dgist.ac.kr).

D. Leung is with Department of Applied Physics, The Hong Kong Polytechnic University, Hung Hom, Hong Kong (email: Dennis.Leung@inet.polyu.edu.hk). applications was identified by analyzing the patent and publication statistics. As a result, timescales for MR sensor development were established and critical milestones for sensor parameters were extracted in order to gain insight into potential MR sensor applications (non-recording). Five application areas were identified, and five MR sensor roadmaps were established. These include biomedical applications, flexible electronics, position sensing (PS) and human-computer interactions (HCI), non-destructive evaluation and monitoring (NDEM), and navigation and transportation. Each roadmap was analyzed using a logistic growth model, and new opportunities were predicted based on the extrapolated curve, forecasted milestones, and professional judgement of the taskforce members. This paper provides a framework for MR sensor technology (non-recording applications) to be used for public and private $R \& D$ planning, in order to provide guidance into likely MR sensor applications, products, and services expected in the next 15 years and beyond.

S. H. Liou is with Department of Physics and Astronomy, University of Nebraska-Lincoln, Theodore P Jorgensen Hall, Lincoln, NE 68588, USA (email: sliou@unl.edu).

Alexey Ognev is with School of Natural Sciences, Far Eastern Federal University, Vladivostok, 690950, Russia (email: ognevav@gmail.com).

S. N. Piramanayagam is with Division of Physics and Applied Physics, School of Physical and Mathematical Sciences (SPMS), Nanyang Technological University, 637371, Singapore (email: prem@ntu.edu.sg).

P. Ripka is with Faculty of Electrical Engineering, Czech Technical University, Prague 166 27, Czech Republic (email: ripka@ fel.cvut.cz).

A. Samardak is with School of Natural Sciences, Far Eastern Federal University, Vladivostok, 690950, Russia and Center for Spin-Orbitronic Materials, Korea University, Seoul 02841, Korea (email: asamardak@gmail.com).

K. H. Shin is with Department of Information and Communication Engineering, Kyungsung University, Busan 608-736, Korea (email: khshin@ks.ac.kr).

S. Y. Tong and M. J. Tung are with Material and Chemical Engineering Laboratory, Industrial Technology Research Institute (ITRI), Hsinchu 300, Taiwan (email: SYTong@itri.org.tw; MJTung@itri.org.tw).

S. X. Wang is with Department of Electrical Engineering, Stanford University, Stanford, CA 94305, USA and Department of Materials Science and Engineering, Stanford University, Stanford, CA 94305, USA (email: sxwang@stanford.edu).

S. Xue is with MultiDimension Technology Corporation, No. 7 Guangdong Road, Zhangjiagang Free Trade Zone, Jiangsu 215634, China (email: song.xue@dowaytech.com).

X. Yin is with Western Digital Corporation, 44100 Osgood Rd. Fremont, CA., USA (email: Xiaolu.Yin@wdc.com). 
Keywords - magnetoresistive sensor, $\mathrm{R} \& \mathrm{D}$ guide, roadmap, smart living, Internet of Things.

\section{ACRONYMS}

AHRS Attitude and heading reference system

AMR Anisotropic magnetoresistive

AOB All-organic-based

APB All-polymeric-based

AR Augmented reality

CTC Circulating tumor cells

DOF Degree-of-freedom

EPO European Patent Office

FDA Food and drug administration

GMR Giant magnetoresistive

HCI Human-computer interaction

HPV Human Papillomavirus

IoT Internet-of-Things

MTJ Magnetic tunnel junction

MFC Magnetic flux concentrator

MEMS Micro-electro-mechanical system

MR Magnetoresistive

MCG Magnetocardiography

MEG Magnetoencephalography

NASA National Aeronautics and Space Administration

NDEM Non-destructive evaluation and monitoring

PS Position sensing

POC Point-of-care

SQUID Superconducting quantum interference device

STPO State Intellectual Property Office of China

TMR Tunnelling magnetoresistive

TIPO Taiwan Intellectual Property Office

TRL Technology readiness levels

TLC Technological life cycle

R\&D Research and development

UAV Unmanned aerial vehicle

USPTO United States Patent and Trademark Office

UUV Unmanned underwater vehicle

VR Virtual reality

\section{INTRODUCTION}

In the field of magnetic field sensing, magnetoresistive (MR) [1-4] sensors have attracted much interest owing to their high sensitivity, low cost, low power consumption, and small size [5-13]. The technological progress of MR sensors has resulted in a wide range of sensor applications, products, and services. These application areas require MR sensors with diverse properties, from high sensitivity and detectivity for biomedical applications[14-63], high mechanical flexibility and compactness for wearable/portable electronics [64-87], low power consumption and small physical dimension for position sensing (PS) [88-91] and human-computer interaction (HCI) [92-101], low cost and mass manufacturability for large-scale non-destructive evaluation and monitoring (NDEM) systems [102-122], to high accuracy and stability for navigation and transportation systems [123-141]. However, there is a lack of both an overview of the development of MR sensor applications and a strategic guide for future implementation of MR sensor technologies. These issues are resolved in this roadmap with the main scientific and technological objectives as follows:

1. To forecast MR sensor technology for the next 15 years and beyond so as to provide an R\&D guide for industry, government, and academia.

2. To provide a framework for public and private MR sensor research and development (R\&D) planning.

3. To use our expertise to predict opportunities for using MR sensors to serve society in innovative ways in the next 15 years and beyond.

The paper is structured as follows. In Section II, the roadmap development methodology is described. In Section III, the current status of MR sensors is identified, and the MR sensors development trend is summarized. In Section IV, critical sensor parameters are identified and their timelines are established, in order to gain insight into different possible sensor applications. In Section V, possible future MR sensor applications are identified, and five roadmaps are developed according to the corresponding application areas. These areas include biomedical applications, flexible electronics, PS and HCI, NDEM, and navigation and transportation. Finally, Section VI predicts the most likely future MR sensor applications.

\section{ROADMAP DEVELOPMENT METHODOLOGY}

In order to have a strategic guideline to follow, a 5-stage methodology for the roadmap development was established, as illustrated in Figure 1.

In Stage 1, the roadmap taskforce was commissioned by the Technical Committee of The IEEE Magnetic Society at the IEEE International Magnetics Conference (Intermag) 2014, in Dresden, Germany. Recruitment of taskforce members commenced.

In Stage 2, the roadmap taskforce discussed the objective and purpose of the roadmap during the $1^{\text {st }}$ taskforce meeting at the Intermag 2015, in Beijing, China. The scope and objective of the roadmap were defined, and more taskforce members were recruited.

In Stage 3, statistics of patents and publications related to MR sensors (non-recording) were analyzed. The publication data were collected from the Web of Science by keyword search. The search fields were applied only in the Title and Abstract of publications in order to exclude unrelated topics. The related patent data were obtained from four patent databases compiled by the European Patent Office (EPO), United States Patent and 
Trademark Office (USPTO), State Intellectual Property Office of China (STPO), and Taiwan Intellectual Property Office (TIPO). Based on the patent and publication data, a professional assessment of relevant MR sensor parameters was made during the $2^{\text {nd }}$ taskforce meeting at the Joint Magnetism and Magnetic Materials (MMM)/Intermag 2016, in San Diego, USA. The current status of MR sensor applications was then discussed, and critical sensor parameters for non-recording applications were identified.

In Stage 4, published articles and filed patents related to fundamental MR sensor research were reviewed. A professional assessment of critical milestones for selected sensor parameters was made during the $3^{\text {rd }}$ taskforce meeting at MMM 2016, in New Orleans, USA. Timelines for MR sensor development and for critical milestones of the sensor parameters were established and forecasted.

In Stage 5, publications related to MR sensor applications were analyzed. A professional assessment of future MR sensor applications was made according to the forecasted critical milestones for sensor parameters during the $4^{\text {th }}$ taskforce meeting at Intermag 2017, in Dublin, Ireland. Finally, a review and prediction of likely MR sensor applications, products, and services was then performed, and five roadmaps for MR sensor applications were developed.

The maturity levels of MR sensor applications, products and services were gauged by the technology readiness levels (TRL) [142]. In this paper, the classification of TRL defined by National Aeronautics and Space Administration (NASA) was adopted [143]. The TRL values of the historical MR sensor applications were analyzed using the logistic model [144, 145]. As a commonly-used growth trend curve, the logistic model has been widely utilized to describe the S-shaped feature of the technological life cycle (TLC) [142, 146, 147], which typically comprises four phases: emergence, growth, maturity, and saturation, as exhibited in Figure 2. The formula of the logistic growth curve is

$$
Y=\frac{L}{1+a e^{-b t}}
$$

where $Y$ represents the indicator related to the TRL, $t$ represents the development time, the constants $a, b$, and $L$ are the fitting parameters. In the technology emergence phase (TRL 1-2), fundamental investigation and basic research are conducted. In the technology growth phase (TRL 3-4), researches are carried out to prove the feasibility of the technology. In the technology maturity phase (TRL 5-6), model/sub-model and full-scale tests are demonstrated. In the final saturation phase (TRL 7-9), systems are validated and related products are deployed into market. In this review, we first fitted the logistic model with the TRL levels of the historical MR sensor applications so that the future trends could be predicted by extending the fitting curves beyond 2018. New opportunities were predicted by utilizing the extrapolated curve, forecasted milestones, and professional judgements on critical sensor parameters. The global vision of new MR sensor (non-recording) applications, products and services was launched out through the next 15 years and beyond.

1. MR sensor (non-recording) roadmap taskforce commissioning

by the Technical Committee of IEEE Magnetics Society at

Intermag 2014 in Dresden.

2. Recruiting taskforce members.

Stage 1

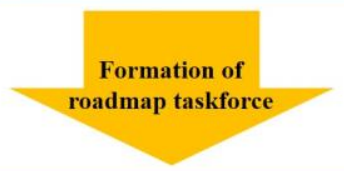

1. $1^{\text {st }}$ Roadmap taskforce meeting at Intermag 2015 in Beijing.

2. Defining roadmap missions and objectives.

\section{Stage 2}

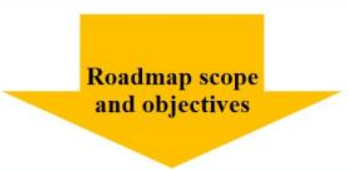

1. Identifying current status of MR sensor applications by statistical analysis on patents and publications.

2. Professional judgements on critical sensor parameters during the $2^{\text {nd }}$ taskforce meeting at Joint MMM-Intermag 2016 in San Diego.
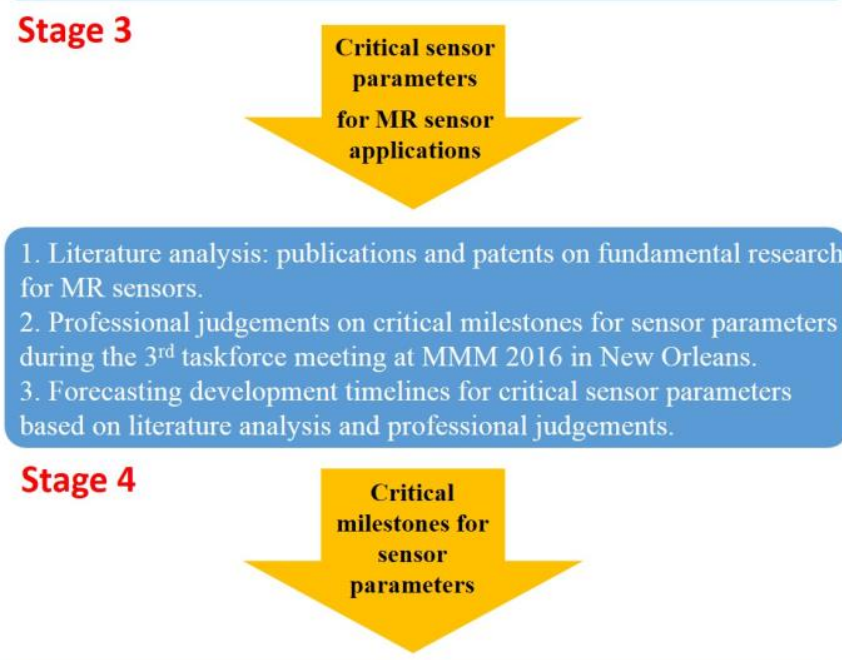

1. Literature analysis: publications on MR sensor applications.

2. Professional judgements on future applications, products and services of MR sensor technologies during the $4^{\text {th }}$ roadmap taskforce meeting at Intermag 2017 in Dublin.

3. Envisioning roadmaps for MR sensors based on literature

analysis, forecasted milestones, and professional judgements

Stage 5

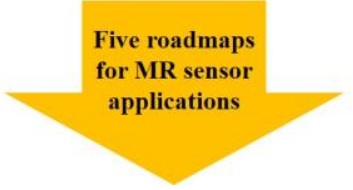

Fig. 1. Methodology for the roadmap development. 


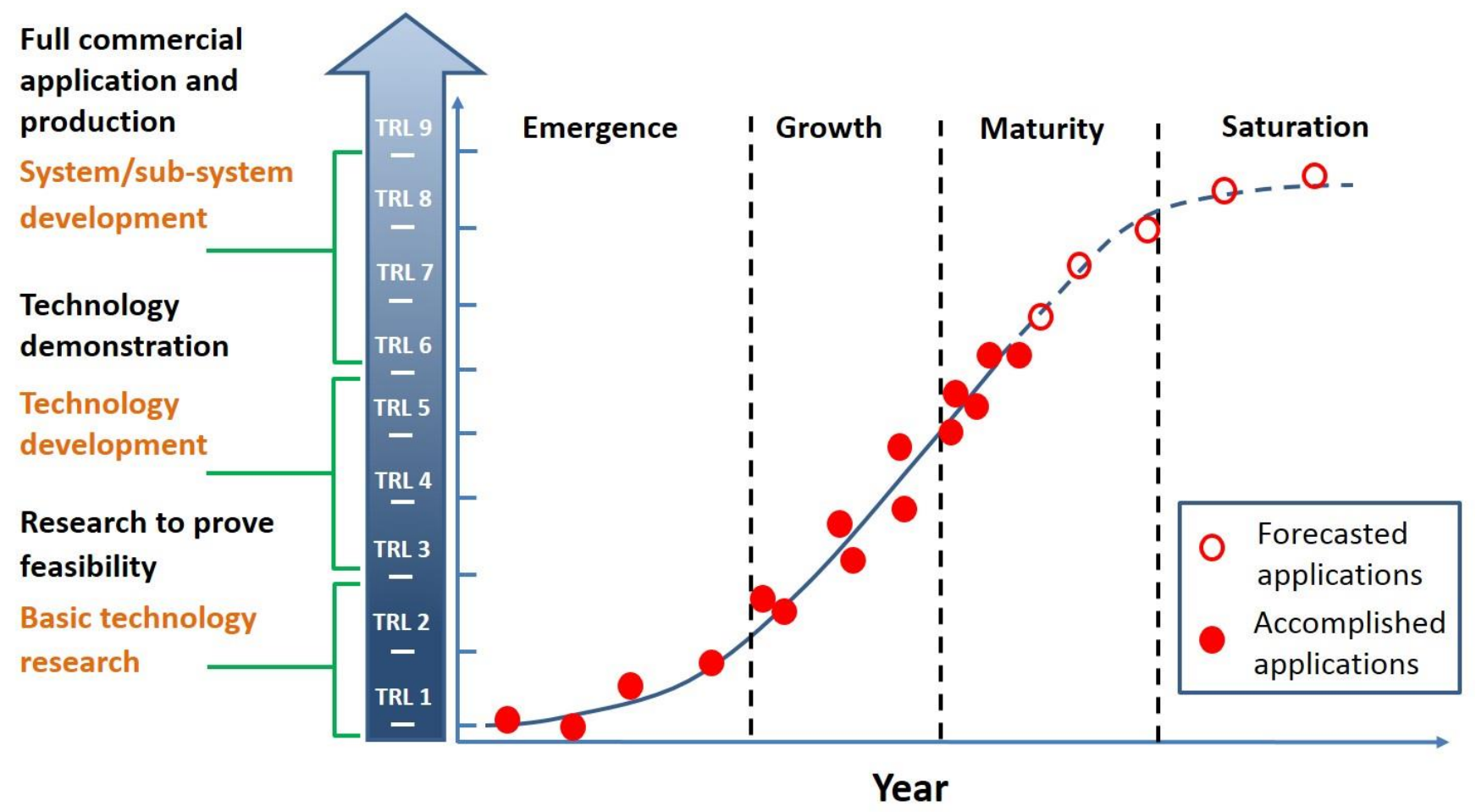

Fig. 2. Technological life cycle fitted with the logistic growth model to forecast future technological development.

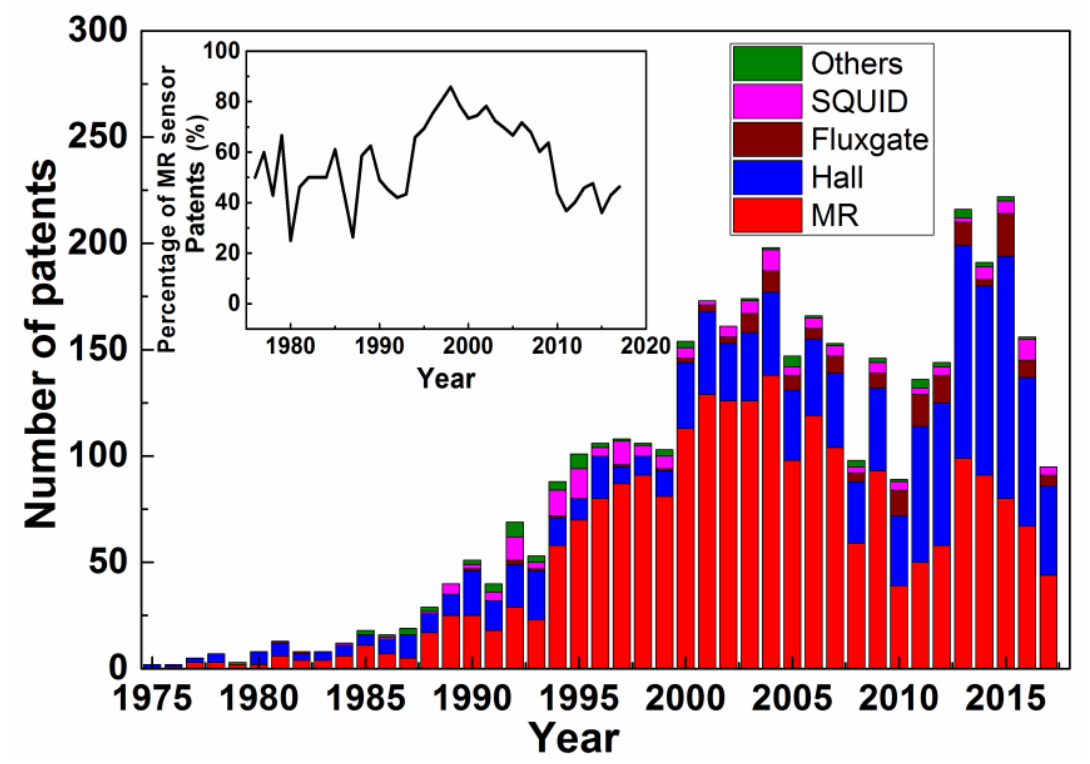

Fig. 3. Statistics of common magnetic sensors from 1975 to 2017 in the selected patent databases compiled by EPO, USPTO, STPO, and TIPO. Inset is the percentage of MR sensor patents among all types of magnetic field sensors. The list of keyword search queries for patent statistics is shown in Table I.

\section{CURRENT STATUS OF MR SENSOR APPLICATIONS}

Magnetic field detection has tremendous impact on a large variety of applications and industries $[8,9,11-13,148-152]$, which exploit a wide range of physical phenomena and principles [7, 153-167]. To obtain an overview of magnetic field sensing techniques, an analysis of statistics of common magnetic sensors from 1975 to 2017 in the selected patent databases is shown in Figure 3. To rule out any unrelated applications, the search queries were applied only in the Title and Abstract. The list of search keywords for patents statistics of magnetic field sensors is shown in Table I. Typical magnetic sensors $[13,148,149,168]$ were taken into account, including MR sensors [7, 11, 157, 169], Hall effect sensors [26, 155, 170172], fluxgates [173-177], superconducting quantum interference devices (SQUID) [156, 178-181], magneto-optical sensors [161, 182-186], search coils [187-191], magneto- 
inductive sensors [160, 192-195], magneto-impedance sensors [160, 196-199], magneto-diodes [153, 200-203], magnetotransistors [154, 204-207], and optically pumped magnetometers [158, 208-211]. As one of the most commonlyused magnetic sensors, MR sensors cover a relatively large portion of patent applications [5, 7, 10-13, 149, 169], especially during the period from 1988 to 2008, as illustrated in Figure 3. In general, MR sensors cover over $50 \%$ of the patent applications. The patent statistics trend of MR sensors (Figure 3 ) is well matched with the publication statistics curve (Figure 4). The list of search keywords for publication statistics of parallel and perpendicular anisotropic magnetoresistive (AMR), giant magnetoresistive (GMR), and tunnelling magnetoresistive (TMR) sensors is shown in Table II. Here, the perpendicular AMR refers to the planar Hall magnetoresistance/resistance effect [212-218]. The number of publications of GMR sensors exhibits an explosive growth after the discovery of GMR effect in 1988 [1,2]. After 1995, the number of publications related to TMR sensors dramatically increases and starts to exceed that of GMR sensors in 2000. The total number of publications of MR sensors reaches a peak in 2004-2006 and then shows a slight decrease (Figure 4), which is consistent with the patent trend (Figure 3).

TABLE I KEYWORD SEARCH QUERIES FOR PATENT STATISTICS OF MAGNETIC FIELD SENSORS

\begin{tabular}{|c|c|}
\hline Magnetic field sensor & Keyword \\
\hline MR sensor & $\begin{array}{l}\text { (1) "magnetoresistive" AND "magnetic" AND “sensor" } \\
\text { (2) "magnetoresistance" AND "magnetic" AND "sensor" }\end{array}$ \\
\hline Hall sensor & $\begin{array}{l}\text { (1) "Hall" AND "magnetic" AND "sensor"; } \\
\text { (2) "Hall effect" AND "magnetic" AND "sensor"; }\end{array}$ \\
\hline Fluxgate & "fluxgate" AND "magnetic" AND "sensor" \\
\hline Magneto-optical sensor & $\begin{array}{l}\text { (1) "magneto-optical" AND "magnetic" AND "sensor" } \\
\text { (2) "magnetic-optic" AND "magnetic" AND "sensor" }\end{array}$ \\
\hline $\begin{array}{l}\text { Superconducting quantum } \\
\text { interference devices }\end{array}$ & $\begin{array}{l}\text { (1) "superconducting quantum interference device" AND "magnetic" } \\
\text { AND "sensor" } \\
\text { (2) "SQUID" AND "magnetic" AND "sensor" }\end{array}$ \\
\hline Search coil & "search coil" AND "magnetic" AND "sensor" \\
\hline $\begin{array}{l}\text { Magneto- } \\
\text { inductive sensor }\end{array}$ & $\begin{array}{l}\text { (1) "magneto-inductive" AND "magnetic" AND "sensor" } \\
\text { (2) "magnetic-inductantance" AND "magnetic" AND "sensor" }\end{array}$ \\
\hline $\begin{array}{l}\text { Magneto- } \\
\text { impedance sensor }\end{array}$ & $\begin{array}{l}\text { (1) "magneto-impeditive" AND "magnetic" AND "sensor" } \\
\text { (2) "magnetic-impedance" AND "magnetic" AND "sensor" }\end{array}$ \\
\hline Magneto-diode & "magneto-diode" AND "magnetic" AND "sensor" \\
\hline Magneto-transistor & "magneto-transistor" AND "magnetic" AND "sensor" \\
\hline Optically pumped sensor & "optically pumped" AND "magnetic" AND "sensor" \\
\hline
\end{tabular}

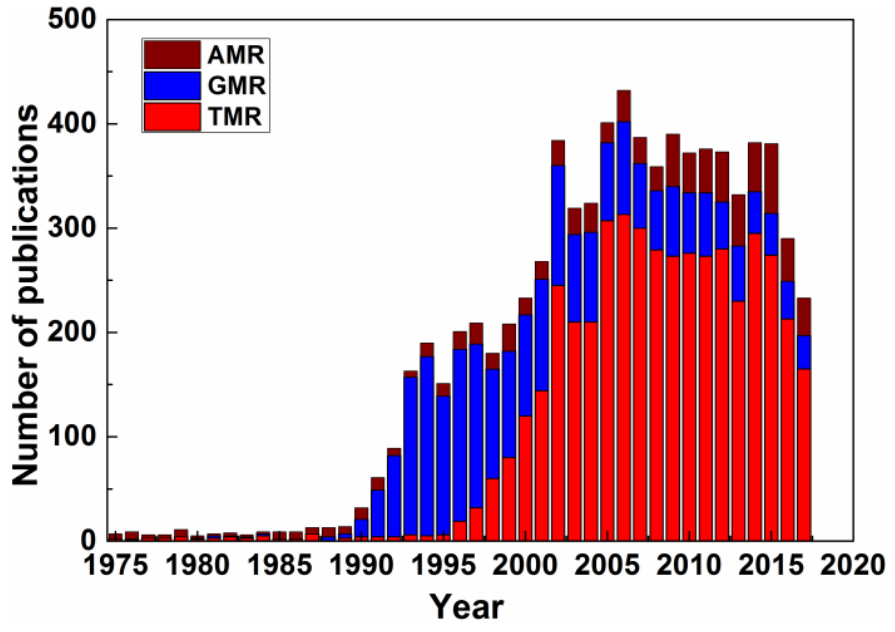

Fig. 4. Publication statistics of AMR, GMR, and TMR sensors from 1975 to 2017 in the Web of Science. The list of search keyword queries for publication statistics of AMR, GMR, and TMR sensors is shown in Table II.

TABLE II KEYWORD SEARCH QUERIES FOR PUBLICATION STATISTICS OF MR SENSORS

\begin{tabular}{|c|l|}
\hline $\begin{array}{c}\text { Magnetic field } \\
\text { sensor }\end{array}$ & \multicolumn{1}{|c|}{ Keyword } \\
\hline \multirow{2}{*}{ AMR sensor } & \begin{tabular}{l} 
(1) "anisotropic" AND "magnetoresistive" AND "sensor" \\
(2) "planisotropic" AND "magnetoresistance" AND "sensor" \\
(4) "planar Hall" AND "magnetoresistive" AND "sensor" \\
(5) "planar Hall resistance" AND "sensor" \\
\hline (1) "tunnel" AND "magnetoresistive" AND "sensor" \\
(2) "tunnel" AND "magnetoresistance" AND "sensor" \\
(3) "tunneling" AND "magnetoresistive" AND "sensor" \\
(4) "tunneling" AND "magnetoresistance" AND "sensor" \\
(5) "tunnelling" AND "magnetoresistive" AND "sensor" \\
(5) "tunnelling" AND "magnetoresistance" AND "sensor"
\end{tabular} \\
\hline (1) "giant" AND "magnetoresistive" AND "sensor" \\
(2) "giant" AND "magnetoresistance" AND "sensor"
\end{tabular}



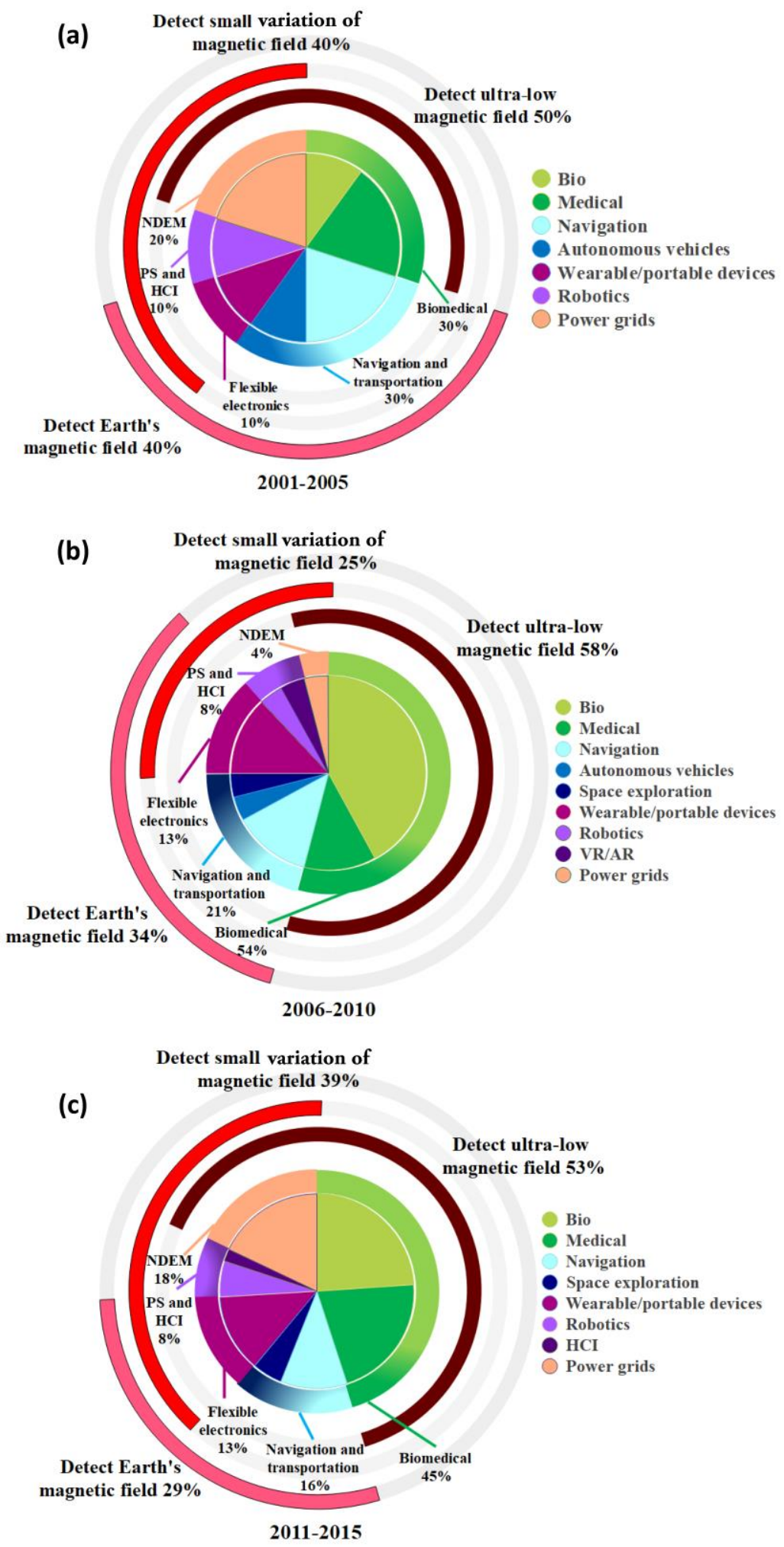

Fig. 5. Distribution of publications on MR sensor applications including biomedical applications, flexible devices, position sensing (PS) and humancomputer interactions (HCI), non-destructive evaluation and monitoring (NDEM), and navigation and transportation in the periods of (a) 2001-2005, (b) 2006-2010, and (c) 2011-2015.

Continuous endeavors from scientists and engineers have opened up various applications of MR sensor techniques [29$31,33,34,37-46,48,50,51,53-55,78,97,109,120,219-223]$ as shown in Figure 5. According to the strength of the measured field, MR sensor applications can be divided into three major categories: 1) measuring the Earth's magnetic field $(\sim \mu \mathrm{T})[123-$ 
125, 129-139, 224-233], 2) measuring small variations of magnetic field (from $\sim \mu \mathrm{T}$ to $\sim \mathrm{nT}$ ) $[107,108,110,111,113,114$, 116-121, 234], and 3) measuring ultralow magnetic field (lower than $\sim \mathrm{nT}$ ) $[16,18-21,23-31,33-35,37-40,42-44,46,48,50$, $51,53-56,222,235]$.

In the earlier applications in the period of 2001-2005 (Figure 5(a)), MR sensors were frequently used as magnetic compasses to detect Earth's magnetic field in navigation and transportation $(30 \%)[129,130,236,237]$, among which $10 \%$ were incorporated into autonomous vehicles, [126, 238] and wearable/portable devices $(10 \%)[239,240]$ as well. On the other hand, MR sensors were applied for non-destructive power-grid monitoring $(20 \%)$ [157, 241] and were utilized as sensitive magnetic probes to detect ultra-low magnetic field in biomedical applications $(30 \%)$ [18, 20, 21, 24, 27, 29].

In the period of 2006-2010 (Figure 5(b)), more MR sensors $(58 \%)$ were used to detect ultralow magnetic field owing to the improvement of their sensing performance (e.g., sensitivity, detectivity). Especially, more biomedical applications with MR sensors were explored (increased from 30\% in 2001-2005 to $54 \%$ in 2006-2011) [34-40, 42, 222]. With the development of flexible sensor substrates, a growing number of MR sensors
2001-2005 to $13 \%$ in 2006-2010) to detect Earth's magnetic field and small variations of magnetic field. A series of satellites were equipped with MR sensors for space exploration (4\%) [134, 231, 232] by virtue of their reduced size and power consumption [242-245]. MR sensors also exhibited their great compatibility with emerging technologies, such as PS and HCI $(8 \%)$ in virtual reality/augmented reality (VR/AR) [96, 246] and robotics [247].

In the period of 2011-2015 (Figure 5(c)), MR sensors continued to be widely used in the field of biomedical applications (45\%) [48, 50, 51, 53-57]. Motivated by the concept of a smart grid, more MR sensors were implemented in power grid monitoring [110, 113, 116, 119] (increased from 4\% in $2006-2010$ to $18 \%$ in $2011-2015$ ) in order to detect small variations of the magnetic field and the magnetic field generated by the currents of power cables. In order to push forward and realize MR sensor applications with existing and emerging technologies, further enhancement of MR sensor performance reflected by the critical parameters is required. Next session explores the impact of these parameters on the (1) sensitivity, (2) detectivity, (3) power consumption, (4) mechanical flexibility, and (5) robustness.

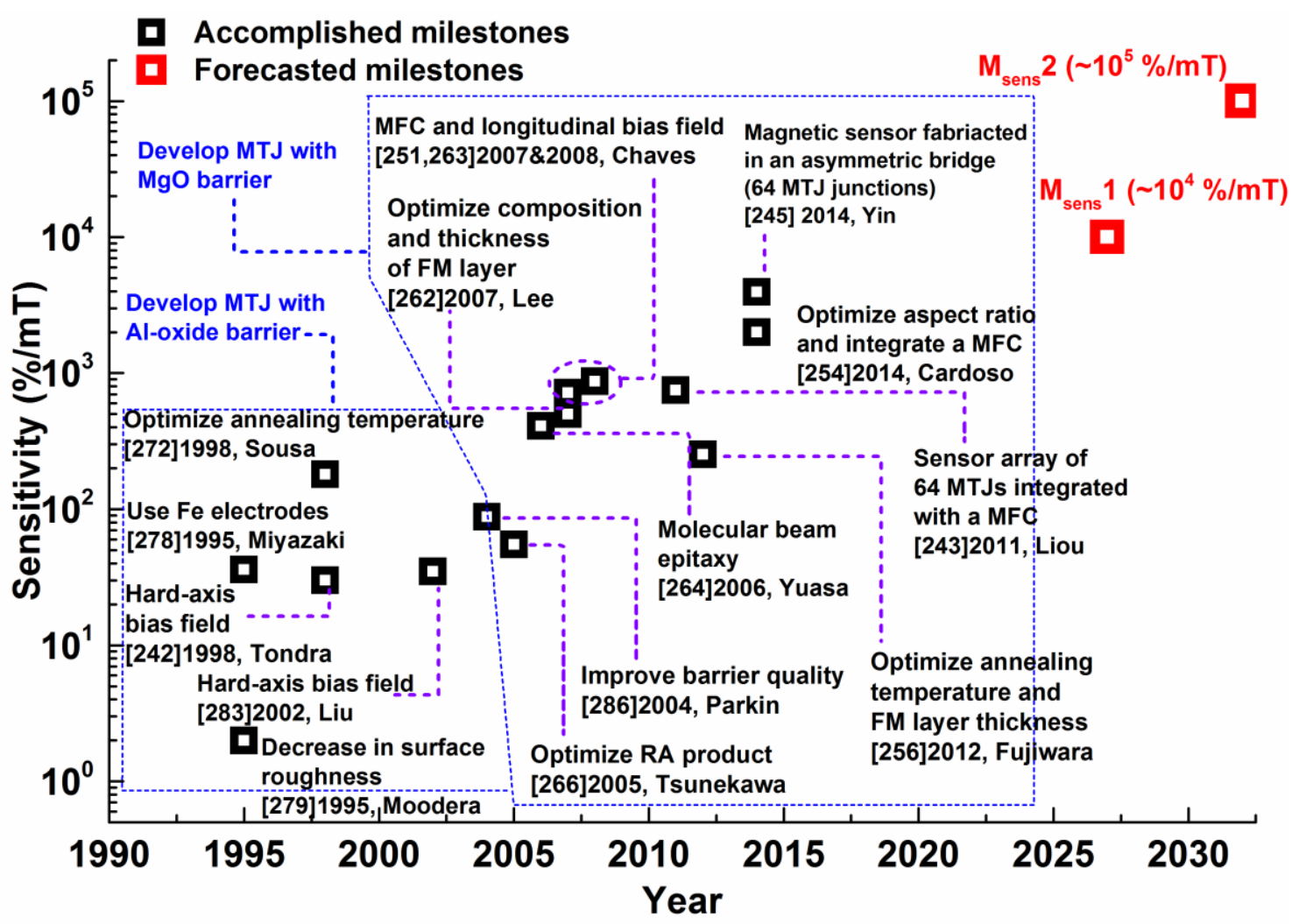

Fig. 6. Development trend for the sensitivity of MR sensors at room temperature from 1995 to 2032 .

with high tolerable tensile strain $[70,73,75]$ were integrated into wearable/portable devices [96] (increased from 10\% in 


\section{DEVELOPMENT TIMELINES FOR CRITICAL MR SENSOR PARAMETERS}

In order to gain deep insights into the technological evolution, MR sensor development timescales were established. Timelines of key sensor performance parameters including sensitivity, detectivity, power consumption, mechanical flexibility, and robustness were investigated and illustrated. Past achievements of these performance parameters were identified and their driving forces for sensor applications were discussed. Forthcoming milestones were predicted based on both the historical trends and fitted curves.

\section{A. Sensitivity}

As one of the most fundamental and critical performance parameters of MR sensors, sensitivity has exhibited a considerable growth in the last two decades [223, 243, 245, 248-266], as shown in Figure 6. The sensitivity [250, 254] of MR sensors is defined in the linear operation range of the magnetic transfer curve as

$$
S=\frac{M R}{2 \mu_{0} H_{s a t}}
$$

where $M R$ and $H_{\text {sat }}$ represent the MR ratio and saturation field, respectively. Both increased MR ratio and reduced saturation field give rise to an improved sensitivity. Large MR ratio can be obtained by selecting the thin-film materials [262, 267-271], optimizing the fabrication process [256, 272-274], and device geometry including layer thicknesses [257, 275-277]. Suppression of saturation field can be achieved by incorporating the sensors with magnetic flux concentrators (MFCs) [249, 251, 254, 263], utilizing soft ferromagnetic materials with low saturation field [262], and modifying sensor geometry [257] as well. Due to relatively high MR ratio of TMR sensors (Figure 7), researchers and engineers favor the use of TMR elements for applications requiring highly sensitive MR sensors. For the TMR sensors with an $\mathrm{AlO}_{\mathrm{x}}$ barrier during the period of 1995-2002, TMR sensors with sensitivity from several $\% / \mathrm{mT}$ to almost two hundred $\% / \mathrm{mT}$ were fabricated $[242,269,278-284]$. After replacing the $\mathrm{AlO}_{\mathrm{x}}$ barrier with the crystalline $\mathrm{MgO}$ barrier, a rapid increase of MR ratio was accomplished (Figure 7) [269, 270, 285-287], resulting in a notable enhancement of sensitivity to $300-1000 \% / \mathrm{mT}$ (Figure 6) $[245,250,251,253,255]$. By integrating MFCs into the TMR sensors, the saturation field was greatly diminished and thus the sensitivity was significantly increased [249-251, 254, 263]. Another major improvement of sensitivity was achieved by designing a sensor array with 64 MTJ junctions and incorporating the sensor array with a MFC [245]. Sensitivity as high as $3944 \% / \mathrm{mT}$ was obtained by utilizing this strategy [245]. To further improve MR sensitivity to $>10^{4} \% / \mathrm{mT}$, two technological challenges (TC) will need to be solved:

TC 1.1: accomplishment of $>1000 \%$ MR ratio at room temperature.

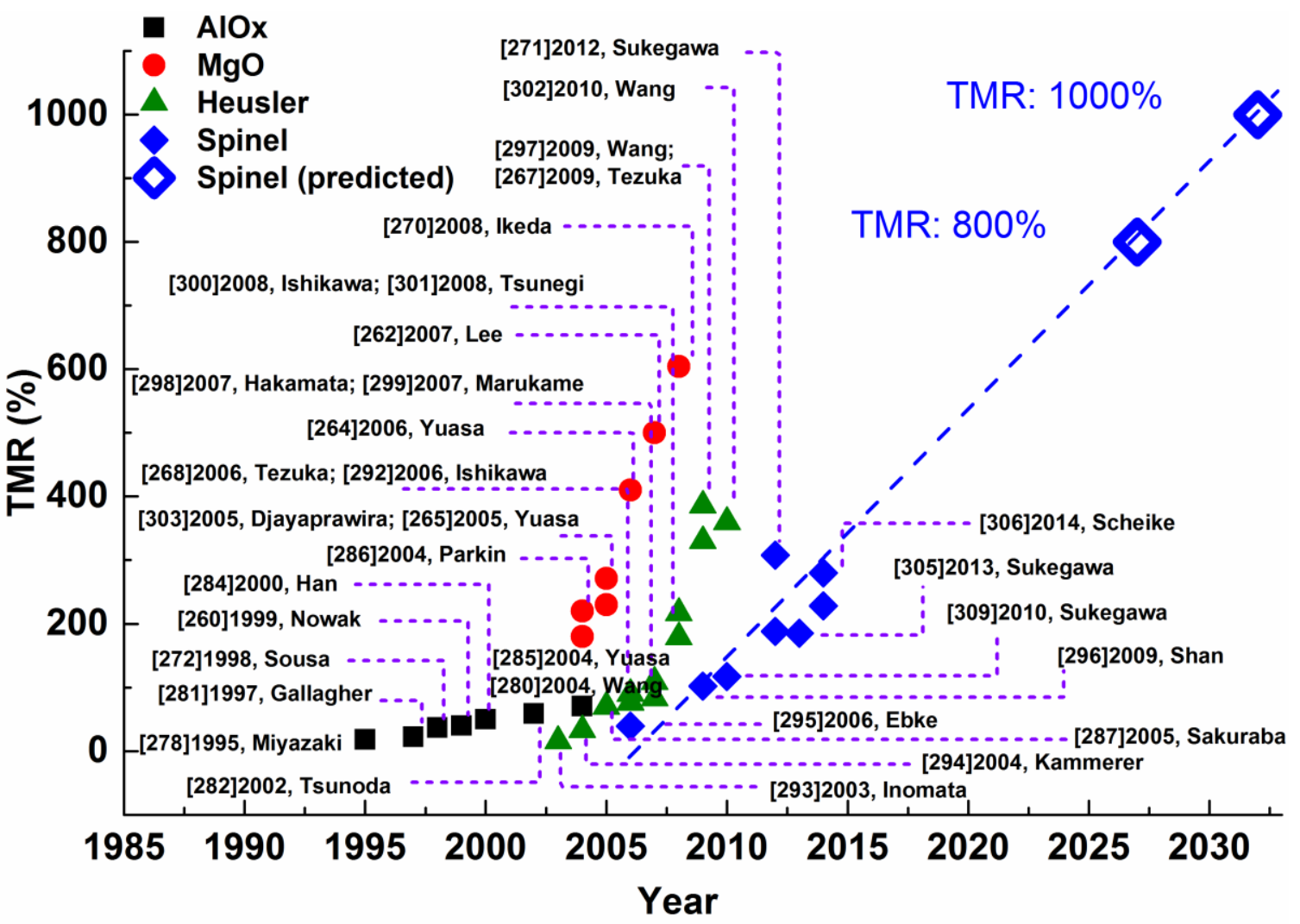

Fig. 7. Development trend of TMR ratio at room temperature for MTJs from 1995 to 2032. 
TC 1.2: accomplishment of $<0.1 \mathrm{mT}$ saturation field $2 \mu_{0} H_{\text {sat }}$ at room temperature.

For TC 1.1, the half-metallic Heusler alloy is an attractive choice of material due to high spin polarization [288-296]. As shown in Figure 7, MgO-based magnetic tunnel junction (MTJ) with Heusler alloy electrodes achieved comparable TMR ratio [267, 292, 297-302] as those MTJs with conventional ferromagnetic electrodes [264, 270, 303]. However, further enhancement of TMR was limited by the relatively large lattice mismatch between the $\mathrm{MgO}$ barrier [286] and Heusler alloy electrodes [304, 305]. This issue was resolved by replacing the $\mathrm{MgO}$ barrier with a spinel $\mathrm{MgAl}_{2} \mathrm{O}_{4}$ barrier [271, 305-308]. Compared to the $\mathrm{MgO}$ barrier, smaller lattice spacing of the $\mathrm{MgAl}_{2} \mathrm{O}_{4}$ barrier resulted in a much better lattice match of the barrier/ferromagnetic layer interface [306, 307, 309]. Furthermore, a perfectly dislocation-free interface was obtained by utilizing the cation-disorder spinel (Mg-Al-O) barrier [271, 305] where its lattice spacing was tunable through modifying the $\mathrm{Mg}-\mathrm{Al}$ compositions [305]. Therefore, a significantly enhanced TMR ratio can be expected through utilizing the lattice-tuned $\mathrm{Mg}$-Al-O barrier and optimizing the Heusler alloy electrodes. To estimate the forthcoming milestone, the historical data was fitted with a linear line and the future trend was forecasted by extrapolating the fitted line. Based on the fitting curve using the data points of spinel-based MTJs in Figure 7, 800\% TMR may be reached by $\sim 2027$, and finally $1000 \%$ TMR may be accomplished by $\sim 2032$. For TC 1.2 , the saturation field $2 \mu_{0} H_{\text {sat }}$ around $0.08 \mathrm{mT}$ was demonstrated by combining the sensor with a Conetic MFC (gain: $~ 77$ times) in 2011 [243]. In 2015, a factor of 400 times MFC was reported for an MTJ bridge [315]. In 2017, Valadeiro et al. reported a high gain ( 400 times) MFC with a double layer architecture [310]. By using this type of MFC, the authors believe that the saturation field will be further reduced from $\sim 0.08 \mathrm{mT}$ to $\sim 0.01$ $\mathrm{mT}$ in the near future. With the accomplishment of both TC 1.1 and TC 1.2, one can expect high-performance TMR sensor with sensitivity approaching $\sim 10^{4} \% / \mathrm{mT}\left(1^{\text {st }}\right.$ milestone of sensitivity: $\mathrm{M}_{\text {sens }} 1$ ) by $\sim 2027$ and $\sim 10^{5} \% / \mathrm{mT}$ ( $2^{\text {nd }}$ milestone of sensitivity: $\mathrm{M}_{\text {sens }} 2$ ) by $\sim 2032$ (see the forecasted milestones in Figure 6).

It is worth mentioning that although the linear extrapolation of MR ratio over time in Figure 7 might be optimistic, the milestone of sensitivity mentioned above can still be possibly achieved by advancing the progress of TC 1.2. At present, many experimental demonstrations already show gains of hundreds for MFCs. In fact, larger magnetic field amplification $(\sim 1000$ or even higher) can be possibly achieved by implementing the sensors inside tailor-made MFCs with their shape, dimensions and geometry (e.g., aspect ratio, the ratio of outer to inner width), material (e.g., high-permeability material) and the gap length optimized [311, 312]. As such, the final goal combining $M_{\text {sens }} 1$ and $M_{\text {sens }} 2$ is still expected.

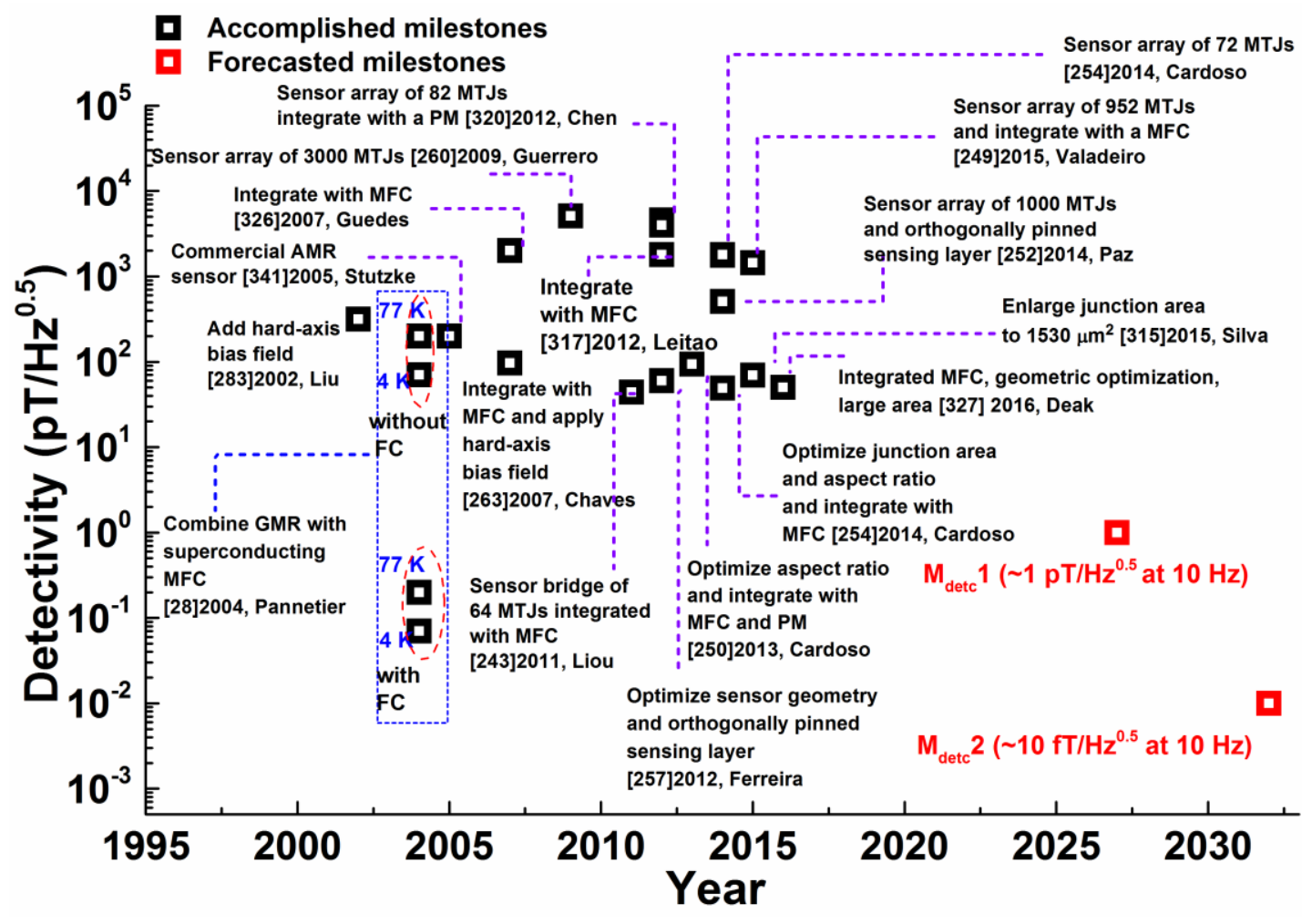

Fig. 8. Development trend of the detectivity of MR sensors at room temperature from 1995 to 2032 . 


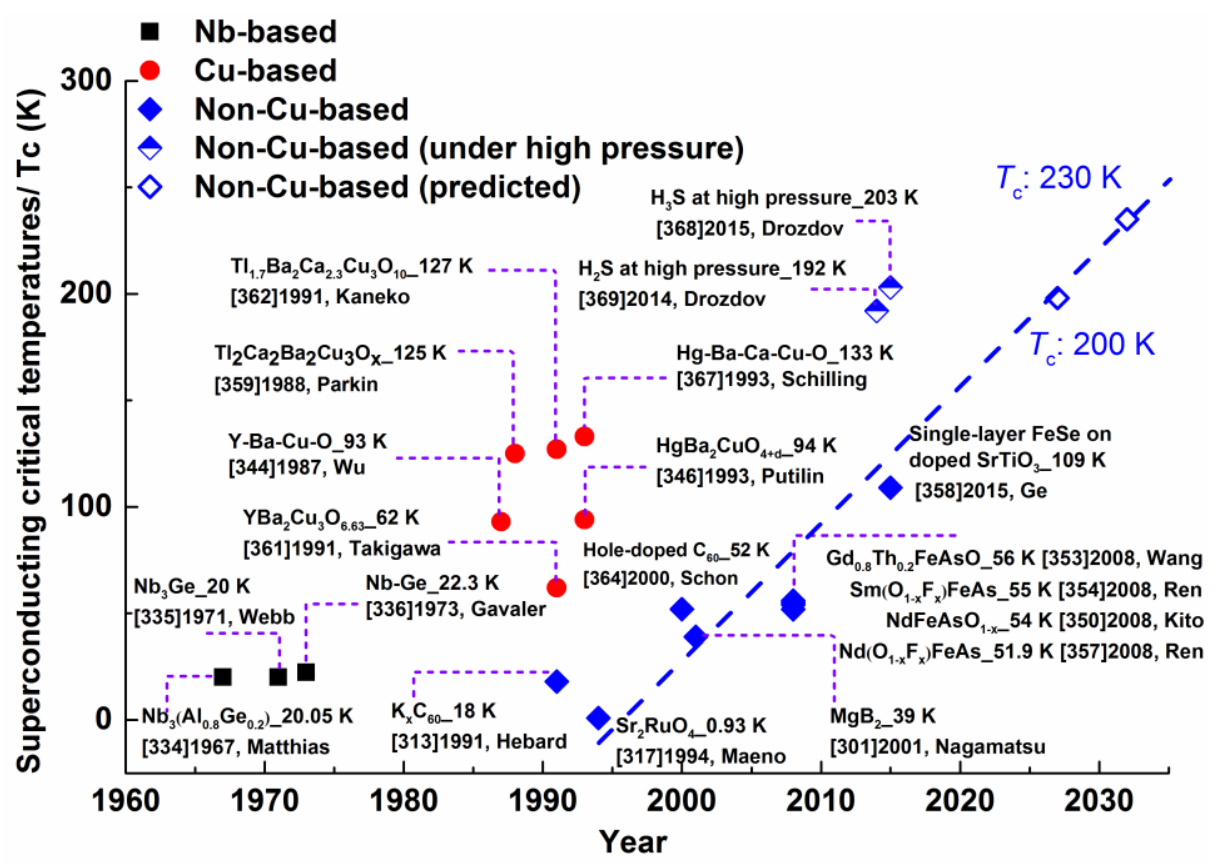

Fig. 9. Development trend of the superconducting critical temperature $\left(T_{\mathrm{c}}\right)$ of superconductors from 1967 to 2032 .

It is also worth mentioning that the noise level of a TMR sensor $\left(S_{B}\right)$ is correlated with its MR ratios. The total field noise power of a TMR sensor is given by [313]

$$
\begin{gathered}
S_{B}=\left(\frac{d B}{d V}\right)^{2}\left[S_{v}{ }^{A m p}+S_{V}{ }^{\text {shot }}+S_{v}{ }^{\text {ele. } 1 / f}\right]+S_{B}^{\text {therm.mag. }}+S_{B}{ }^{\text {mag. } 1 / f} \\
\frac{d V}{d B}=\frac{\Delta R}{R} \frac{N V_{J}}{2 B_{\text {sat }}}
\end{gathered}
$$

where $\frac{\Delta R}{R}$ is the MR ratio, $\mathrm{N}$ is the number of MTJs per leg, $V_{J}$ is the voltage drop across each MTJ, $B_{\text {sat }}$ is the free-layer saturation field, $S_{v}{ }^{A m p}, S_{V}{ }^{\text {shot }}, S_{v}{ }^{\text {elec. } 1 / f}, S_{B}{ }^{\text {therm.mag. }}$. and $S_{B}{ }^{\text {mag.1/f }}$ are amplifier noise voltage power, shot-noise voltage power, electronic $1 / f$ noise, thermal magnetic noise, and magnetic $1 / f$ noise magnetization power respectively. The overall noise level of MR sensor can be reduced by increasing MR ratio because the amplifier noise voltage power, shot-noise voltage power, and electronic 1/f noise can be suppressed by a larger MR ratio $\left(\frac{\Delta R}{R}\right.$ in Eq. (4)); however, the thermal magnetic noise and magnetic 1/f noise magnetization power do not change with the MR ratio $\left(\frac{\Delta R}{R}\right.$ in Eq. (4)). Further discussion on noise and detectivity can be found in the next section.

\section{B. Detectivity}

To fabricate high-performance MR sensors for measuring ultra-low magnetic field, researchers endeavor not only to boost their sensitivity but also to improve their detectivity which determines the smallest magnetic signal a sensor can detect [50, 222, 223, 243, 249-255, 257-260, 314-326], as shown in Figure 8. The detectivity [250] of an MR sensor is associated with its sensitivity and noise level, as expressed by

$$
D=\frac{1}{s} \sqrt{\frac{S_{V}}{V^{2}}}
$$

where $D$ is the detectivity, $S$ is the sensitivity, $V$ is the applied bias voltage and $S_{\mathrm{V}} / V^{2}$ is the normalized noise level. From Eq. 5 , both improvement of the sensitivity and suppression of the sensor noise can enhance the detectivity. As discussed in Section A, incorporation of the MR sensor array with MFCs can dramatically improve its sensitivity [245, 252], leading to a considerable increase of the sensor detectivity. On the other hand, the sensor detectivity can be greatly enhanced by reducing the sensor noise through optimization of sensor fabrication, such as enlarging the sensor area [250, 315], modifying the annealing process [243, 258, 323], and softpinning the sensing layer [249, 257]. Defect-free MR sensors with relatively large sensing area can greatly reduce the $1 / f$ noise and a sensor detectivity of $\sim 60 \mathrm{pT} / \mathrm{Hz}^{0.5}$ has been successfully demonstrated at $10 \mathrm{~Hz}$ [257]. Applying hard-axis bias field $[263,283]$ or orthogonally soft-pinning the sensing layer $[249,257]$ are effective techniques to stabilize the magnetization of the sensing layer and suppress the sensor noise. MultiDimension Technology released its highlysensitive TMR sensors (TMR9001/9002) with detectivities of $\sim 50 \mathrm{pT} / \mathrm{Hz}^{0.5}$ at $10 \mathrm{~Hz}$ in a commercial product, and $\sim 20$ $\mathrm{pT} / \mathrm{Hz}^{0.5}$ at $10 \mathrm{~Hz}$ in a larger prototype device [327]. Owing to unremitting research efforts, pT detectivities [243, 249, 252, $254,257]$ has been achieved at room temperature and fT detectivities has been demonstrated at low temperature $(77 \mathrm{~K})$ by using superconductor MFCs [28, 222]. There are other methods for reducing the low-frequency noise in MR sensors. In the modulation technique, MFCs are deposited on microelectro-mechanical systems (MEMS) flaps which are driven to oscillate at very high frequencies [328]. The advantage of modulation can only be achieved when the sensor element is responsible for most of $1 / \mathrm{f}$ noise, not the other parts of the sensor system. Moreover, it is challenging to design a successful fabrication route to combine the MEMS technology 


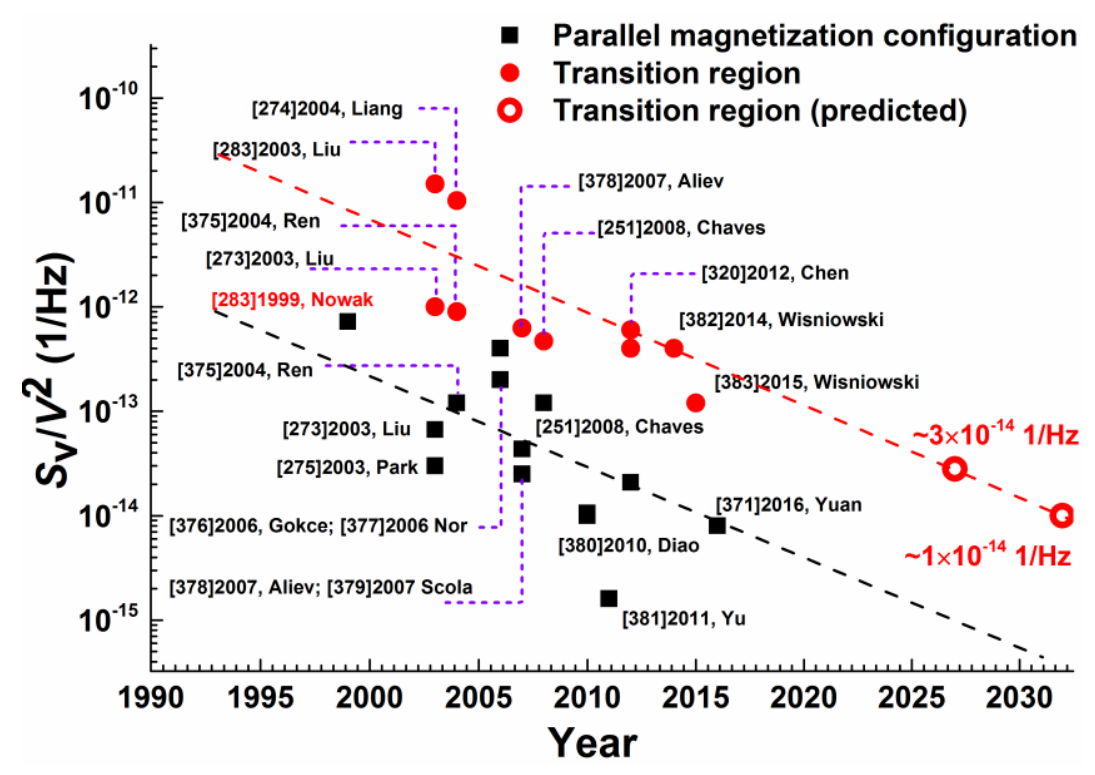

Fig. 10. Noise reduction trend at room temperature in both the magnetization-transition region and parallel magnetization configuration from 1990 to 2032.

and magnetic sensor. Though the modulation based on MEMS was presented, and several prototypes were fabricated with electro-static combs, torsionators, and cantilevers, the modulation efficiency is low [329]. In the chopping technique, chopper switches are designed for the output of MR sensors [330]. The noise characteristics of the chopper switches are dependent on charge leakage, parasitic capacitance, IC substrate coupling noise, voltage stability of the drive signal, and the external electric field sensitive electrodes [331]. All these factors need to be considered and optimized in order to suppress the noise. The methods of modulation and chopping still require research efforts to overcome these technical challenges.

To accomplish $\mathrm{fT} / \mathrm{Hz}^{0.5}$ detectivity at/near room temperature, two technological challenges (TC) have been identified:

TC 2.1: development of high-gain (>1000) MFC at/near room temperature.

TC 2.2: accomplishment of $\sim 10^{-14} 1 / \mathrm{Hz}$ normalized noise level in low frequency range (typically $<100 \mathrm{~Hz}$ ) at/near room temperature.

Regarding TC 2.1, high-temperature superconductor MFCs are required to be developed. Comparing superconducting MFCs and SQUIDs, the SQUIDs have two disadvantages. Firstly, the Josephson junction of SQUIDs is short-lived and complicated to fabricate because of poor reproducibility and low yield, and thus they are expensive [332]. Secondly, though SQUIDs comprised of ceramic HTS materials could alleviate the size, weight and power requirements, they have been found to be difficult to work with because of anisotropic electrical properties and intrinsic noise [333]. Compared to the conventional MFCs using soft ferromagnetic materials [249, 250, 254, 317, 326], superconductor MFCs exhibit a much higher gain (100-1000), as reported in [28, 222]. However, the application of superconductor MFC is restricted by its relatively low superconducting critical temperature $\left(T_{\mathrm{c}}\right)[28,222,223$, 334-366], which is far below the room temperature, as shown in Figure 9. The highest known $T_{\mathrm{c}}$ values in the $\mathrm{Cu}$-based and non-Cu-based superconductors are $133 \mathrm{~K}$ [367] and $109 \mathrm{~K}$ [358] at ambient pressure, respectively. Under high pressures, $T_{\mathrm{c}}$ values of certain superconducting materials can be notably increased [368-370] and even room-temperature superconductor MFCs can be realized. When high pressure is applied, the $T_{\mathrm{c}}$ values around $200 \mathrm{~K}$ for non-Cu-based superconductors have been achieved [368, 369], which is much higher than their $\mathrm{Cu}$-based superconductor contenders $\left(T_{\mathrm{c}} \sim 164\right.$ $\mathrm{K})$. To predict higher $T_{\mathrm{c}}$ values, a linear curve was fitted with the past data for the non-Cu-based superconductors in Figure 9. From the extrapolated curve, one expects the emergence of non$\mathrm{Cu}$-based superconductors with higher $T_{\mathrm{c}}$ than their $\mathrm{Cu}$-based superconductor contenders by $\sim 2022$. The $T_{\mathrm{c}}$ value can possibly reach $\sim 210 \mathrm{~K}$ by $\sim 2027$ and exceed $\sim 245 \mathrm{~K}$ by $\sim 2032$, which is approaching room temperature.

Regarding TC 2.2, suppression of the noise in the magnetization-transition region is the primary task because the sensor noise mainly originates from the magnetization fluctuations during operation and its magnitude is considerably larger than that of the electrically originated noise (as exhibited in the parallel magnetization configuration) $[255,273,371$ 383], as shown in Figure 10. Since operation region of MR sensors is where the magnetization of the sensing layer undergoes a transition, we predict the noise reduction trend by fitting and extrapolating the noise data for the magnetizationtransition region with a linear line. Normalized noise level around $\sim 3 \times 10^{-14} 1 / \mathrm{Hz}$ can be expected by $\sim 2027$ and one can estimate noise level to go down to the order of $\sim 1 \times 10^{-15} 1 / \mathrm{Hz}$ in approximately 15 years (i.e., 2032). Considering the forecasted accomplishments for both sensitivity and noise level 
in the following 15 years, one expects that a detectivity of $\sim 1$ $\mathrm{pT} / \mathrm{Hz}^{0.5}\left(1^{\text {st }}\right.$ Milestone of detectivity: $\left.\mathrm{M}_{\text {detc }} 1\right)$ can be achieved by $\sim 2027$. Incorporating MR sensors with near-roomtemperature superconductor MFC (gain: 1000 times), a detectivity of $\sim 10 \mathrm{fT} / \mathrm{Hz}^{0.5}$ ( $2^{\text {nd }}$ Milestone of detectivity: $\mathrm{M}_{\mathrm{detc}}$ 2 ) is expected by $\sim 2032$ (see the forecasted milestones in Figure 8).

It should be noted that the expected detectivity may not be achievable without the deployment of magnetic shielding because the external background magnetic field noise may render the low-field detectivity useless. Magnetic shielding can effectively eliminate background field noise and facilitate lowfield detection [384-395]. Magnetic shielding with high shielding effectiveness can be fabricated with soft magnetic materials such as Conetic alloy [395, 396] and multi-layered structures [397-399]. The field reduction exceeded $25 \mathrm{~dB}$ for combined active and passive shields in 2003 [400]. In 2007, a shielding factor of $6 \times 10^{6}$ was measured in a nested set of three shields, and a shielding factor of up to $10^{13}$ was predicted when five shields were used [401]. In the work of Komack's group [402], a magnetometer with single-channel sensitivity of 0.75 $\mathrm{ft} / \mathrm{Hz}^{0.5}$ was demonstrated by using a ferrite shield, limited only by the magnetization noise of ferrite and photon shot noise. In the high-temperature superconducting area, shielding factors as high as $95 \%$ were observed for three-layer hybrid shielding structures in 2016 [403]. A group reported that $98 \%$ attenuation of the magnetic field was achieved by more than five layers of the coated conductor tape wound with the same orientation and angle to cover the gaps of an inner layer achieves in 2018 [385]. Some researchers are now making use of computational intelligence to optimize a series of shielding parameters such as material, shape, thickness, and the number of layers for a higher shielding effectiveness [404-406].

Besides, it is worth mentioning that the influence of MR ratio and noise are discussed separately in Section IV(A) and (B), respectively. The discussion in Section IV(A) on sensitivity and MR ratio is purely based on \%/mT as derived from Eq. 2 which does not take into account the noise. The detailed discussion on noise is provided in Section IV(B) which elaborates on detectivity from the point of view of noise level $\left(\mathrm{T} / \mathrm{Hz}^{0.5}\right)$. In fact, a good MR sensor needs both good MR ratio and low noise level. Now the researchers are working on the realization of the ultra-sensitive and high-resolution MR sensors by reducing their intrinsic noise without sacrificing MR ratios. The authors in Ref. [407] worked on a TMR device with CoFeB-MgO$\mathrm{CoFeB}$ structures with $\mathrm{MR}$ ratios up to $600 \%$ at room temperature. They showed that the voltage-induced magnetic anisotropy modulation could be used to control and reduce magnetic noise in TMR sensors with perpendicular anisotropy. The magnetic noise was reduced by around one order of magnitude. In Ref. [320], the yoke-shaped TMR sensors based on MgO-barrier MTJs were designed. Their field sensitivity was up to $27 \% / \mathrm{mT}$, while the field detectivity reached 3.6 $\mathrm{nT} / \mathrm{Hz}^{0.5}$ at $10 \mathrm{~Hz}$ and $460 \mathrm{pT} / \mathrm{Hz}^{0.5}$ at $1 \mathrm{k} \mathrm{Hz}$ through designing a nearly-perpendicular configuration of two ferromagnetic electrodes. The TMR sensors fabricated with electron-beam evaporated $\mathrm{MgO}$ barriers can provide about an order of magnitude improvement in their signal-to-noise ratio compared to the conventional sputtered $\mathrm{MgO}$ tunnel barriers [380]. Frequency noise was investigated in $\mathrm{MgO}$ double-barrier MTJs with TMR ratios up to $250 \%$ at room temperature, and the research disclosed that the double-barrier MTJs were useful for improving the signal-to-noise ratio compared to single-barrier MTJs under low bias. These methods are critical for the overall improvement in the field detectivity of MR-sensor devices and their applications.

\section{Operational performance (power consumption, mechanical flexibility, robustness)}

In addition to high-performance sensing, MR sensors have other desirable capabilities, including low power consumption [242-245], high mechanical flexibility [83, 85], and high robustness [127, 128, 134, 135], as shown in Figure 11.

Power consumption is critical in certain applications where power supply is limited, such as MR elements used in spacecrafts [226, 229], MR sensors integrated into portable devices [96, 98, 99], and also MR sensors for the Internet-ofThings (IoT) [408, 409]. As exhibited in Figure 11(a), an MR sensor with power consumption of $0.1 \mathrm{~mW}$ was demonstrated in 1998 [242]. After more than 10 years of development, a sensitive 64-element MTJ sensor was fabricated by Liou et al. in 2011 and each MTJ element only dissipated $\sim 16 \mu \mathrm{W}$ of power [243]. The power consumption of MR sensors was then further reduced to $\sim 3 \mu \mathrm{W}$ by Yin et al. in 2014 [245]. In the same year (2014), Honeywell released two nano-powered MR sensors (SM353LT, SM351LT) in which power consumptions were as low as $\sim 510 \mathrm{nW}$ and $\sim 590 \mathrm{nW}$, respectively [244]. By fitting the historical development over the last two decades with a linear line, one can expect MR sensors with ultralow power consumption of $\sim 1 \mathrm{nW}$ (Milestone of power consumption: $\mathrm{M}_{\text {pow }}$ ) in 2022 .

Another operational parameter is the mechanical flexibility of MR sensors [64-87], which is crucial for MR sensors installed in flexible devices or for MR sensors sustaining mechanical strains. The development trend of the mechanical flexibility of MR sensor can be divided into three levels, namely, moderately flexible (fabricated on a planar substrate), highly flexible (bendable or able to be elongated), and extremely flexible (twistable) in Figure 11(b). In "Moderately flexible" level, MR sensors deposited on/in different flexible 

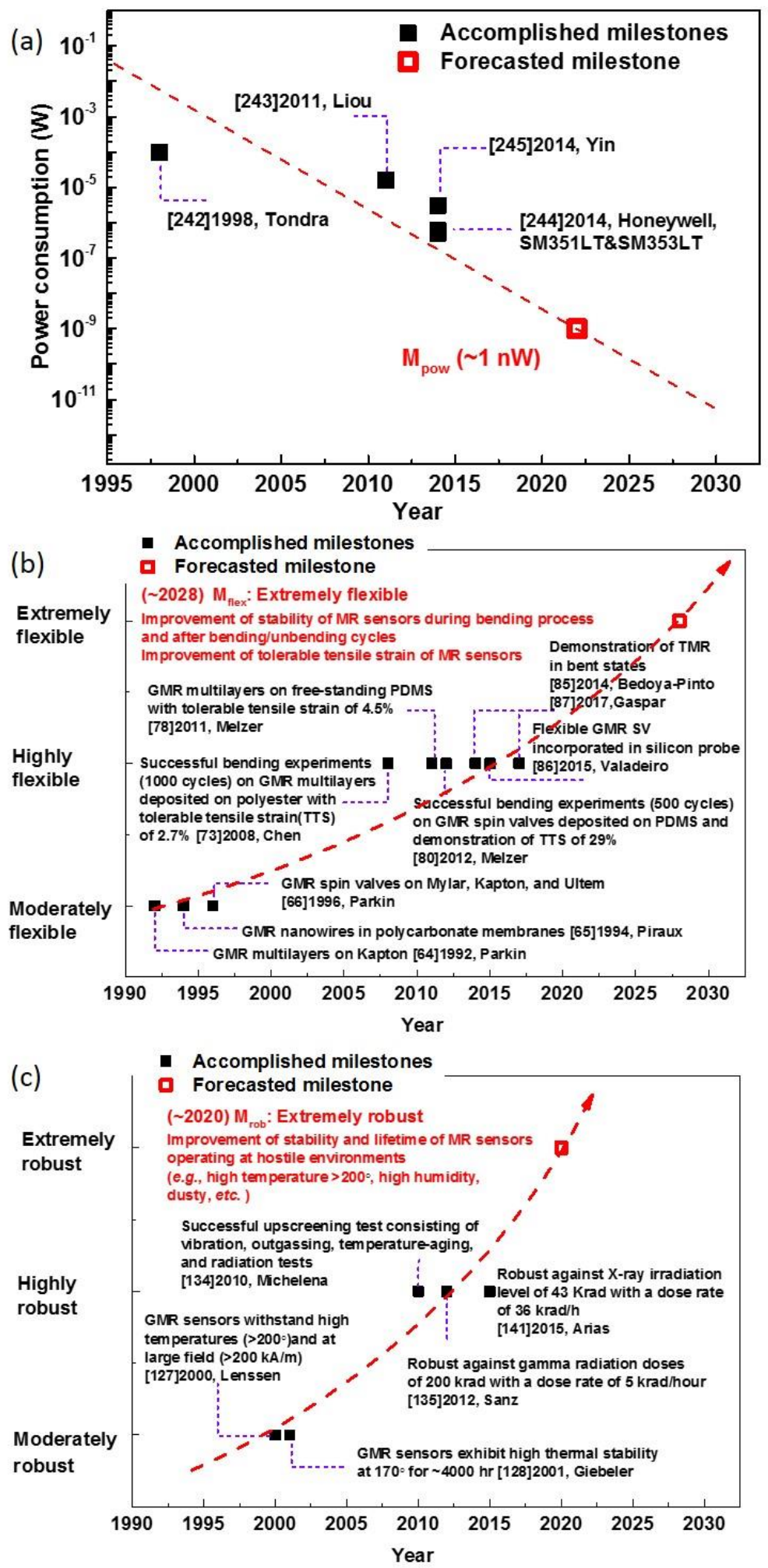

Fig. 11. Development trend of (a) power consumption, (b) mechanical flexibility, and (c) robustness of MR sensors from 1990 to 2032. PDMS represents poly(dimethylsiloxane) membranes.

materials in a planar substrate were fabricated $[64-66,68,70]$. Parkin et al. fabricated the first flexible GMR multilayer sensor on a kapton substrate in 1992 [64]. In 1994, growth of GMR nanowires in etched polycarbonate membranes were reported.
Since then, MR sensors grown on a variety of planar substrates were realized, such as mylar, kapton, ultem, polypropylene sulfide, polystyrene, and poly (2-vinyl pyridine) $[65,66,68$, 70]. After these achievements, mechanical flexibility of MR 
sensors was tested and characterized through bending and elongation in the period of 2008 to 2017 (highly flexible) [73, 78, 80, 85-87]. MR sensors with tolerable tensile strains of $2.7 \%, 4.5 \%, 29 \%$ were recorded in 2008 [73], 2011 [78], and 2012 [80], respectively. Bending experiments were performed on both multilayer (1000 bending/unbending cycles) and spinvalve (500 bending/unbending cycles) GMR sensors [73, 80]. The GMR sensors exhibited no changes in both resistance and MR ratio after bending/unbending tests. In 2014, Bedoya-Pinto et al. fabricated flexible TMR sensors on kapton substrates and obtained TMR ratio of $12 \%$ in bent state [85]. In 2015, Freitas' group incorporated MR sensors into micromachined silicon probes, which exhibited constant MR ratio and no significant changes in their noise level under a continuous tensile stress [86]. In 2017, the same group fabricated high-performance MTJ sensing devices (TMR above $150 \%$ ) on flexible polyimide substrates [87]. Under controlled mechanical stress conditions, TMR value showed subtle variation $(\sim 1 \%)$ and sensitivity changed by $7.5 \%$ when the curvature radius of the device was reduced down to $5 \mathrm{~mm}$ upon bending. These works unambiguously demonstrated the mechanical flexibility of MR sensors, elevating the mechanical flexibility level from "Moderately flexible" to "Highly flexible". From Figure 11(b), it requires around 10 years to develop MR sensors from "Moderately flexible" to "Highly flexible" and each stage lasts for around 10 years. We therefore expect that the future milestone of mechanical flexibility $\left(\mathrm{M}_{\mathrm{flex}}\right.$ : "Extremely flexible") will be reached in 2028 with further improvements on stability of flexible MR sensors and their tolerable tensile strain. In this stage, the MR sensors are expected to maintain the MR ratio even after twisting, and thus can be made into almost any shape [66, 410]. This extremely flexible performance of MR sensors will allow many future use of organic electronics for bio-applications by forming the MR sensors on organic substrate [53].

In addition to the mentioned operational parameters, the robustness of MR sensors is one of the paramount issues, especially for sensors operating in hostile environments. Similarly, the development trend of the robustness of MR sensors is summarized into three levels, namely, moderately robust (only thermal endurance), highly robust (multi-degree environment endurance such as temperature, irradiation, and vibration), and extremely robust (high endurance in multidegree environment) in Figure 11(c). In "moderately robust" level during the period of 2000 to 2001, basic tests on robustness of MR sensors were conducted on their thermal stability. In 2000, Lenssen et al. tested the thermal and magnetic stability of GMR sensors at high temperatures $\left(>200^{\circ} \mathrm{C}\right)$ and large magnetic field (>200 kA/m) [127]. In 2001, GMR sensors operating with high stability at $170^{\circ} \mathrm{C}$ for $\sim 4000 \mathrm{~h}$ were reported [128]. In "highly robust" level, the robustness of MR sensors was systematically validated in multi-degree environments. For example, the application of MR sensors was validated in aerospace by performing the up-screening tests and irradiation tests in 2010 [134]. The up-screening tests included a series of tests, such as vibration, outgassing, and temperature-aging.
In another published work in 2012, a systematic gamma irradiation test of MR sensors was carried out [135]. AMR sensors were tested to be robust against radiation doses of 200 $\mathrm{krad}$ with a dose rate of $5 \mathrm{krad} / \mathrm{h}$. In 2015, X-Ray irradiation test of TMR sensors was performed by Freitas' group under total dose level of $43 \mathrm{krad}$ with a much higher dose rate of $36 \mathrm{krad} / \mathrm{h}$ [141]. The device sensitivity exhibited a slight reduction during the irradiation and recovered afterwards. From Figure 11(c), since there has been steady progress in robustness level in the past two decades (from "Moderately robust" in 2000 to "Highly robust" in 2010), we can expect MR sensors will be demonstrated to be extremely robust (Milestone of robustness: $\mathrm{M}_{\text {rob }}$ ) by $\sim 2020$. The achievement of $\mathrm{M}_{\text {rob }}$ will enable advanced applications that critically rely on sensor robustness (e.g., MR sensor with high stability and long lifetime operating in hostile environments).

These achievements indicate that MR sensors are promising candidates for a wide range of applications where power saving, mechanical flexibility, and robustness are of significant importance.

\section{MR SENSOR APPLICATIONS AND FUTURE DIRECTIONS}

Continuous research and engineering efforts on MR sensors have remarkably improved their sensitivity, detectivity, mechanical flexibility, power consumption, and robustness as discussed in Section IV, opening up a wide range of applications [29-31, 33, 34, 37-46, 48, 50, 51, 53-55, 78, 97, 109, 120, 219-223] as shown in Figure 5. Main MR sensor applications can be categorized into five areas, including biomedical applications, flexible electronics, PS and HCI, NDEM, navigation and transportation. To shed light on the future directions of MR sensor applications, five roadmaps for these five application areas were developed. The historical data from literature analysis was fitted with the logistic growth model to obtain the fitted trend curve. The fitted curve was then further adjusted and fine-tuned based on the critical milestones for sensor parameters developed in Section IV and the consensus of the professional judgements reached during the taskforce meetings and subsequent communications. Roadmaps predicting new opportunities for MR sensor technology in different application areas were created based on these extrapolated trend curves. Speculations about new MR applications, products, and services were presented for the next 15 years and beyond.

\section{A. Biomedical applications}

Regarding MR sensor applications in the biomedical field, the detectivity of MR sensors is a paramount issue because the generated biomagnetic signals are usually rather small, ranging from nT to fT [14-46, 48-58, 222]. The roadmap is shown in Figure 12. Biomedical applications for MR sensor technology can be categorized into two scenarios $\left(\mathrm{S}_{\text {biomed }}\right)$ :

$\mathrm{S}_{\text {biomed }} 1$. MR sensors to detect magnetic signals generated from bio-functionalized nanoparticles/nanostructures 
$\mathrm{S}_{\text {biomed }} 2$. MR sensors to directly detect magnetic signals generated from human organs (e.g., brain, heart, muscles, etc.)

In $\mathrm{S}_{\text {biomed }} 1$, as MR sensor technology improved and matured after the basic technology research stage (TRL 1-2) from 1975 to 1990 , the feasibility of applying MR sensors in biomedical research was investigated during the period from 1990 to 2004 $[16,18-21,23,24,26,27]$. In 1998, the measurements of intermolecular forces between DNA-DNA, antibody-antigen, or ligand-receptor pairs were demonstrated by using GMR sensors [16]. In 2001, the detection of DNA hybridization was achieved by using GMR sensor arrays [18]. The feasibility of adopting MR sensors in biomedical applications was preliminarily proved and TRL reached 3 .

This technology was then further developed by several groups. In 2002, a group from the Instituto de Engenharia de Sistemas e Computadores and Instituto Superior Tecnico introduced a method to control the movement of nano/microsized magnetic labels and demonstrated the detection of single microspheres bonded with biomolecules [19]. In addition, AMR sensors were used to detect micro-sized nanoparticles and an AMR-based bio-sensor prototype was proposed in 2002 [21]. In 2003, the biological binding of single streptavidin functionalized magnetic microspheres on the surface of GMR sensors was detected by Graham et al. from INESC-MN (former INESC) and IST [23]. In the same year, Wang's group in Stanford successfully detected the presence of magnetic particles (Dynabead, $2.8 \mu \mathrm{m}$ in diameter) with micro-scaled spin-valve GMR sensors [60]. All these works laid the groundwork and revealed the feasibility of adopting MR sensors in biomedical research and indicated that MR sensors can be utilized to develop biomedical technology (TRL 3-4).

After 2004, further development of biomedical technology with MR sensors then proceeded and focused on detecting magnetic signals generated from biofunctionalized magnetic nanoparticles/nanostructures [29-31, 33, 34, 37-39, 41-46, 48, 51, 53-55, 191, 222].

In the period of 2005 to 2008, the detection of biofunctionalized nanoparticles/nanostructures with MR sensors was demonstrated in both in-vitro and in-vivo conditions [29$31,33,34]$. In 2005, cystic fibrosis related DNAs were successfully detected with spin-valve GMR sensors by using an AC magnetic field focusing technique [29, 30]. Grancharov et al. successfully detected protein-functionalized and DNAfunctionalized monodisperse nanoparticles with a TMR biosensor [31]. These results suggested that MR bio-sensors were validated in laboratory environment and TRL 5 was achieved.

Since then, bio-sensing applications with MR sensors were developed in relevant environments [35-37, 39, 42, 43, 48, 51, 55]. At the $29^{\text {th }}$ IEEE Engineering in Medicine and Biology Society conference in 2007, an AMR-based biomagnetic prototype was demonstrated to evaluate the gastric activity contractions and in-vivo tests were performed [35, 36]. In 2008, a portable bio-sensing prototype was developed and the detection of magnetic nanoparticles was demonstrated [37]. In the same year, Wang's group developed a GMR-based biochip

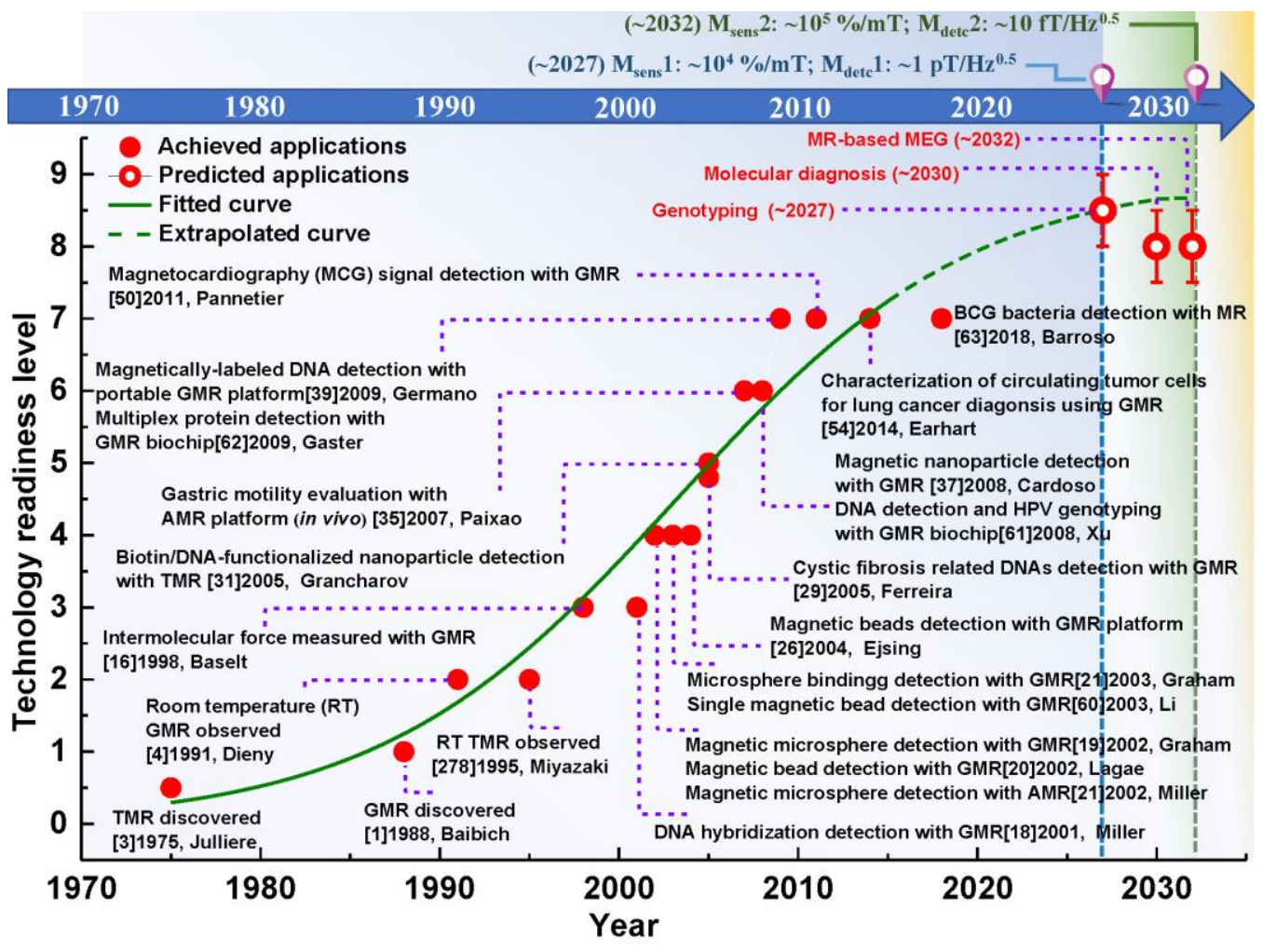

Fig. 12. Roadmap for MR sensors in biomedical applications from 1970 to 2032. 
for DNA detection and human papillomavirus (HPV) genotyping [61]. Their work also showed real-time signal responses of multiple DNA fragments, which demonstrated the multiplex detection capability of the GMR-based biochip. These works revealed that MR-based bio-sensing prototypes were tested and implemented in practical environment and TRL 6 was reached.

After 2008, bio-sensing chips/systems with MR sensors were developed and thus MR sensor-based biomedical technology was elevated to a higher level. In 2009, a portable GMR platform was demonstrated to detect magnetically-labelled DNA by Germano et al. [39]. Furthermore, Wang's group developed a multiplex GMR-based bio-sensing platform for protein detection in blood and cell lysates [62]. The developed platform exhibited an extensive linear dynamic range over six orders of magnitude and a protein detecting resolution down to attomolar level. In 2014, the detection and characterization of circulating tumor cells (CTCs) were conducted with a GMRbased biochip and CTCs were detected in the blood samples from lung cancer patients [54]. In 2018, the detection of Bacillus Calmette-Guérin bacteria was also carried out with an MR-based bio-sensing platform for tuberculosis diagnosis [63]. These works elevated the laboratory achievements of MR biosensor technology to the clinical/near-clinical level (TRL 7).

Compared to MR sensor applications in $S_{\text {biomed }} 1$, the requirements of MR detectivity is much higher in $S_{\text {biomed }} 2$, which is attributed to the fact that the generated magnetic signals from human organs are merely in the range of pT (e.g., magnetic field produced by heart) to fT (e.g., magnetic field produced by brain) [14]. For the biomagnetic signals produced from human organs, two most-investigated signals are generated from the heart and brain. These signals contain valuable information and lead to two application areas, magnetocardiography (MCG) $\left[\begin{array}{lll}17, & 22, & 50\end{array}\right]$ and magnetoencephalography (MEG) [14], respectively. Seven years after the detectivity of pT range was reached in 2004 [28], MCG biomagnetic signals from healthy volunteers were recorded and a magnetocardiography MCG signal distribution was mapped with a highly sensitive (pT) GMR sensor in 2011 [50]. These technology demonstrations indicated that biosensing subsystems/systems with MR sensors were validated in operational environments, and TRL 7 was achieved.

To predict and outline the future biomedical applications, the above historical biomedical developments summarized from the published literature were analyzed using the logistic growth model and the extrapolated trend curve was established (Figure 12). Adjustment of the curve was then performed based on the critical milestones for sensitivity and detectivity derived in Sections IV(A) and IV(B) and the professional assessment consensed by the roadmap taskforce. Likely biomedical applications with MR sensors were then predicted and their TRL levels were estimated.

Synthesis of DNA-functionalized or even DNA-basesfunctionalized nanoparticles will possibly enable commercialized genotyping applications [49] with MR sensor technologies. With the achievement of $\mathrm{M}_{\text {sens }} 1\left(\sim 10^{4} \% / \mathrm{mT}\right)$ and $\mathrm{M}_{\text {detc }} 1\left(\sim 1 \mathrm{pT} / \mathrm{Hz}^{0.5}\right)$ in $\sim 2027$, MR sensors can be used to accurately detect the real-time magnetic signals from magnetically-labeled DNA fragments or entities. After improving the multiplexing features [41, 45, 61] and localized detection ability of MR sensors [34], we expect that commercialized genotyping products with MR sensors will be released and the corresponding TRL of level 8-9 will be achieved.

The development of genotyping applications with MR sensors will promisingly facilitate the diagnosis and treatment of genetic diseases. Continuous efforts on synthesis of various bio-functionalized magnetic nanoparticles or nanostructures $[23,31,40]$ will stimulate the application of highly-sensitive MR sensors in molecular diagnosis [15, 25]. However, the MRbased molecular diagnosis systems are required to be validated and their commercialization requires Food and Drug Administration (FDA) clearance from the government of the targeting market. We therefore expect that MR-based molecular diagnosis products or services will be commercially available a few years later than genotyping and its maturity will reach a slightly lower TRL of level $\sim 8$ in 2030 . This accomplishment can promisingly offer personalized diagnosis and possibly lead to optimized therapies for individual patients.

On the other hand, a more challenging category of application, MR-sensor-based MEG requires fT range detectivity and therefore will be developed after the achievement of $\mathrm{M}_{\text {sens }} 1\left(\sim 10^{4} \% / \mathrm{mT}\right)$ and $\mathrm{M}_{\text {detc }} 1\left(\sim 1 \mathrm{pT} / \mathrm{Hz}^{0.5}\right)$ in 2027. Through further improvement of sensitivity and detectivity towards $\mathrm{M}_{\text {sens }} 2\left(\sim 10^{5} \% / \mathrm{mT}\right)$ and $\mathrm{M}_{\text {detc }} 2(\sim 10$ $\left.\mathrm{fT} / \mathrm{Hz}^{0.5}\right)$ respectively, one can expect the implementation of MR-sensor-based MEG applications (TRL 8) with elaboration on clinical level around or after 2032.

Apart from MR sensing elements, the other key factors such as magnetic labels, surface chemistry, microfluidic systems and electronics setup are critical to achieve high-performance, automated, portable point-of-care bioanalytical assays [411]. The size of the MR sensing element and the bio-molecule binding capacity of the magnetic particles need to be carefully designed [9]. A reliable biochip platform needs a fine control of the surface chemistry in order to achieve immobilization efficiency and specificity and avoid corrosive effect. A microfluidic system is required to establish mechanism for sample delivery protocol and controlled washing [411]. Last but not the least, the system miniaturization of signal processing and system automation will be implemented with electronics microsystems for building point-of-care devices [412, 413].

\section{B. Mechanically flexible electronics}

Flexible electronic devices have gained increasing interest due to the promising potential applications offered by their pliable surface geometries [78, 81, 83, 85]. MR-based devices have been implemented on various types of flexible substrates, such as stretchable and deformable polymeric materials $[64,70$, 
$75,78,81,85]$, and even papers [79, 83]. This roadmap is shown in Figure 13.

The flexible MR sensors are required to be robust against mechanical bending or stretching and withstand many cycles of deformations without the degradation of sensing performance. The emergence and growth of the flexible MR sensor technology took place in the period of 1992-2007 [64-72]. In 1992, Parkin et al. investigated the GMR effect in $\mathrm{Co} / \mathrm{Cu}$ multilayers deposited on a Kapton polyimide substrate by magnetron sputtering [64]. In 1994, growth of GMR nanowires in etched polycarbonate membranes were reported by Piraux et al. [65]. Two years later (1996), Parkin successfully fabricated spin-valve GMR sensors on other flexible organic films (mylar, a transparent film, and ultem polyimide) [66]. These works built the foundation and proved the feasibility of manufacturing flexible MR sensors, pushing the TRL of the flexible MR sensor technology towards level 3 .

This technology was then further developed by several groups. In 2002, Yan et al. deposited GMR multilayers on flexible polypyrrole films [68]. The mechanical flexibility of the prepared GMR film was tested by cutting it into various shapes. In 2006, Uhrmann et al. reported the mechanical flexibility of GMR spin valves grown on polyimide substrates and the sensors were elastic up to an elongation of 3\% [70]. These studies further proved the feasibility of flexible MR sensor technology and TRL 4 was reached.
After 2006, the mechanical flexibility of MR sensors was tested through the bending and strain experiments $[73,78,80$, $85]$. In 2008, tensile strain measurement was carried out on the GMR sensors on polyester substrates and the stress was applied to the GMR sensors by performing in-plane elongation [73]. The sensors exhibited great stability and withstood 1000 bending/unbending cycles with no degradation of GMR ratio. In 2011, multilayer GMR sensors on free-standing polydimethylsiloxane membranes revealed a high GMR of $50 \%$ and the GMR effect was preserved with tensile strain up to $4.5 \%$ [78]. These works demonstrated the mechanical flexibility of MR sensors and pushed the TRL towards level $\sim 5$.

The mechanical flexibility of MR sensor was then further enhanced. In 2012, the tolerable tensile strain as high as $29 \%$ was achieved by depositing spin valves on pre-stretched and pre-wrinkled polydimethylsiloxane substrates [80]. In 2014, Bedoya-Pinto et al. successfully deposited TMR sensors on

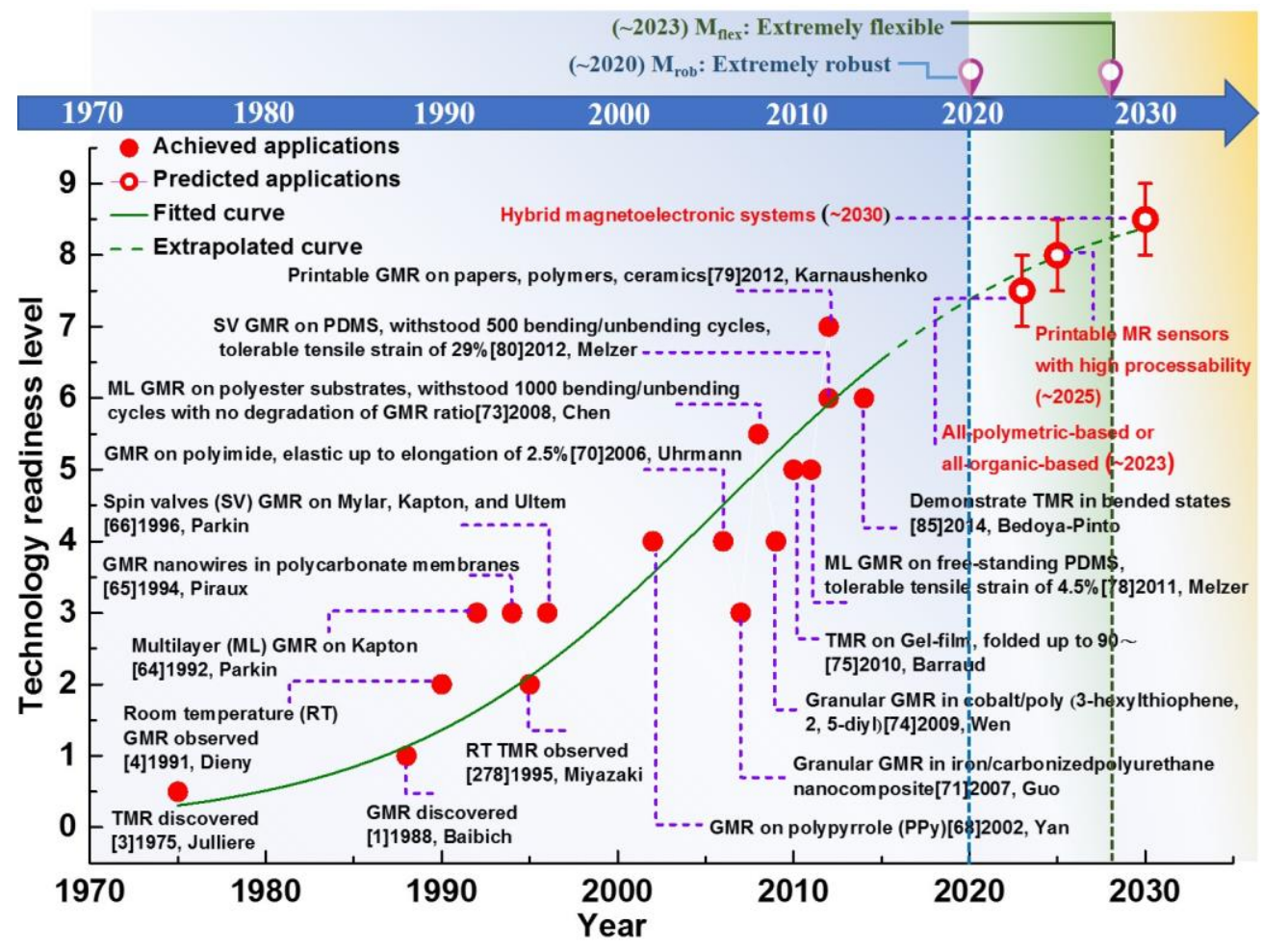

Fig. 13. Roadmap for MR sensor applications in flexible electronics from 1970 to 2032. 


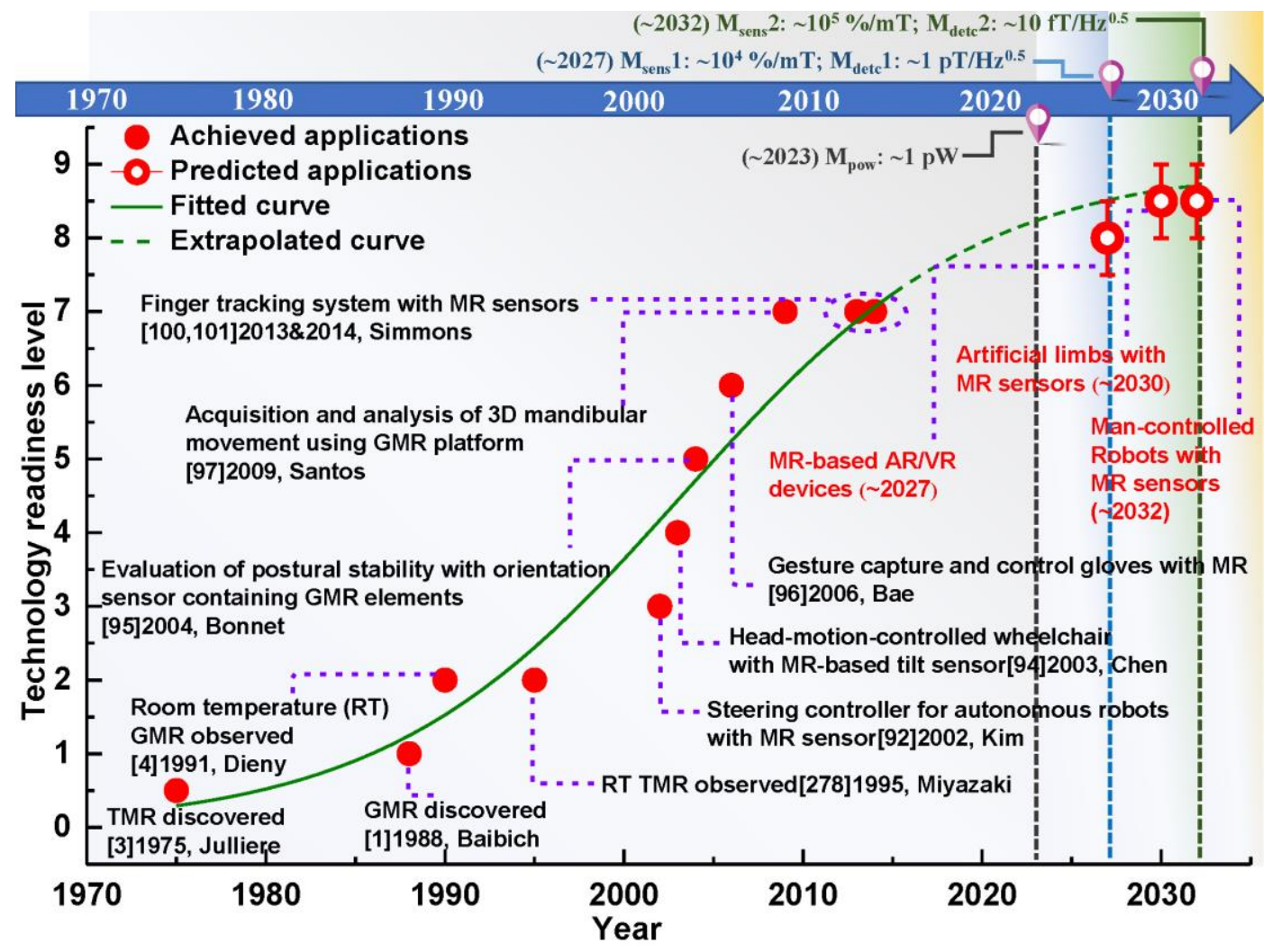

Fig. 14. Roadmap for MR sensor applications in PS and HCI from 1970 to 2032.

kapton substrates and demonstrated the preservation of TMR effect in bent states [85]. Also, flexible MR sensors prepared with printable magneto-sensitive inks were reported by Karnaushenko et al. [79]. The printable MR inks were prepared by a process including magnetron sputtering, rinsing, ball milling, and mixing. The prepared inks were then painted on various substrates (e.g., papers, polymers, and ceramics) and the fabricated sensors with GMR response up to $8 \%$ were demonstrated. This fabricated GMR sensor was integrated into a paper-based electronic circuit and acted as a magnetic switch of the whole circuit, which confirmed the functionality of flexible sensing systems/subsystems with MR sensors. These works revealed that the mechanical flexibility of MR sensors was validated in practical environments and TRL reached level 6 and approached early stage of level 7.

The enhancement of mechanical flexibility will enable the applications of MR sensors in wearable and portable electronics. Most of the reported flexible MR sensors were composed of a flexible polymeric substrate and a conventional MR multilayer structure [53, 64, 66, 68, 70-73, 75, 78, 80, 84, 85]. Although the polymeric substrate was robust against mechanical deformations, the MR response of the multilayer tended to degrade after many bending cycles [73], which essentially limited its sensing performance. To resolve this issue, all-polymeric-based (APB) or all-organic-based (AOB) MR devices are required to be developed, which is a promising pathway toward highly deformable and bendable MR sensors.
An important step forward for the APB or AOB MR devices was the demonstration of MR effect in an organic spin valve where the organic $\mathrm{V}[\mathrm{TCNE}]_{\mathrm{x}}(\mathrm{x} \sim 2$, TCNE: tetracyanoethylene) served as ferromagnetic layers and the rubrene $\left(\mathrm{C}_{42} \mathrm{H}_{28}\right)$ was used as the insulating barrier [77]. After the achievement of $\mathrm{M}_{\text {rob }}$ (extremely robust) in 2020 and the development of sensor mechanical flexibility towards $\mathbf{M}_{\text {flex }}$ (extremely flexible) in $\sim 2028$, one can expect the realization of APB or AOB MR system (TRL 7-8) in 2023 with higher mechanical flexibility as well as better robustness through performing necessary deformation and bending evaluations.

The implementation of APB or AOB MR sensors will lead to the achievement of fabricating MR sensors with higher mechanical flexibility as well as better robustness, promoting the application of MR sensors in wearable, portable, and printable electronics. Particularly, the printable MR sensors will revolutionize the field of magnetoelectronics offering low-cost and large-scale production in manufacturing processes. Through research efforts on the synthesis and optimization of MR inks, paints, and pastes, we expect that the printable MR sensors with high processability (TRL 8) can be accomplished in a short period (in 2025 ).

Then hybrid magnetoelectronic devices can be developed by integrating printed MR sensors in a purpose-designed electronic circuit (e.g., authorization, monitoring, data recording, etc.). The integrated MR sensor can serve as a magnetic-information acquisition element or a magnetically-manipulable option in the 
hybrid magnetoelectronic devices. However, the implementation of actual hybrid magnetoelectronic systems (TRL-9) will be expected within five years (in 2030) after the demonstration of the high processability of printable MR sensors. The development of printable MR sensors can promisingly reduce the fabrication cost, weight, and physical dimension of MR sensors by replacing conventional substrates (Si) with standard printing materials (paper, polymer, ceramics), promoting the high-volume production of printable magnetoelectronics.

\section{Position sensing (PS) and human-computer interaction (HCI)}

Owing to the high sensitivity, low power consumption and small physical dimension, MR sensors have been considered as promising magnetic sensors embedded in PS applications [8891] and HCI systems [94-101, 414]. This roadmap is shown in Figure 14.

In PS applications, MR-based linear and angular sensors are used to acquire incremental or absolute scale data from magnetic linear rulers, code wheels, and human body [88-91, 94, 96, 97, 100, 101]. Through software development and integration of computer interface, the obtained information can be processed and further utilized in HCI implementations.

In the period of 2002 to 2003, the feasibility of integrating MR sensors into PS and HCI was investigated [92-94]. In 2002, an MR-sensor-based steering controller for outdoor mobile robot was designed [92]. A computer simulation was performed to verify the performance of the controller. In 2003, Chen et al. proposed a head-motion-controlled wheelchair with an MRbased tilt sensor integrated into the headgear [94]. The comfortability and safety of the developed wheelchair were tested and verified. Basic biomechanical motions were captured and processed in these works, which proved the feasibility of integrating $\mathrm{MR}$ sensors into $\mathrm{PS}$ and $\mathrm{HCI}$ and raised the corresponding TRL to $3-4$.

This technology was further investigated and the acquisition and analysis of more complicated biomechanical motions and postures were carried out [95-101, 414]. In 2004, Bonnet et al. introduced a novel method to evaluate the postural stability with an orientation sensor containing GMR magnetometers and accelerometers [95]. By virtue of the high sensitivity of the orientation sensor, subtle postural variations were captured and could be utilized in clinical balance assessments. In 2006, Bae et al. were able to track the wrist gestures and control the movements of the robot with GMR-based wearable gloves [96]. These works demonstrated the operation of HCI prototypes with MR sensors and boosted the TRL to 5-6.

The HCI systems/subsystems were then developed and the TRL was elevated to a higher level. In 2009, the acquisition of three-dimensional mandibular movements was realized by using a GMR-based device by Santos et al. [97]. A computer application was developed to analyze the movements and generate diagnosis reports. In the period of 2013 to 2014, a 3 degree-of-freedom (DOF) finger tracking system was demonstrated by using a commercially available 3-axis MR sensor [100, 101]. Both finger joint position and finger movement configurations (stationary joint, flexing joint, etc.)

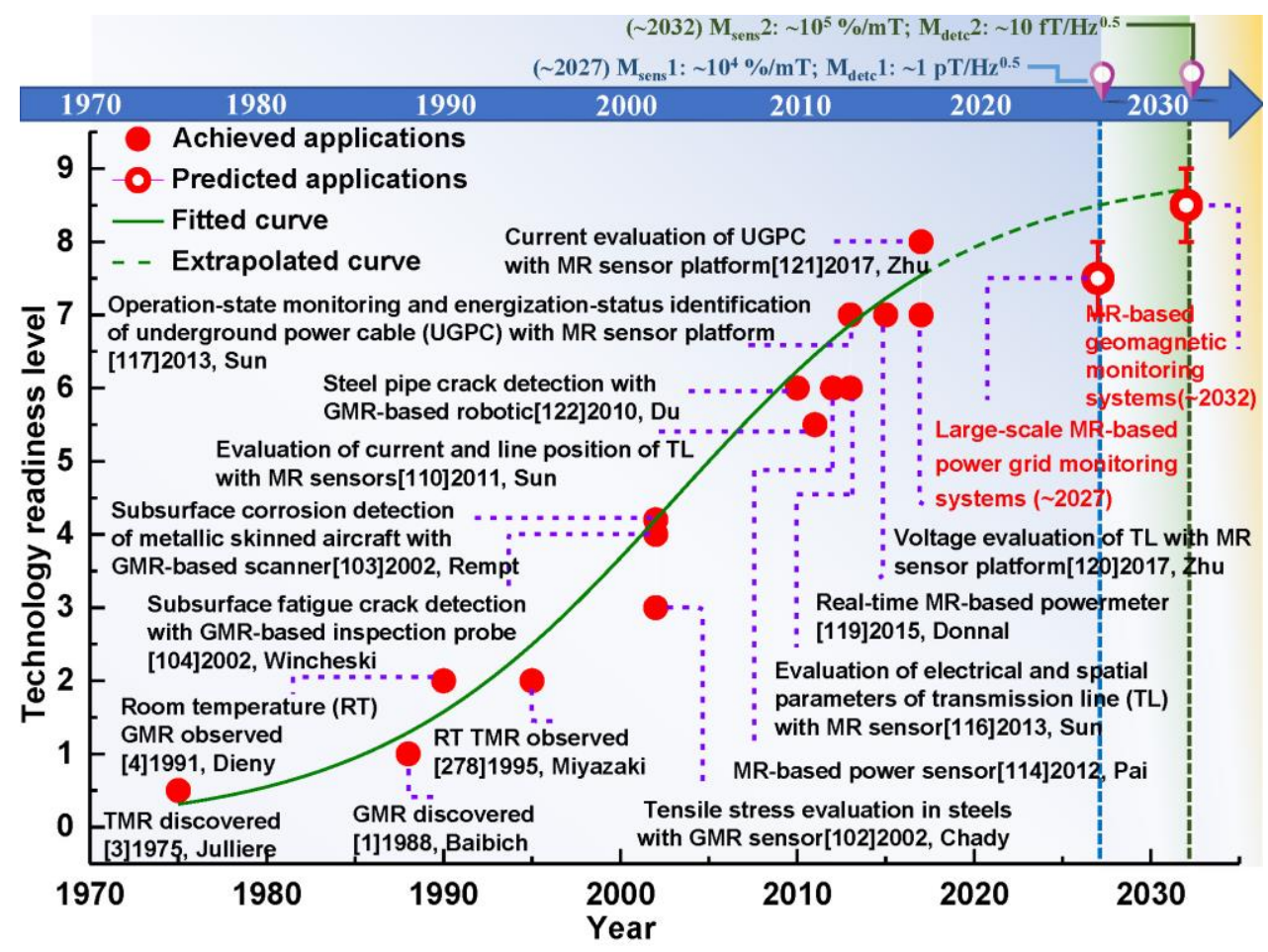

Fig. 15. Roadmap for MR sensor applications in NDEM from 1970 to 2032. 
were captured and evaluated. These works validated the operational performance of the MR-sensor-based HCI systems/subsystems and suggested that the TRL entered level 7.

Based on past developments and professional consensus of the roadmap taskforce members, the future potential MR-based HCI applications were predicted. As demonstrated in the reported HCI systems with MR sensor description, biomechanical movements of various body parts can be effectively captured and recorded by processing and analyzing the acquired magnetic data. This type of biomechanical data will likely be used in the field of AR and VR. With the achievement of enhanced sensitivity $\left(\mathrm{M}_{\mathrm{sens}} 1, \sim 10^{4} \% / \mathrm{mT}\right)$ and detectivity $\left(\mathrm{M}_{\mathrm{detc}} 1, \sim 1 \mathrm{pT} / \mathrm{Hz}^{0.5}\right)$ in $\sim 2027$, one can expect that AR/VR devices integrated with high-performance MR sensors (TRL 8) will be available.

Commonly-used joysticks will then be replaced by wearable MR-based controllers to realize uncumbersome HCI interfaces. MR sensors can also be integrated into artificial limbs of disabilities and the obtained biomechanical signals can be processed to assist their desired movements.

Further improvement of sensitivity and detectivity will enable accurate detection of biomechanical signals and reduction of power consumption $\left(\mathrm{M}_{\text {pow }}, \sim 1 \mathrm{pW}\right)$ will extend the lifetime of the artificial limbs with MR sensors, which will push forward its maturity level to 8-9 in around 2028. Furthermore, the implementation of MR-based man-controlled robots will be possibly realized by collecting and processing all the biomechanical movements. However, such technology will require a tremendous amount of tests and assessments and further improvement of MR sensor performance $\left(\mathrm{M}_{\text {sens }} 2, \sim 10^{5}\right.$ $\left.\% / \mathrm{mT} ; \mathrm{M}_{\mathrm{detc} 2}, \sim 10 \mathrm{fT} / \mathrm{Hz}^{0.5}\right)$. We therefore estimate that the full maturity (i.e., TRL 8-9) of the MR-based man-controlled robots will be accomplished around 2032 .

\section{Non-destructive evaluation and monitoring (NDEM)}

Compared to destructive sensing devices, NDEM with MR sensors can be easily installed and accessed by end users, enabling effective acquisition of magnetic or magnetic-related information from the subsystems/systems under monitoring [102-104, 107, 108, 110, 111, 113, 114, 116-122]. This roadmap is shown in Figure 15.

The feasibility of utilizing MR sensors in NDEM was first tested by several groups in 2002. The MR-sensor-based NDEM of subsurface mechanical and chemical damages in metallic or magnetic components was introduced, especially, to investigate the components used in high-standard products (e.g., aircrafts) [102-104]. A GMR-based inspection probe was developed to detect the subsurface fatigue cracks and holes under airframe fasteners [104]. The functionality of the developed probe was studied by both finite-element-method simulation and experiment. In the same year, a GMR-based gradiometer was introduced to measure the tensile stress of the SS400 steels [102]. Ray Rempt from the Boeing company also proposed an 8 -element MR scanner for inspecting the subsurface corrosion of the airframe [103]. The stress damages in the steels were evaluated and visualized by interpreting the sensor data with a signal processing algorithm. These results suggested that the feasibility of NDEM technique with MR

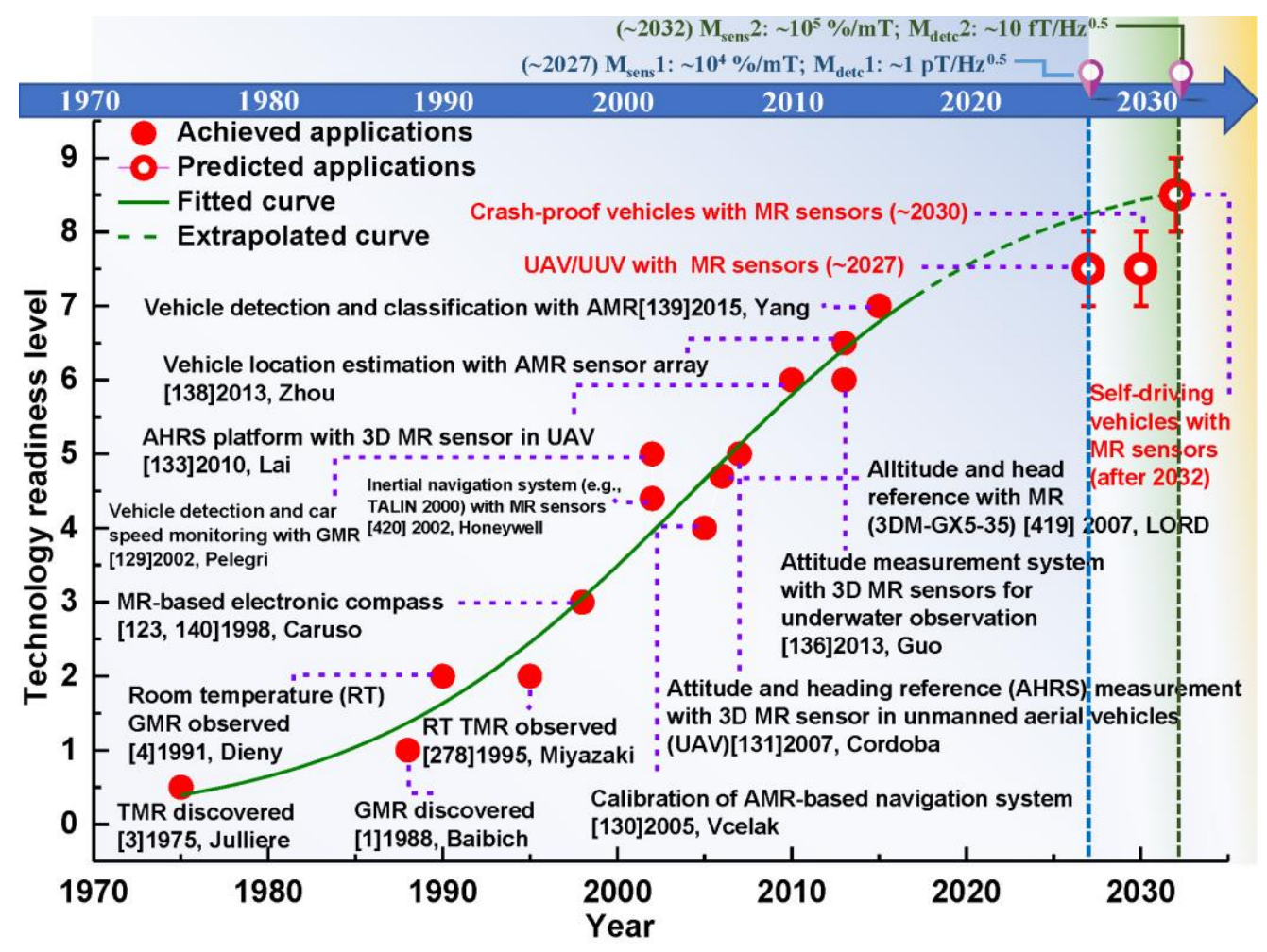

Fig. 16. Roadmap for MR sensor applications in navigation and transportation from 1970 to 2032. 
components/breadboards was validated in practical conditions. The maturity of NDEM with MR sensors reached TRL 3-4.

Another promising application of the non-destructive MR sensors is the evaluation and monitoring of the power grids. Abundant studies demonstrated the feasibility of using MR sensors for monitoring both the high-voltage overhead transmission lines and underground power cables [106, 110, $111,113,114,116-121]$. In 2011, a proof-of-concept laboratory setup was constructed to determine the phase current and line position of transmission lines by Sun et al. [110]. In 2012, Pai et al. introduced an MR-based power meter to measure nearfield voltage and current waveforms of a power cord [114]. Accuracy of power measurement better than 5\% was accomplished. These works demonstrated the operation performance of NDEM prototype with MR sensors and indicated the achievement of TRL 5-6.

Further studies were performed to establish MR-sensorbased NDEM systems/subsystems. Pong's group proposed and developed several novel MR-based platforms to monitor the loading voltages and currents of power lines [111, 116-118, 120, 121]. The MR-based monitoring platforms were able to characterize the fault location [111] and operation state of the power lines by extracting the loading current data [116]. Utilizing the capacitive-coupling between the power lines and induction bars, the voltages of the power lines were accurately evaluated and the ability of high-frequency transient measurement was demonstrated [120]. The phase current of the power line was reconstructed by analyzing the magnetic field from the power lines. The feasibility and accuracy of the proposed method were verified by a scaled laboratory platform and then validated by performing an on-site experiment in a substation [121]. The MR-assisted voltage monitoring system was validated with a scaled testbed [234]. These achievements demonstrated that the validation of MR-sensor-based NDEM systems in practical environment and marked the maturity of NDEM technology with MR sensors (TRL 7-8).

Continuous efforts on improving sensing performance of MR sensors will promote the development of MR-based NDEM systems. The maturity of this application will enable large-scale evaluation of key parameters of power grids, such as current [106, 113, 114, 116], voltage [114, 119, 120], phase [110, 116, 117], power flow [114, 119], power quality [119], load [117, 119], transmission and distribution line conditions $[111,116,117,120]$. By analyzing and processing the power grid parameters, the real-time state of power grids can be evaluated, enabling the prompt determination and response of power faults or abnormal conditions in a wide area. After the achievement of $\mathrm{M}_{\text {sens }} 1\left(\sim 10^{4} \% / \mathrm{mT}\right)$ and $\mathrm{M}_{\text {detc }} 1\left(\sim 1 \mathrm{pT} / \mathrm{Hz}^{0.5}\right)$ in 2027, the implementation of the large-scale power grid monitoring systems with MR sensors (TRL 7-8) will be expected. The full establishment of these systems (TRL 8-9) will require a large quantity of supporting facilities (e.g., energy harvesting for outdoor sensors $[415,416]$, and a common time source for synchronized measurements [417, 418]), and therefore will be realized in a long-term period (after 2027).
With the further improvement of MR sensor sensitivity and detectivity to $\mathrm{M}_{\text {sens }} 2\left(\sim 10^{5} \% / \mathrm{mT}\right)$ and $\mathrm{M}_{\text {detc }} 2\left(\sim 10 \mathrm{fT} / \mathrm{Hz}^{0.5}\right)$ in $\sim 2032$, another promising field of application is a large-scale geomagnetic monitoring system, which will be utilized to monitor subtle geomagnetic disturbances related to some geomagnetic hazards, such as seismic activities [109]. MR sensors can be installed on a large seismically-active zone to monitor abnormal geomagnetic changes that are associated with seismic activities. With the assistance of a reference permanent magnet, MR sensors can also be used as displacement sensors to detect the abnormal disturbances related to foreshock patterns or plate dynamics [109]. However, the implementation of a reliable geomagnetic monitoring system with MR sensors (TRL 8-9) requires a long-term investigation of geomagnetism and cooperation between geological and magnetic societies, which will take more time to progress and will be realized around 2032 .

\section{E. Navigation and transportation}

MR-based magnetometers have been widely used in navigation and transportation systems as well [123-126, 129133, 136-139]. This roadmap is shown in Figure 16.

In the period of 1997 to 2005, the feasibility of applying MR sensors in navigation and transportation was investigated. In 1997, MR sensors provided a solid-state solution for building compass navigation systems for their high sensitivity, good repeatability and small size [123]. In 1998, an electronic compass with MR sensor was introduced [140]. The compass reading was tilt compensated and the disturbance from nearby ferrous materials was corrected. In 2005, an AMR-based navigation system was proposed [130]. With calibration of sensor's triplet deviation, the introduced navigation system provided information about actual azimuth, roll and pitch with improved accuracy. In 2005, a dead-reckoning navigation system was developed for pedestrians with an array of accelerometers and MR sensors. MR sensors became capable of collecting more informative data by virtue of the development and commercialization of 3-axis/3D MR-based magnetometers [131, 133, 136]. Commercial dead-reckoning and inertial navigation systems using MR sensors have also been developed. For example, the Lord Sensing introduced attitude and head reference systems (e.g. Lord MicroStrain 3DM-GX5-35) with MR sensors to provide attitude and navigation solutions [419]. The Honeywell introduced inertial navigation system (e.g. TALIN 2000) with MR sensors to provide navigation, pointing and weapon stabilization [420]. All these works proved the feasibility of applying MR sensors in the fields of navigation and transportation (TRL 3-4).

The technology was further developed and demonstrated from 2007 to 2010. In 2007, by integrating the 3-axis MR sensor with accelerometers and gyroscopes, a real-time attitude and heading reference system (AHRS) was reported by Cordoba et al. [131]. The constructed system was equipped in unmanned aerial vehicles (UAVs) and accurate attitude angle 
measurements were performed for the UAVs operating in both accelerated and non-accelerated conditions. To validate the AHRS in various dynamic conditions, Lai et al. designed and constructed a 3-axis rotating platform in 2010 [133], which was able to simulate dynamic conditions in the operation of different unmanned vehicles (unmanned underwater vehicles (UUVs), UAVs, self-driving vehicles). Another promising application of MR-based magnetometers is the vehicle detection and monitoring [129, 132, 138, 139], which makes use of the local magnetic field disturbance caused by moving vehicles. In 2002, a GMR-based vehicle detection and monitoring module was introduced [129]. The local magnetic field disturbance was successfully detected and the speed of the car was measured on site. These works demonstrated the implementation of MR sensors in navigation and transportation systems in relevant conditions and the accomplishment of TRL 5-6.

With the enhancement of the sensing ability of MR sensors, the functionalization and performance of the MR-based vehicle detection systems were remarkably improved [137-139]. In 2013, Zhou et al. reported the real-time location estimation of vehicles by utilizing an AMR array [138]. In 2015, the classification of various types of vehicles was achieved by analyzing the characteristics of the detected field disturbance signals [139]. These works demonstrated the possibility of achieving high-level autonomous vehicles with MR sensors, vehicles, which marked the later stage of TRL 6 for navigation and transportation systems with MR sensor technology.

Considering that the AHRS with MR sensors has already been validated in several operating conditions [131], one can expect the integration of AHRS with MR sensors (TRL 7-8) into UUVs and UAVs by $\sim 2027$ with the achievement of $\mathrm{M}_{\text {sens }} 1$ $\left(\sim 10^{4} \% \mathrm{mT}\right), \quad \mathrm{M}_{\text {detc }} 1 \quad\left(\sim 1 \quad \mathrm{pT} / \mathrm{Hz}^{0.5}\right)$. However, the implementation of crash-proof and self-driving vehicles with MR sensors would be much more difficult. MR sensors equipped in these vehicles are required to possess ultra-high sensing performance. The detected magnetic disturbance from all the surrounding vehicles and objects are required to be considered and analysed to avoid possible risks. Therefore, one can expect that the realization of crash-proof and self-driving vehicles with MR sensors (TRL 7-9) around or after 2032 with the achievement of $\mathrm{M}_{\text {sens }} 2\left(\sim 10^{5} \% / \mathrm{mT}\right), \mathrm{M}_{\text {detc }} 2\left(\sim 10 \mathrm{fT} / \mathrm{Hz}^{0.5}\right)$. Since the complexity of crash-proof vehicles is lower and technologically less complicated than that of self-driving vehicles, the authors believe that the crash-proof vehicles with MR sensors will be implemented a few years earlier than selfdriving vehicles in $\sim 2030$.

\section{OUTLOOK AND PERSPECTIVES}

The field of MR sensors is now rapidly evolving from science to technology. The proliferation of MR devices with

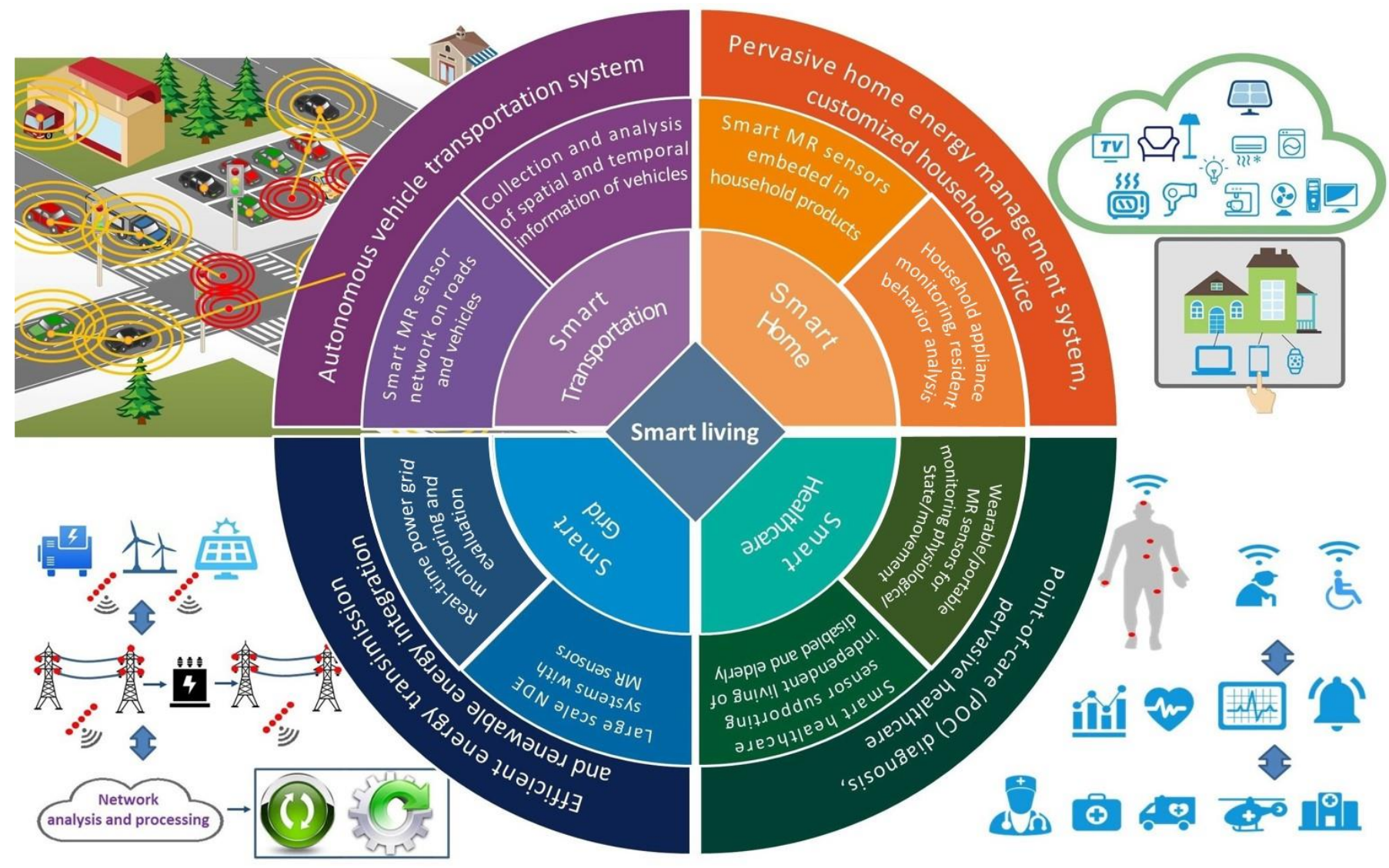

Fig. 17. Contribution and impact of MR sensor technology in the concept of smart living, including smart home, smart healthcare, smart grid, and smart such as UUVs, UAVs, crash-proof vehicles, and self-driving high operational and sensing performance is opening up a 
variety of applications based on MR technologies, such as biomedical applications, flexible electronics, PS and HCI, NDEM, and navigation and transportation. The widespread utilization of MR sensors will also offer more data and information (magnetic or magnetic-related) to the Internet of Things (IoT) [421-424], enriching and upgrading the context of smart living [425-428], such as smart home [427, 429-431], smart healthcare [425, 432-434], smart grid [105-108, 118], and smart transportation [435-438], as shown in Figure 17. One of the key supporting features of smart living is the acquisition and utilization of sufficient data and information from the "Things", which requires a large amount of networked sensors for information collection and processing [430]. Therefore, the robust MR sensors with low cost, low power consumption, small physical dimension, and superb sensing performance can be excellent candidates as networked sensors in each aspect of smart living.

A smart home is a residence equipped with sensor and communication technologies that monitor the household appliances/resident behavior and provide proactive services [425, 433]. Pervasive MR sensors can be embedded in household products, monitoring the states (e.g., on, off, standby) of household products [119]. The evaluated data can also be stored in the cloud and accessible to the residents on their smartphones, personal computers, and wearable devices. The wasteful usage of each household appliance can then be identified and avoided via adaptive control or remote control by residents. With the integration of IoT platform, a pervasive home energy management system will be developed and implemented. Furthermore, the acquired usage data of household products and residents' behavior can be analyzed and used to learn the life pattern of the resident. Customized household services (e.g., personalized household appliance automation) can therefore be delivered to the residents.

MR devices can also be used as smart-healthcare sensors to support independent living of the disabled and elderly, as well as to relieve the workload from family caregivers. Real-time physiological state or movement will be monitored with wearable/portable MR sensors [94-97, 100, 101]. Abnormal situations will be immediately alerted so that necessary assistance can be provided in time. With the development of MR-based MCG or MEG sensors [50], they can be attached on the bodies of patients with cardiac or encephalic diseases. Timely warning can be sent to the corresponding server when a cardiac or encephalic event is detected. Medical assistances and actions can then be taken by doctors and therapists. Also, lowcost, small-size, and highly wearable/portable MR biomedical sensors can be integrated into point-of-care (POC) devices [51], which can be widely distributed in hospitals, homes, and in outdoor areas. Immediate clinical services can be delivered to patients when diagnosis is completed using these POC devices. With the help of the POC technology and IoT platform, patients' past and present healthcare data will be monitored and recorded. These healthcare data will be accessible to clinicians or authorized entities. Based on the analysis and evaluation of the data, healthcare products and services can be provided in time whenever/wherever they are needed, facilitating the implementation of pervasive healthcare.

Regarding the smart grid, MR sensors can be deployed in large-scale for monitoring transmission and distribution networks. MR sensors or sensor arrays are used to monitor the real-time power grid parameters, such as current $[106,113,114$, 116], voltage [114, 119, 120], phase [110, 116, 117], power flow [114, 119], power quality [119], load [117, 119], transmission and distribution line conditions [111, 116, 117, 120]. Power grid abnormal conditions (e.g., fault, sagging, overload, and imbalance) can be evaluated and pinpointed based on analysis of measured power grid parameters $[111,116$, 117]. Necessary actions can then be performed by operation staff and predictive decisions can be made for ensuring efficient and reliable transmission and distribution of power in smart cities. The establishment of the large-scale MR-based NDEM power-grid monitoring system will provide more dynamic and pervasive monitoring information. This is critical for systematic evaluation of the existing power grid system and makes the integration of renewable energy possible.

For the smart transportation aspect, smart sensor networks with a large amount of MR sensors can be deployed on roads and vehicles and integrated into a wireless sensor network. The spatial and temporal distribution of vehicles correlates with magnetic field and can be collected by MR sensors, because a vehicle induces perturbation in the local Earth's magnetic field as it passes by a sensor $[129,138,139]$. As such, dynamic traffic information including vehicle speed [129], vehicle location [138], occupancy rate [129, 139], and traffic flow volume [129, 139] can be obtained and processed by the server. The traffic data can then be analyzed by a traffic management center and utilized to establish a large-scale traffic monitoring and management system. With the improvement of stability and efficiency of this type of system, crash-proof and self-driving vehicles can be further developed, promoting the development of autonomous vehicle transportation systems.

Through establishment of international standards as well as cooperation across institutions, more revolutionary MR-related products and technologies may be developed and sustainable MR industries can be established, which will in turn enrich and upgrade the content of smart living in the coming 15 years and beyond.

\section{CONCLUSION AND FUTURE WORK}

The roadmap of MR sensors (non-recording) was developed in this paper. The past and current statuses of MR sensors were identified by analyzing the patent and publication statistics, and the timescales of MR sensors were established and predicted. MR devices are expected to proliferate with high sensing and operational performance in the area of biomedical applications, flexible electronics, PS and HCI, NDEM, and navigation and transportation. However, more investment on MR sensors is needed to reduce their costs in order to compete with Hall-effect sensors. Tens of millions of Hall effect devices are made each year, making the price of Hall-effect sensors lower than the MR 
sensors due to economy of scale [439]. The cost of MR sensor will continue to decrease as the sales volume increases. At high market volume of MR sensors, the cost difference between Hall sensor and MR sensors would be very small. In addition, MR sensors can provide unique performance that Hall elements cannot, which makes MR sensor more suitable for widespread use in the future.

\section{ACKNOWLEDGMENT}

This research is supported by the Seed Funding Program for Basic Research, Seed Funding Program for Applied Research and Small Project Funding Program from the University of Hong Kong, ITF Tier 3 funding (ITS/203/14, ITS/104/13, ITS/214/14), RGC-GRF grant (HKU 17210014, HKU 17204617), and University Grants Committee of Hong Kong (Contract No. AoE/P-04/08).

\section{REFERENCES}

[1] M. N. Baibich, J. M. Broto, A. Fert, F. N. Van Dau, F. Petroff, P. Etienne, G. Creuzet, A. Friederich, and J. Chazelas, "Giant Magnetoresistance of (001)Fe/(001)Cr Magnetic Superlattices," Physi. Rev. Lett., vol. 61, pp. 2472-2475, 1988.

[2] G. Binasch, P. Grünberg, F. Saurenbach, and W. Zinn, "Enhanced magnetoresistance in layered magnetic structures with antiferromagnetic interlayer exchange," Physi. Rev. B, vol. 39, p. 4828, 1989.

[3] M. Julliere, "Tunneling between ferromagnetic films," Phys. Lett. A, vol. 54, pp. 225-226, 1975/09/08 1975.

[4] B. Dieny, V. S. Speriosu, S. S. P. Parkin, B. A. Gurney, D. R. Wilhoit, and D. Mauri, "Giant magnetoresistive in soft ferromagnetic multilayers," Physi. Rev. B, vol. 43, pp. 1297-1300, 01/01/ 1991.

[5] J. M. Daughton, "GMR and SDT sensor applications," IEEE Trans. Magn., vol. 36, pp. 2773-2778, 2000.

[6] C. Giebeler, D. J. Adelerhof, A. E. T. Kuiper, J. B. A. van Zon, D. Oelgeschläger, and G. Schulz, "Robust GMR sensors for angle detection and rotation speed sensing," Sens. Actuators. A: Phys., vol. 91, pp. 16-20, 2001.

[7] P. Freitas, R. Ferreira, S. Cardoso, and F. Cardoso, "Magnetoresistive sensors," J. Phys.: Condens. Matter, vol. 19, p. $165221,2007$.

[8] I. Bakonyi and L. Péter, "Electrodeposited multilayer films with giant magnetoresistance (GMR): Progress and problems," Prog. Mater. Sci., vol. 55, pp. 107-245, 2010.

[9] J. Llandro, J. Palfreyman, A. Ionescu, and C. Barnes, "Magnetic biosensor technologies for medical applications: a review," Med. Biol. Eng. Comput., vol. 48, pp. 977-998, 2010.

[10] G. Xiao, "Magnetoresistive sensors based on magnetic tunneling junctions," Taylor \& Francis Group, Boca Raton, England, 2012.

[11] L. Jogschies, D. Klaas, R. Kruppe, J. Rittinger, P. Taptimthong, A Wienecke, L. Rissing, and M. C. Wurz, "Recent developments of magnetoresistive sensors for industrial applications," Sensors, vol. 15, pp. 28665-28689, 2015.

[12] M. D. Cubells-Beltrán, C. Reig, J. Madrenas, A. De Marcellis, J. Santos, S. Cardoso, and P. Freitas, "Integration of GMR Sensors with Different Technologies," Sensors, vol. 16, p. 939, 2016.

[13] A. Bocheux, C. Cavoit, M. Mouchel, C. Ducruet, R. Fons, P. Sabon, I.-L. Prejbeanu, and C. Baraduc, "High sensitivity magnetic field sensor for spatial applications," in 2016 IEEE SAS, Catania, Italy, 2016.

[14] M. Hämäläinen, R. Hari, R. J. Ilmoniemi, J. Knuutila, and O. V. Lounasmaa, "Magnetoencephalography - theory, instrumentation, and applications to noninvasive studies of the working human brain," Rev. Mod. Phys., vol. 65, p. 413, 1993.

[15] S. M. Powell, G. M. Petersen, A. J. Krush, S. Booker, J. Jen, F. M. Giardiello, S. R. Hamilton, B. Vogelstein, and K. W. Kinzler,
"Molecular diagnosis of familial adenomatous polyposis," New Engl. J. Med., vol. 329, pp. 1982-1987, 1993.

[16] D. R. Baselt, G. U. Lee, M. Natesan, S. W. Metzger, P. E. Sheehan, and R. J. Colton, "A biosensor based on magnetoresistance technology," Biosens. Bioelectron., vol. 13, pp. 731-739, 1998.

[17] H. Koch, "SQUID magnetocardiography: status and perspectives," IEEE Trans. Appl. Supercond., vol. 11, pp. 49-59, 2001.

[18] M. Miller, P. Sheehan, R. Edelstein, C. Tamanaha, L. Zhong, S. Bounnak, L. Whitman, and R. Colton, "A DNA array sensor utilizing magnetic microbeads and magnetoelectronic detection," $J$. Magn. Magn. Mater., vol. 225, pp. 138-144, 2001.

[19] D. Graham, H. Ferreira, J. Bernardo, P. Freitas, and J. Cabral, "Single magnetic microsphere placement and detection on-chip using current line designs with integrated spin valve sensors: Biotechnological applications," J. Appl. Phys., vol. 91, pp. 77867788, 2002.

[20] L. Lagae, R. Wirix-Speetjens, J. Das, D. Graham, H. Ferreira, P. Freitas, G. Borghs, and J. De Boeck, "On-chip manipulation and magnetization assessment of magnetic bead ensembles by integrated spin-valve sensors," J. Appl. Phys., vol. 91, pp. 7445-7447, 2002.

[21] M. Miller, G. Prinz, S.-F. Cheng, and S. Bounnak, "Detection of a micron-sized magnetic sphere using a ring-shaped anisotropic magnetoresistance-based sensor: a model for a magnetoresistancebased biosensor," Appl. Phys. Lett., vol. 81, pp. 2211-2213, 2002.

[22] I. Tavarozzi, S. Comani, C. Del Gratta, S. Di Luzio, G. L. Romani, S. Gallina, M. Zimarino, D. Brisinda, R. Fenici, and R. De Caterina, "Magnetocardiography: current status and perspectives. Part II: Clinical applications," Ital. Heart J., vol. 3, pp. 151-165, 2002.

[23] D. L. Graham, H. A. Ferreira, P. P. Freitas, and J. M. S. Cabral, "High sensitivity detection of molecular recognition using magnetically labelled biomolecules and magnetoresistive sensors," Biosens. Bioelectron., vol. 18, pp. 483-488, 2003.

[24] J. Rife, M. Miller, P. Sheehan, C. Tamanaha, M. Tondra, and L. Whitman, "Design and performance of GMR sensors for the detection of magnetic microbeads in biosensors," Sens. Actuators. A: Phys., vol. 107, pp. 209-218, 2003.

[25] L. M. Staudt, "Molecular diagnosis of the hematologic cancers," New Engl. J. Med., vol. 348, pp. 1777-1785, 2003.

[26] L. Ejsing, M. F. Hansen, A. K. Menon, H. Ferreira, D. Graham, and P. Freitas, "Planar Hall effect sensor for magnetic micro-and nanobead detection," Appl. Phys. Lett., vol. 84, pp. 4729-4731, 2004.

[27] D. L. Graham, H. A. Ferreira, and P. P. Freitas, "Magnetoresistivebased biosensors and biochips," Trends Biotechnol., vol. 22, pp. 455-462, 2004.

[28] M. Pannetier, C. Fermon, G. Le Goff, J. Simola, and E. Kerr, "Femtotesla magnetic field measurement with magnetoresistive sensors," Science, vol. 304, pp. 1648-1650, 2004.

[29] H. Ferreira, N. Feliciano, D. Graham, L. Clarke, M. Amaral, and P. Freitas, "Rapid DNA hybridization based on ac field focusing of magnetically labeled target DNA," Appl. Phys. Lett., vol. 87, p. 013901, 2005.

[30] H. A. Ferreira, D. L. Graham, N. Feliciano, L. A. Clarke, M. D. Amaral, and P. P. Freitas, "Detection of cystic fibrosis related DNA targets using AC field focusing of magnetic labels and spin-valve sensors," IEEE Trans. Magn., vol. 41, pp. 4140-4142, 2005.

[31] S. G. Grancharov, H. Zeng, S. Sun, S. X. Wang, S. O'Brien, C. Murray, J. Kirtley, and G. Held, "Bio-functionalization of monodisperse magnetic nanoparticles and their use as biomolecular labels in a magnetic tunnel junction based sensor," J. Phys. Chem. $B$, vol. 109, pp. 13030-13035, 2005.

[32] M. Pannetier, C. Fermon, G. Legoff, J. Simola, E. Kerr, M. Welling, and R. J. Wijngaarden, "Ultra-sensitive field sensors-an alternative to SQUIDs," IEEE Trans. Appl. Supercond., vol. 15, pp. 892-895, 2005.

[33] F. Cardoso, H. Ferreira, J. Conde, V. Chu, P. Freitas, D. Vidal, J. Germano, L. Sousa, M. Piedade, and B. Costa, "Diode/magnetic tunnel junction cell for fully scalable matrix-based biochip," J. Appl. Phys., vol. 99, p. 08B307, 2006.

[34] L. Lui, Y. Tan, K. Li, C. Sow, and S. O'Shea, "Detection of ferromagnetic particles using spin valve sensors," J. Appl. Phys., vol. 100, p. 044909, 2006.

[35] F. C. Paixao, R. de Moraes, M. Stelzer, L. A. Cora, M. F. Americo, U. Andreis, R. B. Oliveira, O. Baffa, and R. d. A. Jose, "A novel 
biomagnetic instrumentation with four magnetoresistive sensors to evaluate gastric motility," in the $29^{\text {th }}$ EMBS, Lyon, France, 2007. F. C. Paixao, F. M. Silva, R. d. A. Jose, and O. Baffa, "Magnetoresistive sensors in a new biomagnetic instrumentation for applications in gastroenterology," in he $29^{\text {th }}$ EMBS, Lyon, France, 2007.

[37] F. Cardoso, J. Germano, R. Ferreira, S. Cardoso, V. Martins, P. Freitas, M. Piedade, and L. Sousa, "Detection of $130 \mathrm{~nm}$ magnetic particles by a portable electronic platform using spin valve and magnetic tunnel junction sensors," J. Appl. Phys., vol. 103, p. 07A310, 2008

[38] V. C. Martins, F. A. Cardoso, J. Loureiro, M. Mercier, J. Germano, S. Cardoso, R. Ferreira, L. P. Fonseca, L. Sousa, M. S. Piedade, and P. P. Freitas, "Integrated Spintronic Platforms for Biomolecular Recognition Detection," AIP Conf. Proc., vol. 1025, pp. 150-175, 2008

[39] J. Germano, V. C. Martins, F. A. Cardoso, T. M. Almeida, L. Sousa, P. P. Freitas, and M. S. Piedade, "A portable and autonomous magnetic detection platform for biosensing," Sensors, vol. 9, pp. 4119-4137, 2009

[40] Y. Li, Y. Jing, X. Yao, B. Srinivasan, Y. Xu, C. Xing, and J.-P. Wang, "Biomarkers identification and detection based on GMR sensor and sub $13 \mathrm{~nm}$ magnetic nanoparticles," in the IEEE EMBC, Minneapolis, USA, 2009

[41] H. Liu, S. Li, L. Liu, L. Tian, and N. He, "Multiplex single nucleotide polymorphisms genotyping using solid-phase single base extension on magnetic nanoparticles," Anal. Boichem., vol. 386, pp. 126-128, 2009.

[42] J. Loureiro, C. Fermon, M. Pannetier-Lecoeur, G. Arrias, R. Ferreira, S. Cardoso, and P. Freitas, "Magnetoresistive detection of magnetic beads flowing at high speed in microfluidic channels," IEEE Trans. Magn., vol. 45, pp. 4873-4876, 2009.

[43] J. Loureiro, R. Ferreira, S. Cardoso, P. Freitas, J. Germano, C. Fermon, G. Arrias, M. Pannetier-Lecoeur, F. Rivadulla, and J. Rivas, "Toward a magnetoresistive chip cytometer: Integrated detection of magnetic beads flowing at $\mathrm{cm} / \mathrm{s}$ velocities in microfluidic channels," Appl. Phys. Lett., vol. 95, p. 034104, 2009.

[44] V. Martins, F. Cardoso, J. Germano, S. Cardoso, L. Sousa, M. Piedade, P. Freitas, and L. Fonseca, "Femtomolar limit of detection with a magnetoresistive biochip," Biosens. Bioelectron., vol. 24, pp. 2690-2695, 2009

[45] X. Xue, W. Xu, F. Wang, and X. Liu, "Multiplex single-nucleotide polymorphism typing by nanoparticle-coupled DNA-templated reactions," J. Am. Chem. Soc., vol. 131, pp. 11668-11669, 2009.

[46] V. C. Martins, J. Germano, F. A. Cardoso, J. Loureiro, S. Cardoso, L. Sousa, M. Piedade, L. P. Fonseca, and P. Freitas, "Challenges and trends in the development of a magnetoresistive biochip portable platform," J. Magn. Magn. Mater., vol. 322, pp. 1655-1663, 2010.

[47] M. Pannetier-Lecoeur, C. Fermon, H. Dyvorne, J. F. Jacquinot, H. Polovy, and A. L. Walliang, "Magnetoresistive-superconducting mixed sensors for biomagnetic applications," J. Magn. Magn. Mater., vol. 322, pp. 1647-1650, 2010.

[48] J. Amaral, S. Cardoso, P. Freitas, and A. Sebastião, "Toward a system to measure action potential on mice brain slices with local magnetoresistive probes," J. Appl. Phys., vol. 109, p. 07B308, 2011.

[49] M. A. DePristo, E. Banks, R. Poplin, K. V. Garimella, J. R. Maguire, C. Hartl, A. A. Philippakis, G. Del Angel, M. A. Rivas, and M. Hanna, "A framework for variation discovery and genotyping using next-generation DNA sequencing data," Nat. Genet., vol. 43, pp. 491-498, 2011.

[50] M. Pannetier-Lecoeur, L. Parkkonen, N. Sergeeva-Chollet, H. Polovy, C. Fermon, and C. Fowley, "Magnetocardiography with sensors based on giant magnetoresistance," Appl. Phys. Lett., vol. 98, p. 153705, 2011.

[51] P. Freitas, F. Cardoso, V. Martins, S. Martins, J. Loureiro, J. Amaral, R. Chaves, S. Cardoso, L. Fonseca, and A. Sebastião, "Spintronic platforms for biomedical applications," Lab Chip, vol. 12, pp. 546-557, 2012.

[52] R. Juárez-Aguirre, S. Domínguez-Nicolás, E. Manjarrez, J. Tapia, E. Figueras, H. Vázquez-Leal, L. Aguilera-Cortés, and A. HerreraMay, "Digital Signal Processing by Virtual Instrumentation of a MEMS Magnetic Field Sensor for Biomedical Applications," Sensors, vol. 13, p. 15068, 2013.
S. Oh, M. Jadhav, J. Lim, V. Reddy, and C. Kim, "An organic substrate based magnetoresistive sensor for rapid bacteria detection," Biosens. Bioelectron., vol. 41, pp. 758-763, 3/15/ 2013.

[54] C. M. Earhart, C. E. Hughes, R. S. Gaster, C. C. Ooi, R. J. Wilson, L. Y. Zhou, E. W. Humke, L. Xu, D. J. Wong, and S. B. Willingham, "Isolation and mutational analysis of circulating tumor cells from lung cancer patients with magnetic sifters and biochips," Lab Chip, vol. 14 , pp. 78-88, 2014.

[55] A. C. Fernandes, C. M. Duarte, F. A. Cardoso, R. Bexiga, S. Cardoso, and P. P. Freitas, "Lab-on-chip cytometry based on magnetoresistive sensors for bacteria detection in milk," Sensors, vol. 14, pp. 15496-15524, 2014.

[56] L. Caruso, "Giant magnetoresistance based sensors for local magnetic detection of neuronal currents," $\mathrm{PhD}$ dissertation, Université Pierre et Marie Curie-Paris VI, Paris, France, 2015.

[57] L. Ichkitidze, N. Bazaev, D. Telyshev, R. Preobrazhensky, and M. Gavrushina, "Magnetic field sensors in medical diagnostics," Biomed. Eng., vol. 48, pp. 305-309, 2015.

[58] L. Caruso, T. Wunderle, C. M. Lewis, J. Valadeiro, V. Trauchessec, J. Trejo-Rosillo, J. P. Amaral, J. Ni, C. Fermon, S. Cardoso, P. P. Freitas, P. Fries, and M. Pannetier-Lecoeur, "In vivo magnetic recording of neuronal activity," Neuron, vol. 95, pp. 1283-1291, 2017.

[59] L. Zhang, "3D Navigation for Endoscope by Magnetic Field," Proc. SPIE, 2001.

[60] G. Li, V. Joshi, R. L. White, S. X. Wang, J. T. Kemp, C. Webb, R. W. Davis, and S. Sun, "Detection of single micron-sized magnetic bead and magnetic nanoparticles using spin valve sensors for biological applications," J. Appl. Phys., vol. 93, pp. 7557-7559, 2003.

[61] L. Xu, H. Yu, M. S. Akhras, S.-J. Han, S. Osterfeld, R. L. White, N. Pourmand, and S. X. Wang, "Giant magnetoresistive biochip for DNA detection and HPV genotyping," Biosens. Bioelectron., vol. 24, pp. 99-103, 2008.

[62] R. S. Gaster, D. A. Hall, C. H. Nielsen, S. J. Osterfeld, H. Yu, K. E. Mach, R. J. Wilson, B. Murmann, J. C. Liao, and S. S. Gambhir, "Matrix-insensitive protein assays push the limits of biosensors in medicine," Nat. Med., vol. 15, pp. 1327-1332, 2009.

[63] T. G. Barroso, R. C. Martins, E. Fernandes, S. Cardoso, J. Rivas, and P. P. Freitas, "Detection of BCG bacteria using a magnetoresistive biosensor: A step towards a fully electronic platform for tuberculosis point-of-care detection," Biosens. Bioelectron., vol. 100, pp. 259-265, 2018.

[64] S. Parkin, K. Roche, and T. Suzuki, "Giant magnetoresistance in antiferromagnetic $\mathrm{Co} / \mathrm{Cu}$ multilayers grown on kapton," Japanese J. Appl. Phys., vol. 31, p. L1246, 1992.

[65] L. Piraux, J. George, J. Despres, C. Leroy, E. Ferain, R. Legras, K Ounadjela, and A. Fert, "Giant magnetoresistance in magnetic multilayered nanowires," Appl. Phys. Lett., vol. 65, pp. 2484-2486, 1994.

[66] S. Parkin, "Flexible giant magnetoresistance sensors," Appl. Phys. Lett., vol. 69, pp. 3092-3094, 1996.

[67] L. Baril, B. Gurney, D. Wilhoit, and V. Speriosu, "Magnetostriction in spin valves," J. Appl. Phys., vol. 85, pp. 5139-5141, 1999.

[68] F. Yan, G. Xue, and F. Wan, "A flexible giant magnetoresistance sensor prepared completely by electrochemical synthesis," J. Mater. Chem., vol. 12, pp. 2606-2608, 2002.

[69] S. Dokupil, M. T. Bootsmann, S. Stein, M. Löhndorf, and E. Quandt, "Positive/negative magnetostrictive GMR trilayer systems as strain gauges," J. Magn. Magn. Mater., vol. 290-291, pp. 795-799, 2005.

[70] T. Uhrmann, L. Bär, T. Dimopoulos, N. Wiese, M. Rührig, and A. Lechner, "Magnetostrictive GMR sensor on flexible polyimide substrates," J. Magn. Magn. Mater., vol. 307, pp. 209-211, 2006.

[71] Z. Guo, S. Park, H. T. Hahn, S. Wei, M. Moldovan, A. B. Karki, and D. P. Young, "Giant magnetoresistance behavior of an iron/carbonized polyurethane nanocomposite," Appl. Phys. Lett., vol. 90 , p. 053111, 2007.

[72] T. Santos, J. Lee, P. Migdal, I. Lekshmi, B. Satpati, and J. Moodera, "Room-temperature tunnel magnetoresistance and spin-polarized tunneling through an organic semiconductor barrier," Physi. Rev. Lett., vol. 98, p. 016601, 2007.

[73] Y. f. Chen, Y. Mei, R. Kaltofen, J. I. Mönch, J. Schumann, J. Freudenberger, H. J. Klauß, and O. G. Schmidt, "Towards Flexible Magnetoelectronics: Buffer - Enhanced and Mechanically Tunable 
GMR of Co/Cu Multilayers on Plastic Substrates," Adv. Mater., vol. 20, pp. 3224-3228, 2008.

[74] T. Wen, D. Liu, C. K. Luscombe, and K. M. Krishnan, "Granular magnetoresistance in cobalt/poly (3-hexylthiophene, 2, 5-diyl) hybrid thin films prepared by a wet chemical method," Appl. Phys. Lett., vol. 95, p. 082509, 2009.

[75] C. Barraud, C. Deranlot, P. Seneor, R. Mattana, B. Dlubak, S. Fusil, K. Bouzehouane, D. Deneuve, F. Petroff, and A. Fert, "Magnetoresistance in magnetic tunnel junctions grown on flexible organic substrates," Appl. Phys. Lett., vol. 96, p. 2502, 2010.

[76] M. Gobbi, F. Golmar, R. Llopis, F. Casanova, and L. E. Hueso, "Room - Temperature Spin Transport in C60 - Based Spin Valves," Adv. Mater., vol. 23, pp. 1609-1613, 2011.

[77] B. Li, C. Y. Kao, J. W. Yoo, V. N. Prigodin, and A. J. Epstein, "Magnetoresistance in an All - Organic - Based Spin Valve," Adv. Mater., vol. 23, pp. 3382-3386, 2011.

[78] M. Melzer, D. Makarov, A. Calvimontes, D. Karnaushenko, S. Baunack, R. Kaltofen, Y. Mei, and O. G. Schmidt, "Stretchable magnetoelectronics," Nano Lett., vol. 11, pp. 2522-2526, 2011.

[79] D. Karnaushenko, D. Makarov, C. Yan, R. Streubel, and O. G. Schmidt, "Printable giant magnetoresistive devices," Adv. Mater., vol. 24, pp. 4518-4522, 2012.

[80] M. Melzer, G. Lin, D. Makarov, and O. G. Schmidt, "Stretchable spin valves on elastomer membranes by predetermined periodic fracture and random wrinkling," Adv. Mater., vol. 24, pp. 64686472, 2012.

[81] M. Donolato, C. Tollan, J. M. Porro, A. Berger, and P. Vavassori, "Flexible and Stretchable Polymers with Embedded Magnetic Nanostructures," Adv. Mater., vol. 25, pp. 623-629, 2013.

[82] N. Gorjizadeh and S. Y. Quek, "Interface effects on tunneling magnetoresistance in organic spintronics with flexible amine-Au links," Nanotechnol., vol. 24, p. 415201, 2013.

[83] D. Makarov, D. Karnaushenko, and O. G. Schmidt, "Printable Magnetoelectronics," ChemPhysChem, vol. 14, pp. 1771-1776, 2013.

[84] S. Oh, J. S. Yu, J. Lim, M. Jadhav, T. M. Lee, D. Kim, and C. Kim, "Highly Flexible Magnetoelectronic Device Integrated With Embedded Ag Nanoparticle Electrode," IEEE Sens. J., vol. 13, pp. 3957-3961, 2013.

[85] A. Bedoya-Pinto, M. Donolato, M. Gobbi, L. E. Hueso, and P. Vavassori, "Flexible spintronic devices on Kapton," Appl. Phys. Lett., vol. 104, p. 062412, 2014.

[86] J. Valadeiro, J. Amaral, D. Leitao, A. Silva, J. Gaspar, M. Silva, M. Costa, M. Martins, F. Franco, and H. Fonseca, "Bending effect on magnetoresistive silicon probes," IEEE Trans. Magn., vol. 51, pp. $1-4,2015$.

[87] J. Gaspar, H. Fonseca, E. Paz, M. Martins, J. Valadeiro, S. Cardoso, R. Ferreira, and P. P. Freitas, "Flexible magnetoresistive sensors designed for conformal integration," IEEE Trans. Magn., vol. 53, pp. 1-4, 2017.

[88] U. Ausserlechner, "The optimum layout for giant magneto-resistive angle sensors," IEEE Sens. J., vol. 10, pp. 1571-1582, 2010.

[89] M. R. Johnson, "The challenges in applying magnetroesistive sensors on the'Curiosity'Rover," California Institute of Technology, CA, USA, 2013.

[90] S. T. Wu, J. Y. Chen, and S. H. Wu, "A rotary encoder with an eccentrically mounted ring magnet," IEEE Trans. Instrum. Meas., vol. 63, pp. 1907-1915, 2014.

[91] R. Hahn, T. Schmidt, R. Slatter, B. Olberts, and F. Romera, "Magneto-resistive angular sensors for space applications: results of breadboard and EQM testing and lessons learned," in Proc. ESMATS, Hatfield, UK, 2017.

[92] J. H. Kim, S. J. Son, Y. C. Lim, T. G. Kim, E. S. Kim, and Y. J. Ryoo, "Fuzzy Steering Controller for Outdoor Autonomous Mobile Robot using MR sensor," J. Korean Inst. Intell. Syst., vol. 12, pp. 27-32, 2002.

[93] M. Kurita, T. Yamazaki, H. Kohira, M. Matsumoto, R. Tsuchiyama, J. Xu, T. Harada, Y. Inoue, L. Su, and K. Kato, "An active-head slider with a piezoelectric actuator for controlling flying height," IEEE Trans. Magn., vol. 38, pp. 2102-2104, 2002.

[94] Y. L. Chen, S. C. Chen, W. L. Chen, and J. F. Lin, "A head orientated wheelchair for people with disabilities," Disabil. Rehabil., vol. 25, pp. 249-253, 2003/01/01 2003.
S. Bonnet, P. Couturier, F. Favre-Reguillon, and R. Guillemaud, "Evaluation of postural stability by means of a single inertial sensor," in the $26^{\text {th }}$ IEEE EMBS, San Francisco, CA, USA, 2004

[96] J. Bae and R. M. Voyles, "Wearable joystick for gloves-on human/computer interaction," in Proc. SPIE, pp. 62300N-62300N10,2006

[97] I. C. T. Santos, J. M. R. S. Tavares, J. G. Mendes, and M. P. F. Paulo, "Acquisition and analysis of 3D mandibular movement using a device based on electromagnetic sensors and a neural network," $J$. Med. Eng. Technol., vol. 33, pp. 437-441, 2009.

[98] V. T. Jensen and W. H. Huber, "Surgical navigation system with wireless magnetoresistance tracking sensors," ed: Google Patents, 2010.

[99] V. T. Jensen and W. H. Huber, "Surgical navigation system with magnetoresistance sensors," ed: Google Patents, 2013.

[100] L. P. Simmons and J. S. Welsh, "Light Weight Finger Tracking Using a Single Point Permanent Magnetic Field Reference," in CIMSim, Seoul, South Korea, 2013.

[101] L. P. Simmons and J. S. Welsh, "Particle filter based finger tracking utilising magnetoresistive sensors," in IEEE/ASME AIM, Besancon, France, 2014.

[102] T. Chady, "Evaluation of stress loaded steel samples using GMR magnetic field sensor," IEEE Sens. J., vol. 2, pp. 488-493, 2002.

[103] R. Rempt, "Scanning with magnetoresistive sensors for subsurface corrosion," AIP Conf. Proc., vol. 615, pp. 1771-1778, 2002.

[104] B. Wincheski, J. Simpson, M. Namkung, D. Perey, E. Scales, and R. Louie, "Development of Giant Magnetoresistive inspection system for detection of deep fatigue cracks under airframe fasteners," AIP Conf. Proc., vol. 615, pp. 1007-1014, 2002.

[105] S. M. Amin and B. F. Wollenberg, "Toward a smart grid: power delivery for the 21st century," IEEE Power Energy Mag., vol. 3, pp. 34-41, 2005.

[106] H. Kirkham, "Current measurement methods for the smart grid," in IEEE PES, Calgary, Alberta Canada, 2009.

[107] P. McDaniel and S. McLaughlin, "Security and privacy challenges in the smart grid," IEEE Secur. Priv., vol. 7, 2009.

[108] H. Farhangi, "The path of the smart grid," IEEE Power Energy Mag., vol. 8, pp. 18-28, 2010.

[109] M. Bukhari, "A Large scale seismic monitoring paradigm and accelerometry technique based on the giant magnetoresistance effect," Malaysian J. Sci., vol. 30, pp. 182-195, 2011.

[110] X. Sun, K. Lui, K. Wong, W. Lee, Y. Hou, Q. Huang, and P. W. T. Pong, "Novel application of magnetoresistive sensors for highvoltage transmission-line monitoring," IEEE Trans. Magn., vol. 47, pp. 2608-2611, 2011.

[111] Q. Huang, W. Zhen, and P. W. T. Pong, "A novel approach for fault location of overhead transmission line with noncontact magneticfield measurement," IEEE Trans. Power Del., vol. 27, pp. 11861195, 2012.

[112] L. Y. Liu, S. B. Jiang, T. L. Yeh, H. C. Yeh, J. Y. Liu, Y. H. Hsu, and J.-Y. Peng, "The Magneto-Resistive Magnetometer of BCU on the Tatiana-2 Satellite," Terr. Atmos. Ocean Sci., vol. 23, 2012.

[113] Y. Ouyang, J. He, J. Hu, and S. X. Wang, "A current sensor based on the giant magnetoresistance effect: Design and potential smart grid applications," Sensors, vol. 12, pp. 15520-15541, 2012.

[114] P. Pai, L. Chen, F. K. Chowdhury, and M. Tabib-Azar, "Nonintrusive electric power sensors for smart grid," in IEEE Sensors, Taipei, Taiwan, 2012.

[115] T. J. Phiri, "Correlation between SQUID and fluxgate magnetometer data for geomagnetic storms," Stellenbosch: M.S. thesis, Dept. Eletron. Eng., Stellenbosch University, Stellenbosch, 7599, South Africa, 2013.

[116] X. Sun, Q. Huang, Y. Hou, L. Jiang, and P. W. T. Pong, "Noncontact operation-state monitoring technology based on magnetic-field sensing for overhead high-voltage transmission lines," IEEE Trans. Power Del., vol. 28, pp. 2145-2153, 2013.

[117] X. Sun, C. K. Poon, G. Chan, C. L. Sum, W. K. Lee, L. Jiang, and P. W. T. Pong, "Operation-state monitoring and energization-status identification for underground power cables by magnetic field sensing," IEEE Sens. J., vol. 13, pp. 4527-4533, 2013.

[118] Q. Huang, Y. Song, X. Sun, L. Jiang, and P. W. T. Pong, "Magnetics in Smart Grid," IEEE Trans. Magn., vol. 50, pp. 1-7, 2014.

[119] J. S. Donnal and S. B. Leeb, "Noncontact power meter," IEEE Sens. J., vol. 15, pp. 1161-1169, 2015. 
[120] K. Zhu, W. K. Lee, and P. W. T. Pong, "Non-Contact CapacitiveCoupling-Based and Magnetic-Field-Sensing-Assisted Technique for Monitoring Voltage of Overhead Power Transmission Lines," IEEE Sens. J., 2017.

[121] K. Zhu, W. Han, W. K. Lee, and P. W. T. Pong, "On-Site NonInvasive Current Monitoring of Multi-Core Underground Power Cables With a Magnetic-Field Sensing Platform at a Substation," IEEE Sens. J., vol. 17, pp. 1837-1848, 2017.

[122] W. Du, H. Nguyen, A. Dutt, and K. Scallion, "Design of a GMR sensor array system for robotic pipe inspection," in 2010 IEEE Sensors, 2010.

[123] M. J. Caruso, "Applications of magnetoresistive sensors in navigation systems," Honeywell Inc., Morris Plains, NJ, USA, Tech. Rep. 0148-7191, 1997.

[124] S. V. Marshall, "Vehicle detection using a magnetic field sensor," IEEE Trans. Veh. Technol., vol. 27, pp. 65-68, 1978.

[125] D. L. Striker and R. J. Hampo, "Method of position sensing utilizing giant magneto resistance elements and solid state switch array," ed: Google Patents, 1999.

[126] K. S. Park, C. S. Kim, Y. J. Lee, J. I. Bae, and M. H. Lee, "Development of an unmanned vehicle driving system by MR sensor," in IEEE IECON, Nagoya, Aichi, Japan, 2000.

[127] K.-M. Lenssen, D. Adelerhof, H. Gassen, A. Kuiper, G. Somers, and J. Van Zon, "Robust giant magnetoresistance sensors," Sens. Actuators. A: Phys., vol. 85, pp. 1-8, 2000.

[128] C. Giebeler, D. Adelerhof, A. Kuiper, J. Van Zon, D. Oelgeschläger, and G. Schulz, "Robust GMR sensors for angle detection and rotation speed sensing," Sens. Actuators. A: Phys., vol. 91, pp. 1620, 2001.

[129] J. Pelegri, J. Alberola, and V. Llario, "Vehicle detection and car speed monitoring system using GMR magnetic sensors," in in IEEE IECON, Sevilla, Spain, 2002.

[130] J. Včelák, P. Ripka, J. Kubik, A. Platil, and P. Kašpar, "AMR navigation systems and methods of their calibration," Sens. Actuators. A: Phys., vol. 123, pp. 122-128, 2005.

[131] M. A. Cordoba, "Attitude and heading refernce system i-ahrs for the efigenia autonomous unmanned aerial vehicles uav based on mems sensor and a neural network strategy for attitude estimation," in IEEE MED, Athens, Greece, 2007.

[132] H.-f. YANG and Q. ZHOU, "New Vehicle Detector Based on SCM and Magnetoresistive Transducer [J]," J. Chongqing Inst. Technol. (Natural Sci.), vol. 11, p. 005, 2008.

[133] Y.-C. Lai, S.-S. Jan, and F.-B. Hsiao, "Development of a low-cost attitude and heading reference system using a three-axis rotating platform," Sensors, vol. 10, pp. 2472-2491, 2010.

[134] M. Michelena, I. Arruego, J. Oter, and H. Guerrero, "COTS-based wireless magnetic sensor for small satellites," IEEE Trans. Aerosp. Electron. Syst., vol. 46, pp. 542-557, 2010.

[135] R. Sanz, A. B. Fernández, J. A. Dominguez, B. Martín, and M. D. Michelena, "Gamma irradiation of magnetoresistive sensors for planetary exploration," Sensors, vol. 12, pp. 4447-4465, 2012.

[136] H. S. Guo, B. Yan, and Z. D. Wu, "Attitude Measurement System with Low Power Consumption for Underwater Observation Platform," Torpedo Technol., vol. 3, p. 007, 2013.

[137] Z. Zhao, Y. Chen, and W. Yi, "Design of wireless vehicle detector based on AMR sensor [J]," Electron. Meas. Technol., vol. 1, p. 002, 2013.

[138] X. Zhou, "Vehicle location estimation based on a magnetic sensor array," in IEEE SAS, Galveston, TX, USA, 2013.

[139] B. Yang and Y. Lei, "Vehicle detection and classification for lowspeed congested traffic with anisotropic magnetoresistive sensor," IEEE Sens. J., vol. 15, pp. 1132-1138, 2015.

[140] M. J. Caruso, "Applications of magnetoresistive sensors in navigation systems," Prog. Technol., vol. 72, pp. 159-168, 1998.

[141] S. I. R. Arias, D. R. Muñoz, S. Cardoso, R. Ferreira, and P. J. P. de Freitas, "Total ionizing dose (TID) evaluation of magnetic tunnel junction (MTJ) current sensors," Sens. Actuators. A: Phys., vol. 225, pp. 119-127, 2015.

[142] L. Gao, A. L. Porter, J. Wang, S. Fang, X. Zhang, T. Ma, W. Wang, and L. Huang, "Technology life cycle analysis method based on patent documents," Technol. Forecast. Soc. Chang., vol. 80, pp. 398-407, 2013.

[143] J. C. Mankins, "Technology Readiness Levels, NASA Advanced Concepts Office, Office of Space Access and Technology," NASA, Washington, D.C., USA, Tech. Rep., 1995.
[144] Y. Kajikawa, J. Yoshikawa, Y. Takeda, and K. Matsushima, "Tracking emerging technologies in energy research: Toward a roadmap for sustainable energy," Technol. Forecast. Soc. Chang., vol. 75, pp. 771-782, 2008.

[145] M. Bengisu and R. Nekhili, "Forecasting emerging technologies with the aid of Science and technology databases," Technol. Forecast. Soc. Chang., vol. 73, pp. 835-844, 2006.

[146] A. J. Clark, L. W. Lake, and T. W. Patzek, "Production forecasting with logistic growth models," in SPE ATCE, Denver, CO, USA, 2011.

[147] J. P. Martino, "A review of selected recent advances in technological forecasting," Technol. Forecast. Soc. Chang., vol. 70, pp. 719-733, 2003.

[148] M. J. Caruso, T. Bratland, C. H. Smith, and R. Schneider, "A new perspective on magnetic field sensing," Sensors (Peterborough, NH), vol. 15, pp. 34-47, 1998.

[149] J. Lenz and A. S. Edelstein, "Magnetic sensors and their applications," Sensors J., IEEE, vol. 6, pp. 631-649, 2006.

[150] C. Reig, M.-D. Cubells-Beltrán, and D. Ramírez Muñoz, "Magnetic field sensors based on giant magnetoresistance (GMR) technology: Applications in electrical current sensing," Sensors, vol. 9, pp. 79197942, 2009.

[151] Q. Herreros, H. Dyvorne, P. Campiglio, G. Jasmin-Lebras, A. Demonti, M. Pannetier-Lecoeur, and C. Fermon, "Very low field Magn. Reson. Imaging with spintronic sensors," Rev. Sci. Instrum., vol. 84, p. 095116, 2013.

[152] A. Hirohata, H. Sukegawa, H. Yanagihara, I. Zutic, T. Seki, S. Mizukami, and R. Swaminathan, "Roadmap for Emerging Magnetic Materials for Spintronic Device Applications," IEEE Trans. Magn., vol. 51(10), pp. 1-11, 2015.

[153] H. Pfleiderer, "Magnetodiode model," Solid-State Electronics, vol. 15, pp. 335-353, 1972.

[154] C. S. Roumenin, "Bipolar magnetotransistor sensors. An invited review," Sens. Actuators. A: Phys., vol. 24, pp. 83-105, 1990.

[155] R. E. Popovic, Z. Randjelovic, and D. Manic, "Integrated Hall-effect magnetic sensors," Sens. Actuators. A: Phys., vol. 91, pp. 46-50, 2001.

[156] D. Drung, "High-Tc and low-Tc dc SQUID electronics," Supercond. Sci. Technol., vol. 16, p. 1320, 2003.

[157] I. Žutić, J. Fabian, and S. Das Sarma, "Spintronics: Fundamentals and applications," Rev. Mod. Phys., vol. 76, pp. 323-410, 04/23/ 2004.

[158] D. Budker and M. Romalis, "Optical magnetometry," Nat. Phys., vol. 3, pp. 227-234, 2007.

[159] A. Bratkovsky, "Spintronic effects in metallic, semiconductor, metal-oxide and metal-semiconductor heterostructures," Rep. Prog. Phys., vol. 71, p. 026502, 2008.

[160] X. Huo, J. Wang, and M. Ghovanloo, "A magneto-inductive sensor based wireless tongue-computer interface," IEEE Trans. Neural Syst. Rehabil. Eng., vol. 16, pp. 497-504, 2008.

[161] P. Zu, C. Chiu Chan, T. Gong, Y. Jin, W. Chang Wong, and X. Dong, "Magneto-optical fiber sensor based on bandgap effect of photonic crystal fiber infiltrated with magnetic fluid," Appl. Phys. Lett., vol. 101, p. 241118, 2012.

[162] X. Li, K. W. Lin, H. Y. Liu, D. H. Wei, G. Li, and P. W. T. Pong, "Effect of field cooling process and ion-beam bombardment on the exchange bias of $\mathrm{NiCo} /(\mathrm{Ni}, \mathrm{Co})$ O bilayers," Thin Solid Films, vol. 570, pp. 383-389, 2014.

[163] X. Li, C. Zheng, Y. Zhou, H. Kubota, S. Yuasa, and P. W. T. Pong, "Spin-torque diode with tunable sensitivity and bandwidth by outof-plane magnetic field," Appl. Phys. Lett., vol. 108, p. 232407, 2016.

[164] X. Li, Y. Zhou, C. Zheng, P. Chan, M. Chan, and P. W. T. Pong, "Field-angle and DC-bias dependence of spin-torque diode in giant magnetoresistive microstripe," Appl. Phys. Lett., vol. 109, p. 192402, 2016.

[165] C. Zheng, T. C. Lan, C. Shueh, R. D. Desautels, J. van Lierop, K.W. Lin, and P. W. T. Pong, "Effect of ion-beam bombardment on microstructural and magnetic properties of Ni80Fe20/ $\alpha-\mathrm{Fe} 2 \mathrm{O} 3$ thin films," Japanese J. Appl. Phys., vol. 53, p. 06JB03, 2014.

[166] C. Zheng, K. W. Lin, C. H. Liu, H. F. Hsu, C. W. Leung, W. H. Chen, T. H. Wu, R. D. Desautels, J. van Lierop, and P. W. T. Pong, "Microstructural and magnetic characterization of ion-beam bombarded [Ni $80 \mathrm{Fe} 20-\mathrm{Cr}] 50$ thin films," Vac., vol. 118, pp. 8589, 2015. 

Lierop, and P. W. T. Pong, "Influence of different stoichiometric Croxides created via oxygen ion-beam bombardment on the magnetism of the NiFe/Cr-oxide exchange-biased systems," Phys. Lett. A, 2017

[168] P. Ripka and M. Janosek, "Advances in magnetic field sensors," IEEE Sens. J., vol. 10, pp. 1108-1116, 2010.

[169] S. X. Wang and G. Li, "Advances in Giant Magnetoresistance Biosensors With Magnetic Nanoparticle Tags: Review and Outlook," IEEE Trans. Magn., vol. 44, pp. 1687-1702, 2008.

[170] F. N. Van Dau, A. Schuhl, J. Childress, and M. Sussiau, "Magnetic sensors for nanotesla detection using planar Hall effect," Sens. Actuators. A: Phys., vol. 53, pp. 256-260, 1996.

[171] A. Bilotti, G. Monreal, and R. Vig, "Monolithic magnetic Hall sensor using dynamic quadrature offset cancellation," IEEE J. SolidState Circuits, vol. 32, pp. 829-836, 1997.

[172] J.-B. Kammerer, L. Hébrard, V. Frick, P. Poure, and F. Braun, "Horizontal Hall effect sensor with high maximum absolute sensitivity," IEEE Sens. J., vol. 3, pp. 700-707, 2003.

[173] F. Primdahl, "The fluxgate mechanism, part I: The gating curves of parallel and orthogonal fluxgates," IEEE Trans. Magn., vol. 6, pp. 376-383, 1970

[174] F. Primdahl, "The fluxgate magnetometer," J. Phys. E: Sci. Instrum., vol. 12, p. 241, 1979

[175] P. Ripka, "Review of fluxgate sensors," Sens. Actuators. A: Phys. vol. 33, pp. 129-141, 1992.

[176] S. Choi, S. Kawahito, Y. Matsumoto, M. Ishida, and Y. Tadokoro, "An integrated micro fluxgate magnetic sensor," Sens. Actuators. A: Phys., vol. 55, pp. 121-126, 1996.

[177] P. Ripka, "Advances in fluxgate sensors," Sens. Actuators. A: Phys. vol. 106, pp. 8-14, 2003.

[178] J. Zimmerman, "Sensitivity Enhancement of Superconducting Quantum Interference Devices through the Use of Fractional - Turn Loops," J. Appl. Phys., vol. 42, pp. 4483-4487, 1971.

[179] J. P. Cleuziou, W. Wernsdorfer, V. Bouchiat, T. Ondarçuhu, and M. Monthioux, "Carbon nanotube superconducting quantum interference device," Nat. Nanotechnol., vol. 1, pp. 53-59, 2006.

[180] R. Fagaly, "Superconducting quantum interference device instruments and applications," Rev. Sci. Instrum., vol. 77, p. 101101, 2006.

[181] D. Drung, C. Abmann, J. Beyer, A. Kirste, M. Peters, F. Ruede, and T. Schurig, "Highly sensitive and easy-to-use SQUID sensors," IEEE Trans. Appl. Supercond., vol. 17, pp. 699-704, 2007.

[182] P. Zu, C. C. Chan, W. S. Lew, Y. Jin, Y. Zhang, H. F. Liew, L. H. Chen, W. C. Wong, and X. Dong, "Magneto-optical fiber sensor based on magnetic fluid," Opt. Lett., vol. 37, pp. 398-400, 2012.

[183] M. Ghanaatshoar and M. Zamani, "Magneto-optical magnetic field sensors based on compact magnetophotonic crystals," J. Supercond. Novel Magn., vol. 28, pp. 1365-1370, 2015.

[184] A. Garzarella, M. Shinn, and D. H. Wu, "Responsivity optimization in magneto-optic sensors based on ferromagnetic materials," Appl. Opt., vol. 54, pp. 7904-7911, 2015.

[185] R. Amat, H. García-Miquel, D. Barrera, G. V. Kurlyandskaya, and S. Sales, "Magneto-optical sensor based on fiber Bragg gratings and a magnetostrictive material," Key Eng. Mater., vol. 644, pp. 232$235,2015$.

[186] R. García, E. Blanco, and M. Domínguez, "Development of a magneto-optical sensor prototype to measure current by means of the induced magnetic field," Sens. Actuators. A: Phys., vol. 249, pp. 231-241, 2016.

[187] H. Kasper, B. Hess, and N. Dieringer, "A precise and inexpensive magnetic field search coil system for measuring eye and head movements in small laboratory animals," J. NeuroSci. Methods, vol. 19, pp. 115-124, 1987.

[188] M. Oka and M. Enokizono, "A detection of backside crack using rotational magnetic flux sensor with search coils," IEEE Trans. Magn., vol. 32, pp. 4968-4970, 1996.

[189] O. Bergamin, D. S. Zee, D. C. Roberts, K. Landau, A. G. Lasker, and D. Straumann, "Three-dimensional Hess screen test with binocular dual search coils in a three-field magnetic system," Invest. Ophthalmol. Vis. Sci., vol. 42, pp. 660-667, 2001.

[190] H. Seran and P. Fergeau, "An optimized low-frequency three-axis search coil magnetometer for space research," Rev. Sci. Instrum., vol. 76, p. 044502, 2005
[191] A. Roux, O. Le Contel, C. Coillot, A. Bouabdellah, B. De La Porte, D. Alison, S. Ruocco, and M. C. Vassal, "The search coil magnetometer for THEMIS," in the THEMIS Mission, Springer, New York, NY, USA, 2009.

[192] K. Kawashima, T. Kohzawa, H. Yoshida, and K. Mohri, "Magnetoinductive effect in tension-annealed amorphous wires and MI sensors," IEEE Trans. Magn., vol. 29, pp. 3168-3170, 1993.

[193] M. Jagiella, S. Fericean, R. Droxler, and A. Dorneich, "New magneto-inductive sensing principle and its implementation in sensors for industrial applications," in Sensors, 2004. Proceedings of IEEE, 2004, pp. 1020-1023.

[194] A. Boukhenoufa, C. P. Dolabdjian, and D. Robbes, "Highsensitivity giant magneto-inductive magnetometer characterization implemented with a low-frequency magnetic noise-reduction technique," IEEE Sens. J., vol. 5, pp. 916-923, 2005.

[195] X. Huo and M. Ghovanloo, "A wireless pharmaceutical compliance monitoring system based on magneto-inductive sensors," IEEE Sens. J., vol. 7, pp. 1711-1719, 2007.

[196] K. Mohri, T. Uchiyama, L. Shen, C. Cai, and L. Panina, "Amorphous wire and CMOS IC-based sensitive micro-magnetic sensors (MI sensor and SI sensor) for intelligent measurements and controls," J. Magn. Magn. Mater., vol. 249, pp. 351-356, 2002.

[197] B. Li, A. M. Morsy, and J. Kosel, "Optimization of autonomous magnetic field sensor consisting of giant magnetoimpedance sensor and surface acoustic wave transducer," IEEE Trans. Magn., vol. 48, pp. 4324-4327, 2012

[198] M. Ipatov, V. Zhukova, A. Zhukov, and J. Gonzalez, "Expanding the longitudinal magnetoimpedance sensor range by direct bias current," J. Appl. Phys., vol. 113, p. 203902, 2013.

[199] B. Li, M. N. Kavaldzhiev, and J. Kosel, "Flexible magnetoimpedance sensor," J. Magn. Magn. Mater., vol. 378, pp. 499-505, 2015.

[200] O. Lutes, P. Nussbaum, and O. Aadland, "Sensitivity limits in SOS magnetodiodes," IEEE Trans. Electron Devices., vol. 27, pp. 2156$2157,1980$.

[201] C. S. Roumenin, "2D magnetodiode sensors based on SOS technology," Sens. Actuators. A: Phys., vol. 54, pp. 584-588, 1996. A. Abramov and I. Gorbatyi, "Special features of the magnetodiode effect in multivalley semiconductors at low temperatures," Semiconductors, vol. 36, pp. 793-799, 2002.

[203] A. Abramov and I. Gorbatyi, "Intervalley redistribution of electrons at low temperatures and the magnetodiode effect," Semiconductors, vol. 37, pp. 1053-1056, 2003.

[204] R. S. Popovic and R. Widmer, "Magnetotransistor in CMOS technology," IEEE Trans. Electron Devices., vol. 33, pp. 13341340, 1986.

[205] A. Nathan, K. Maenaka, W. Allegretto, H. Baltes, and T. Nakamura, "The Hall effect in integrated magnetotransistors," IEEE Trans. Electron Devices., vol. 36, pp. 108-117, 1989.

[206] H. Trujillo, A. Nagy, and J. Cruz, "Influence of topology on the response of lateral magnetotransistors," Sens. Actuators. A: Phys., vol. 45, pp. 179-182, 1994.

[207] C. Roumenin, P. Nikolova, and A. Ivanov, "Magnetotransistor sensors with amperometric output," Sens. Actuators. A: Phys., vol. 69, pp. 16-20, 1998.

[208] E. B. Alexandrov and V. A. Bonch-Bruevich, "Optically pumped atomic magnetometers after three decades," Optical Engineering, vol. 31, 1992.

[209] G. Bison, R. Wynands, and A. Weis, "A laser-pumped magnetometer for the mapping of human cardiomagnetic fields," Appl. Phys. B, vol. 76, pp. 325-328, 2003.

[210] E. Alexandrov, "Recent progress in optically pumped magnetometers," Ohys. Scr., vol. 2003, p. 27, 2003.

[211] I. Kominis, T. Kornack, J. Allred, and M. Romalls, "A subfemtotesla multichannel atomic magnetometer," Nature, vol. 422, p. 596, 2003.

[212] H. Tang, R. Kawakami, D. Awschalom, and M. Roukes, "Giant planar Hall effect in epitaxial (Ga, Mn) As devices," Physi. Rev. Lett., vol. 90, p. 107201, 2003.

[213] D. Kim, C. Kim, B. Park, and C. Park, "Thickness dependence of planar Hall resistance and field sensitivity in $\mathrm{NiO}(30 \mathrm{~nm}) / \mathrm{NiFe}(\mathrm{t})$ bilayers," J. Magn. Magn. Mater., vol. 215, pp. 585-588, 2000.

[214] D. Kim, B. Park, and C. Kim, "Optimization of planar Hall resistance using biaxial currents in a $\mathrm{NiO} / \mathrm{NiFe}$ bilayer: 
Enhancement of magnetic field sensitivity," J. Appl. Phys., vol. 88, pp. 3490-3494, 2000.

[215] N. Thanh, M. Chun, N. Ha, K. Kim, C. Kim, and C. Kim, "Thickness dependence of exchange anisotropy in NiFe/IrMn bilayers studied by Planar Hall Effect," J. Magn. Magn. Mater., vol. 305, pp. 432435, 2006.

[216] N. Thanh, M. Chun, J. Schmalhorst, G. Reiss, K. Kim, and C. Kim, "Magnetizing angle dependence of planar Hall resistance in spinvalve structure," J. Magn. Magn. Mater., vol. 304, pp. e84-e87, 2006.

[217] N. Thanh, L. Tu, N. Ha, C. Kim, C. Kim, K. Shin, and B. Parvatheeswara Rao, "Thickness dependence of parallel and perpendicular anisotropic resistivity in $\mathrm{Ta} / \mathrm{NiFe} / \mathrm{IrMn} / \mathrm{Ta}$ multilayer studied by anisotropic magnetoresistance and planar Hall effect," $J$. Appl. Phys., vol. 101, p. 053702, 2007.

[218] T. Q. Hung, J.-R. Jeong, D.-Y. Kim, N. H. Duc, and C. Kim, "Hybrid planar Hall-magnetoresistance sensor based on tilted crossjunction," J. Phys. D: Appl. Phys., vol. 42, p. 055007, 2009.

[219] V. A. Dediu, L. E. Hueso, I. Bergenti, and C. Taliani, "Spin routes in organic semiconductors," Nat. Mater., vol. 8, pp. 707-716, 2009.

[220] C. Zheng, R. D. Shull, P. Chen, and P. W. T. Pong, "Kondo effect in magnetic tunnel junctions with an $\mathrm{AlO} x$ tunnel barrier," Phys. Lett. A, vol. 380, pp. 2237-2241, 2016.

[221] C. Zheng, X. Li, R. Shull, P. Chen, and P. W. T. Pong, "Comprehensive noise characterisation of magnetic tunnel junction sensors for optimising sensor performance and temperature detection," Mater. Res. Innovations, vol. 19, pp. 53-57, 2015.

[222] M. Pannetier-Lecoeur, C. Fermon, H. Dyvorne, J. F. Jacquinot, H. Polovy, and A. L. Walliang, "Magnetoresistive-superconducting mixed sensors for biomagnetic applications," J. Magn. Magn. Mater., vol. 322, pp. 1647-1650, 2010.

[223] K. Kobayashi and H. Akimoto, "TMR film and head technologies," Fujitsu Sci. Tech. J., vol. 42, pp. 139-148, 2006.

[224] C. J. Cochrane, J. Blacksberg, M. A. Anders, and P. M. Lenahan, "Vectorized magnetometer for space applications using electrical readout of atomic scale defects in silicon carbide," Sci. Rep., vol. 6, p. $37077,2016$.

[225] S. Sordo-Ibáñez, B. Piñero-García, M. Muñoz-Díaz, A. RagelMorales, J. Ceballos-Cáceres, L. Carranza-Gonzáez, S. EspejoMeana, A. Arias-Drake, J. Ramos-Martos, and J. M. MoraGutiérrez, "A front-end ASIC for a 3-d magnetometer for space applications by using anisotropic magnetoresistors," IEEE Trans. Magn., vol. 51, pp. 1-4, 2015.

[226] S. Leitner, A. Valavanoglou, P. Brown, C. Hagen, W. Magnes, B. J. Whiteside, C. M. Carr, M. Delva, and W. Baumjohann, "Design of the magnetoresistive magnetometer for ESA's SOSMAG project," IEEE Trans. Magn., vol. 51, pp. 1-4, 2015.

[227] M. Díaz-Michelena, R. Sanz, M. Cerdán, and A. Fernández, "Calibration of QM-MOURA three-axis magnetometer and gradiometer," Geosci. Instrum. Methods Data Syst., vol. 4, p. 1, 2015.

[228] M. Archer, T. Horbury, P. Brown, J. Eastwood, T. Oddy, B. Whiteside, and J. Sample, "The MAGIC of CINEMA: first in-flight Science results from a miniaturised anisotropic magnetoresistive magnetometer," Ann. Geophys, 2015, pp. 725-735.

[229] P. Brown, B. Whiteside, T. Beek, P. Fox, T. Horbury, T. Oddy, M. Archer, J. Eastwood, D. Sanz-Hernández, and J. Sample, "Space magnetometer based on an anisotropic magnetoresistive hybrid sensor," Rev. Sci. Instrum., vol. 85, p. 125117, 2014.

[230] P. Brown, T. Beek, C. Carr, H. O'Brien, E. Cupido, T. Oddy, and T. Horbury, "Magnetoresistive magnetometer for space Science applications," Meas. Sci. Technol., vol. 23, p. 025902, 2012.

[231] A. Persson, H. Nguyen, F. Riddar, and G. Thornell, "Micro- and nanostructured magnetic field sensor for space applications," in IEEE TRANSDUCERS, Denver, CO, USA, 2009.

[232] M. Díaz-Michelena, "Small magnetic sensors for space applications," Sensors, vol. 9, pp. 2271-2288, 2009.

[233] J. Wells, L. Stras, and T. Jeans, "Canada's smallest satellite: The canadian advanced nanospace experiment (CanX-1)," University of Toronto, Toronto, Ontario, Canada, Tech. Rep., 2002.

[234] K. Zhu, W. K. Lee, and P. W. T. Pong, " Fault line identification of HVDC transmission line by frequency spectrum correlation based on capacitive coupling and magnetic-field sensing," IEEE Trans Magn, vol. 99, pp. 1-5, 2018.
[235]

F. C. Paixao, F. M. Silva, R. d. A. Jose, and O. Baffa, "Magnetoresistive sensors in a new biomagnetic instrumentation for applications in gastroenterology," in the IEEE MBS, Lyon, France, 2007.

[236] R. R. Minor and D. W. Rowe, "Utilization of a magnetic sensor to compensate a MEMS-IMU/GPS and de-spin strapdown on rolling missiles," ed: Google Patents, 2001.

[237] Z. Aiyun, Y. Kui, Y. Zhigang, and Z. Haibing, "Research and application of a robot orientation sensor," in IEEE RISSP, Changsha, Hunan, China, 2003.

[238] C. S. Kim, J. Y. Choi, S. P. Hong, M. H. Lee, J. I. Bae, and F. Harashima, "H/sup/spl infin//steering control for the unmanned vehicle system," in IEEE IECON, Denver, CO, USA, 2001.

[239] P. J. Prado, "Single sided imaging sensor," Magn. Reson. Imaging, vol. 21, pp. 397-400, 2003

[240] T. Hirose, "Electronic Apparatus and Method of Detecting Housing Direction," ed: Google Patents, 2005.

[241] A. Balakrishnan, "Trace interconnect array having increased bandwidth by selective etching of traces and dielectric substrate," ed: Google Patents, 2002.

[242] M. Tondra, J. M. Daughton, D. Wang, R. S. Beech, A. Fink, and J. A. Taylor, "Picotesla field sensor design using spin-dependent tunneling devices," J. Appl. Phys., vol. 83, pp. 6688-6690, 1998.

[243] S.-H. Liou, X. Yin, S. E. Russek, R. Heindl, F. Da Silva, J. Moreland, D. P. Pappas, L. Yuan, and J. Shen, "Picotesla magnetic sensors for low-frequency applications," IEEE Trans. Magn., vol. 47, pp. 3740-3743, 2011.

[244] Honeywell, "Nanopower Series", 2018. Available: https://sensing.honeywell.com/sensors/position-sensorics/nanopower-series. [Accessed: Oct-15-2018].

[245] X. Yin, R. Skomski, D. Sellmyer, S. H. Liou, S. E. Russek, E. R. Evarts, J. Moreland, A. Edelstein, L. Yuan, and M. Yan, "Adjusting magnetic nanostructures for high-performance magnetic sensors," $J$. Appl. Phys., vol. 115, p. 17E528, 2014.

[246] K.-M. H. Lenssen, M. E. Lambrechts, and G. J. Vos, "Graphical display input device with magnetic field sensors," ed: Google Patents, 2006.

[247] B. Ahn, K. Park, H. Lee, E. I. S. Lorenzo, K. H. Rha, and J. Kim, "Robotic palpation system for prostate cancer detection," in the IEEE BioRob, Tokyo, Japan, 2010.

[248] Y.-F. Liu, X. Yin, Y. Yang, D. Ewing, P. J. D. Rego, and S.-H. Liou, "Tunneling magnetoresistance sensors with different coupled free layers," AIP Adv., vol. 7, p. 056666, 2017.

[249] J. P. Valadeiro, J. Amaral, D. C. Leitao, R. Ferreira, S. Freitas Cardoso, and P. J. Freitas, "Strategies for pTesla Field Detection Using Magnetoresistive Sensors With a Soft Pinned Sensing Layer," IEEE Trans. Magn., vol. 51, pp. 1-4, 2015.

[250] S. Cardoso, L. Gameiro, D. C. Leitao, F. Cardoso, R. Ferreira, E. $\mathrm{Paz}$, and P. P. Freitas, "Magnetic tunnel junction sensors with pTesla sensitivity for biomedical imaging," Proc. SPIE, 2013, pp. 87631A87631A-8.

[251] R. C. Chaves, P. P. Freitas, B. Ocker, and W. Maass, "MgO based picotesla field sensors," J. Appl. Phys., vol. 103, pp. 07E93107E931-3, 2008.

[252] E. Paz, S. Serrano-Guisan, R. Ferreira, and P. P. Freitas, "Room temperature direct detection of low frequency magnetic fields in the $100 \mathrm{pT} / \mathrm{Hz} 0.5$ range using large arrays of magnetic tunnel junctions," J. Appl. Phys., vol. 115, 2014.

[253] D. W. Guo, F. A. Cardoso, R. Ferreira, E. Paz, S. Cardoso, and P. P. Freitas, "MgO-based magnetic tunnel junction sensors array for non-destructive testing applications," J. Appl. Phys., vol. 115, p. 17E513, 2014.

[254] S. Cardoso, D. Leitao, L. Gameiro, F. Cardoso, R. Ferreira, E. Paz, and P. Freitas, "Magnetic tunnel junction sensors with pTesla sensitivity," Microsyst. Technol., vol. 20, pp. 793-802, 2014. G. Yu, J. Feng, H. Kurt, H. Liu, X. Han, and J. Coey, "Field sensing in $\mathrm{MgO}$ double barrier magnetic tunnel junctions with a superparamagnetic Co50Fe50 free layer," J. Appl. Phys., vol. 111, p. 113906, 2012.

[256] K. Fujiwara, M. Oogane, S. Yokota, T. Nishikawa, H. Naganuma, and Y. Ando, "Fabrication of magnetic tunnel junctions with a bottom synthetic antiferro-coupled free layers for high sensitive magnetic field sensor devices," J. Appl. Phys., vol. 111, pp. -, 2012. 

low aspect ratio linear magnetic tunnel junctions with a soft-pinned sensing layer," IEEE Trans. Magn., vol. 48, pp. 3719-3722, 2012.

[258] H. Duan, H. W. Tseng, Y. Li, and R. B. van Dover, "Improvement of the low-frequency sensitivity of $\mathrm{MgO}$-based magnetic tunnel junctions by annealing," J. Appl. Phys., vol. 109, p. 113917, 2011.

[259] H. Polovy, R. Guerrero, J. Scola, M. Pannetier-Lecoeur, C. Fermon, G. Feng, K. Fahy, S. Cardoso, J. Almeida, and P. Freitas, "Noise of MgO-based magnetic tunnel junctions," J. Magn. Magn. Mater., vol. 322, pp. 1624-1627, 2010.

[260] R. Guerrero, M. Pannetier-Lecoeur, C. Fermon, S. Cardoso, R. Ferreira, and P. P. Freitas, "Low frequency noise in arrays of magnetic tunnel junctions connected in series and parallel," J. Appl. Phys., vol. 105, pp. 113922-5, 2009.

[261] C. Albon, A. Weddemann, A. Auge, K. Rott, and A. Hütten, "Tunneling magnetoresistance sensors for high resolutive particle detection," Appl. Phys. Lett., vol. 95, 2009.

[262] Y. Lee, J. Hayakawa, S. Ikeda, F. Matsukura, and H. Ohno, "Effect of electrode composition on the tunnel magnetoresistance of pseudo-spin-valve magnetic tunnel junction with a $\mathrm{MgO}$ tunnel barrier," Appl. Phys. Lett., vol. 90, pp. 212507-212507-3, 2007.

[263] R. Chaves, P. Freitas, B. Ocker, and W. Maass, "Low frequency picotesla field detection using hybrid $\mathrm{MgO}$ based tunnel sensors," Appl. Phys. Lett., vol. 91, pp. 102504-102504-3, 2007.

[264] S. Yuasa, A. Fukushima, H. Kubota, Y. Suzuki, and K. Ando, "Giant tunneling magnetoresistance up to $410 \%$ at room temperature in fully epitaxial $\mathrm{Co} / \mathrm{MgO} / \mathrm{Co}$ magnetic tunnel junctions with bcc Co (001) electrodes," Appl. Phys. Lett., vol. 89, pp. 042505-042505-3, 2006.

[265] S. Yuasa, T. Katayama, T. Nagahama, A. Fukushima, H. Kubota, Y. Suzuki, and K. Ando, "Giant tunneling magnetoresistance in fully epitaxial body-centered-cubic $\mathrm{Co} / \mathrm{MgO} / \mathrm{Fe}$ magnetic tunnel junctions," Appl. Phys. Lett., vol. 87, p. 222508, 2005.

[266] K. Tsunekawa, D. D. Djayaprawira, M. Nagai, H. Maehara, S. Yamagata, N. Watanabe, S. Yuasa, Y. Suzuki, and K. Ando, "Giant tunneling magnetoresistance effect in low-resistance $\mathrm{CoFeB} /$ $\mathrm{MgO}(001) / \mathrm{CoFeB}$ magnetic tunnel junctions for read-head applications," Appl. Phys. Lett., vol. 87, p. 072503, 2005.

[267] N. Tezuka, N. Ikeda, F. Mitsuhashi, and S. Sugimoto, "Improved tunnel magnetoresistance of magnetic tunnel junctions with Heusler Co2FeA10.5Si0.5 electrodes fabricated by molecular beam epitaxy," Appl. Phys. Lett., vol. 94, p. 162504, 2009.

[268] N. Tezuka, N. Ikeda, A. Miyazaki, S. Sugimoto, M. Kikuchi, and K. Inomata, "Tunnel magnetoresistance for junctions with epitaxial full-Heusler Co2FeA10.5Si0.5 electrodes with B2 and L21 structures," Appl. Phys. Lett., vol. 89, p. 112514, 2006.

[269] D. Wang, C. Nordman, J. M. Daughton, Z. Qian, and J. Fink, "70\% TMR at room temperature for SDT sandwich junctions with $\mathrm{CoFeB}$ as free and reference layers," IEEE Trans. Magn., vol. 40, pp. 22692271,2004

[270] S. Ikeda, J. Hayakawa, Y. Ashizawa, Y. Lee, K. Miura, H. Hasegawa, M. Tsunoda, F. Matsukura, and H. Ohno, "Tunnel magnetoresistance of $604 \%$ at $300 \mathrm{~K}$ by suppression of Ta diffusion in $\mathrm{CoFeB} / \mathrm{MgO} / \mathrm{CoFeB}$ pseudo-spin-valves annealed at high temperature," Appl. Phys. Lett., vol. 93, p. 2508, 2008.

[271] H. Sukegawa, Y. Miura, S. Muramoto, S. Mitani, T. Niizeki, T. Ohkubo, K. Abe, M. Shirai, K. Inomata, and K. Hono, "Enhanced tunnel magnetoresistance in a spinel oxide barrier with cation-site disorder," Physi. Rev. B, vol. 86, p. 184401, 2012.

[272] R. C. Sousa, J. J. Sun, V. Soares, P. P. Freitas, A. Kling, M. F. da Silva, and J. C. Soares, "Large tunneling magnetoresistance enhancement by thermal anneal," Appl. Phys. Lett., vol. 73, pp. 3288-3290, 1998

[273] X. Liu and G. Xiao, "Thermal annealing effects on low-frequency noise and transfer behavior in magnetic tunnel junction sensors," $J$. Appl. Phys., vol. 94, pp. 6218-6220, 2003.

[274] R. Stearrett, W. Wang, L. Shah, A. Gokce, J. Xiao, and E. Nowak, "Evolution of barrier-resistance noise in $\mathrm{CoFeB} / \mathrm{MgO} / \mathrm{CoFeB}$ tunnel junctions during annealing," J. Appl. Phys., vol. 107, p. 064502, 2010.

[275] W. K. Park, J. S. Moodera, J. Taylor, M. Tondra, J. M. Daughton, A. Thomas, and H. Bruckl, "Noise properties of magnetic and nonmagnetic tunnel junctions," J. Appl. Phys., vol. 93, pp. 7020$7022,2003$.
R. Guerrero, F. Aliev, R. Villar, J. Hauch, M. Fraune, G. Güntherodt, K. Rott, H. Brückl, and G. Reiss, "Low-frequency noise and tunneling magnetoresistance in $\mathrm{Fe}(110) / \mathrm{MgO}(111) / \mathrm{Fe}$ (110) epitaxial magnetic tunnel junctions," Appl. Phys. Lett., vol. 87, p. 042501, 2005.

[277] J. Almeida, P. Wisniowski, and P. Freitas, "Low-Frequency Noise in $\mathrm{MgO}$ Magnetic Tunnel Junctions: Hooge's Parameter Dependence on Bias Voltage," IEEE Trans. Magn., vol. 44, pp. 2569-2572, 2008.

[278] T. Miyazaki and N. Tezuka, "Giant magnetic tunneling effect in Fe/Al2O3/Fe junction," J. Magn. Magn. Mater., vol. 139, pp. L231L234, 1995.

[279] J. S. Moodera, L. R. Kinder, T. M. Wong, and R. Meservey, "Large magnetoresistance at room temperature in ferromagnetic thin film tunnel junctions," Physi. Rev. Lett., vol. 74, p. 3273, 1995.

[280] J. S. Moodera and L. R. Kinder, "Ferromagnetic-insulatorferromagnetic tunneling: Spin - dependent tunneling and large magnetoresistance in trilayer junctions," J. Appl. Phys., vol. 79, pp. 4724-4729, 1996

[281] W. Gallagher, S. S. Parkin, Y. Lu, X. Bian, A. Marley, K. Roche, R. Altman, S. Rishton, C. Jahnes, and T. Shaw, "Microstructured magnetic tunnel junctions," J. Appl. Phys., vol. 81, pp. 3741-3746, 1997.

[282] M. Tsunoda, K. Nishikawa, S. Ogata, and M. Takahashi, "60\% magnetoresistance at room temperature in $\mathrm{Co}-\mathrm{Fe} / \mathrm{Al}-\mathrm{O} / \mathrm{Co}-\mathrm{Fe}$ tunnel junctions oxidized with $\mathrm{Kr}-\mathrm{O} 2$ plasma," Appl. Phys. Lett., vol. 80, pp. 3135-3137, 2002.

[283] X. Liu, C. Ren, and G. Xiao, "Magnetic tunnel junction field sensors with hard-axis bias field," J. Appl. Phys., vol. 92, pp. 4722-4725, 2002.

[284] X.-F. Han, M. Oogane, H. Kubota, Y. Ando, and T. Miyazaki, "Fabrication of high-magnetoresistance tunnel junctions using Co75Fe25 ferromagnetic electrodes," Appl. Phys. Lett., vol. 77, pp. 283-285, 2000.

[285] S. Yuasa, T. Nagahama, A. Fukushima, Y. Suzuki, and K. Ando, "Giant room-temperature magnetoresistance in single-crystal $\mathrm{Fe} / \mathrm{MgO} / \mathrm{Fe}$ magnetic tunnel junctions," Nat. Mater., vol. 3, pp. 868871, 12//print 2004.

[286] S. S. Parkin, C. Kaiser, A. Panchula, P. M. Rice, B. Hughes, M. Samant, and S.-H. Yang, "Giant tunnelling magnetoresistance at room temperature with $\mathrm{MgO}$ (100) tunnel barriers," Nat. Mater., vol. 3, pp. 862-867, 2004.

[287] M. Bowen, V. Cros, F. Petroff, A. Fert, C. Martínez Boubeta, J. L. Costa-Krämer, J. V. Anguita, A. Cebollada, F. Briones, J. M. de Teresa, L. Morellón, M. R. Ibarra, F. Güell, F. Peiró, and A. Cornet, "Large magnetoresistance in $\mathrm{Fe} / \mathrm{MgO} / \mathrm{FeCo}(001)$ epitaxial tunnel junctions on GaAs(001)," Appl. Phys. Lett., vol. 79, pp. 1655-1657, 2001.

[288] S. Ishida, T. Masaki, S. Fujii, and S. Asano, "Theoretical search for half-metalliic films of Co2MnZ (Z . Si, Ge)," Phys. B: Condens. Matter., vol. 245, pp. 1-8, 1998.

[289] Y. Sakuraba, J. Nakata, M. Oogane, H. Kubota, Y. Ando, A. Sakuma, and T. Miyazaki, "Huge spin-polarization of L21-ordered Co2MnSi epitaxial Heusler alloy film," Japanese J. Appl. Phys., vol. 44, p. L1100, 2005.

[290] W. Wang, M. Przybylski, W. Kuch, L. Chelaru, J. Wang, Y. Lu, J. Barthel, H. Meyerheim, and J. Kirschner, "Magnetic properties and spin polarization of Co $2 \mathrm{MnSi}$ Heusler alloy thin films epitaxially grown on GaAs (001)," Physi. Rev. B, vol. 71, p. 144416, 2005.

[291] G. M. Müller, J. Walowski, M. Djordjevic, G.-X. Miao, A. Gupta, A. V. Ramos, K. Gehrke, V. Moshnyaga, K. Samwer, and J. Schmalhorst, "Spin polarization in half-metals probed by femtosecond spin excitation," Nat. Mater., vol. 8, p. 56, 2009.

[292] T. Ishikawa, T. Marukame, H. Kijima, K.-I. Matsuda, T. Uemura, M. Arita, and M. Yamamoto, "Spin-dependent tunneling characteristics of fully epitaxial magnetic tunneling junctions with a full-Heusler alloy Co2MnSi thin film and a $\mathrm{MgO}$ tunnel barrier," Appl. Phys. Lett., vol. 89, p. 192505, 2006.

[293] K. Inomata, S. Okamura, R. Goto, and N. Tezuka, "Large tunneling magnetoresistance at room temperature using a Heusler alloy with the B2 structure," Japanese J. Appl. Phys., vol. 42, p. L419, 2003.

[294] S. Kämmerer, A. Thomas, A. Hütten, and G. Reiss, "Co2MnSi Heusler alloy as magnetic electrodes in magnetic tunnel junctions," Appl. Phys. Lett., vol. 85, pp. 79-81, 2004. 
D. Ebke, J. Schmalhorst, N.-N. Liu, A. Thomas, G. Reiss, and A. Hütten, "Large tunnel magnetoresistance in tunnel junctions with Co2MnSi / Co2FeSi multilayer electrode," Appl. Phys. Lett., vol. 89, p. 162506, 2006.

[296] R. Shan, H. Sukegawa, W. H. Wang, M. Kodzuka, T. Furubayashi, T. Ohkubo, S. Mitani, K. Inomata, and K. Hono, "Demonstration of Half-Metallicity in Fermi-Level-Tuned Heusler Alloy $\mathrm{Co}_{2} \mathrm{Fe} \mathrm{Al}_{0.5} \mathrm{Si}_{0.5}$ at Room Temperature," Physi. Rev. Lett., vol. 102, p. 246601, 2009.

[297] W. Wang, H. Sukegawa, R. Shan, S. Mitani, and K. Inomata, "Giant tunneling magnetoresistance up to $330 \%$ at room temperature in sputter deposited $\mathrm{Co} 2 \mathrm{Fe} \mathrm{Al} / \mathrm{MgO} / \mathrm{CoFe}$ magnetic tunnel junctions," Appl. Phys. Lett., vol. 95, p. 182502, 2009.

[298] S. Hakamata, T. Ishikawa, T. Marukame, K.-i. Matsuda, T. Uemura, M. Arita, and M. Yamamoto, "Improved tunnel magnetoresistance characteristics of magnetic tunnel junctions with a Heusler alloy thin film of $\mathrm{Co} 2 \mathrm{Mn} \mathrm{Ge}$ and a $\mathrm{MgO}$ tunnel barrier," J. Appl. Phys., vol. 101, p. 09J513, 2007.

[299] T. Marukame, T. Ishikawa, S. Hakamata, K.-i. Matsuda, T. Uemura, and M. Yamamoto, "Highly spin-polarized tunneling in fully epitaxial $\mathrm{Co} 2 \mathrm{Cr} 0.6 \mathrm{Fe} 0.4 \mathrm{Al} / \mathrm{MgO} / \mathrm{Co} 50 \mathrm{Fe} 50$ magnetic tunnel junctions with exchange biasing," Appl. Phys. Lett., vol. 90, p. 012508, 2007.

[300] T. Ishikawa, S. Hakamata, K.-i. Matsuda, T. Uemura, and M. Yamamoto, "Fabrication of fully epitaxial Co2 $\mathrm{MnSi} / \mathrm{MgO}$ / Co2MnSi magnetic tunnel junctions," J. Appl. Phys., vol. 103, p. 07A919, 2008.

[301] S. Tsunegi, Y. Sakuraba, M. Oogane, K. Takanashi, and Y. Ando, "Large tunnel magnetoresistance in magnetic tunnel junctions using a Co2MnSi Heusler alloy electrode and a MgO barrier," Appl. Phys. Lett., vol. 93, p. 112506, 2008.

[302] W. Wang, H. Sukegawa, and K. Inomata, "Temperature dependence of tunneling magnetoresistance in epitaxial magnetic tunnel junctions using a Co $2 \mathrm{FeAl}$ Heusler alloy electrode," Physi. Rev. B, vol. 82, p. 092402, 2010

[303] D. D. Djayaprawira, K. Tsunekawa, M. Nagai, H. Maehara, S. Yamagata, N. Watanabe, S. Yuasa, Y. Suzuki, and K. Ando, "230\% room-temperature magnetoresistance in $\mathrm{CoFeB} / \mathrm{MgO} / \mathrm{CoFeB}$ magnetic tunnel junctions," Appl. Phys. Lett., vol. 86, 2005.

[304] K. H. J. Buschow and P. G. van Engen, "Magnetic and magnetooptical properties of heusler alloys based on aluminium and gallium," J. Magn. Magn. Mater., vol. 25, pp. 90-96, 1981.

[305] H. Sukegawa, S. Mitani, T. Ohkubo, K. Inomata, and K. Hono, "Low-resistive monocrystalline Mg-Al-O barrier magnetic tunnel junctions for spin-transfer magnetization switching," Appl. Phys. Lett., vol. 103, p. 142409, 2013.

[306] T. Scheike, H. Sukegawa, T. Furubayashi, Z. Wen, K. Inomata, T. Ohkubo, K. Hono, and S. Mitani, "Lattice-matched magnetic tunnel junctions using a Heusler alloy $\mathrm{Co} 2 \mathrm{FeAl}$ and a cation-disorder spinel Mg-Al-O barrier," Appl. Phys. Lett., vol. 105, p. 242407, 2014.

[307] M. Tsunoda, R. Chiba, and K. Kabara, "Fabrication of MgAl2O4 tunnel barrier by radio frequency-sputtering method and magnetoresistance effect through it with $\mathrm{Fe}$ or $\mathrm{Fe} 4 \mathrm{~N}$ ferromagnetic electrode," J. Appl. Phys., vol. 117, p. 17D703, 2015.

[308] N. Takahashi, T. Kawai, T. Yanase, T. Shimada, and T. Nagahama, "Investigation of epitaxial growth and tunnel magnetoresistance effects in magnetic tunnel junctions including spinel ferrite layers," Japanese J. Appl. Phys., vol. 54, p. 118003, 2015.

[309] H. Sukegawa, H. Xiu, T. Ohkubo, T. Furubayashi, T. Niizeki, W. Wang, S. Kasai, S. Mitani, K. Inomata, and K. Hono, "Tunnel magnetoresistance with improved bias voltage dependence in lattice-matched $\mathrm{Fe} /$ spinel MgAl2O4/Fe(001) junctions," Appl. Phys. Lett., vol. 96, p. 212505, 2010.

[310] J. Valadeiro, D. Leitao, S. Cardoso, and P. Freitas, "Improved efficiency of tapered magnetic flux concentrators with double layer architecture," IEEE Trans. Magn., 2017.

[311] X. Sun, L. Jiang, and P. W. T. Pong, "Magnetic flux concentration at micrometer scale," Microelectron. Eng., vol. 111, pp. 77-81, 2013.

[312] P. Leroy, C. Coillot, A. Roux, and G. Chanteur, "Optimization of the Shape of Magnetic Field Concentrators to Improve the Sensitivity of Hall Sensors," Technisches Messen, vol. 73, pp. 339349, 2006.
[313] W. Egelhoff Jr, P. W. T. Pong, J. Unguris, R. McMichael, E. Nowak, A. Edelstein, J. Burnette, and G. Fischer, "Critical challenges for picoTesla magnetic-tunnel-junction sensors," Sens. Actuators. A: Phys., vol. 155, pp. 217-225, 2009.

[314] C. Fermon, "Applications II: The Secret Revolution of Magnetic Sensors," in Nanosci. Nanotechnol., ed: Springer, 2016, pp. 103109.

[315] A. V. Silva, D. C. Leitao, J. Valadeiro, J. Amaral, P. P. Freitas, and S. Cardoso, "Linearization strategies for high sensitivity magnetoresistive sensors," Eur. Phys. J. Appl. Phys., vol. 72, p. $10601,2015$.

[316] Y. Lee, C. Chao, L. Li, Y. Suen, L. Horng, T.-H. Wu, C. Chang, and $\mathrm{J}$. Wu, "Magnetic tunnel junction based out-of-plane field sensor with perpendicular magnetic anisotropy in reference layer," J. Appl. Phys., vol. 117, p. 17A320, 2015.

[317] D. C. Leitao, L. Gameiro, A. V. Silva, S. Cardoso, and P. P. Freitas, "Field detection in spin valve sensors using $\mathrm{CoFeB} / \mathrm{Ru}$ syntheticantiferromagnetic multilayers as magnetic flux concentrators," IEEE Trans. Magn., vol. 48, pp. 3847-3850, 2012.

[318] R. J. Janeiro, L. Gameiro, A. Lopes, S. Cardoso, R. Ferreira, E. Paz, and P. P. Freitas, "Linearization and field detectivity in magnetic tunnel junction sensors connected in series incorporating $16 \mathrm{~nm}-$ thick NiFe free layers," IEEE Trans. Magn., vol. 48, pp. 4111-4114, 2012.

[319] A. Guedes, G. Jaramillo, C. Buffa, G. Vigevani, S. Cardoso, D. Leitao, P. Freitas, and D. Horsley, "Towards picoTesla magnetic field detection using a GMR-MEMS hybrid device," IEEE Trans. Magn., vol. 48, pp. 4115-4118, 2012.

[320] J. Chen, N. Carroll, J. Feng, and J. Coey, "Yoke-shaped MgObarrier magnetic tunnel junction sensors," Appl. Phys. Lett., vol. 101, p. 262402, 2012.

[321] R. Chaves, S. Cardoso, R. Ferreira, and P. Freitas, "Low aspect ratio micron size tunnel magnetoresistance sensors with permanent magnet biasing integrated in the top lead," J. Appl. Phys., vol. 109, p. 07E506, 2011.

[322] J. Almeida and P. Freitas, "Field detection in MgO magnetic tunnel junctions with superparamagnetic free layer and magnetic flux concentrators," J. Appl. Phys., vol. 105, pp. 07E722-07E722, 2009.

[323] S.-H. Liou, R. Zhang, S. E. Russek, L. Yuan, S. T. Halloran, and D. P. Pappas, "Dependence of noise in magnetic tunnel junction sensors on annealing field and temperature," J. Appl. Phys., vol. 103, p. 07E920, 2008.

[324] A. Guedes, S. Patil, S. Cardoso, V. Chu, J. Conde, and P. Freitas, "Hybrid magnetoresistive / microelectromechanical devices for static field modulation and sensor 1 / f noise cancellation," J. Appl. Phys., vol. 103, p. 07E924, 2008.

[325] D. Mazumdar, X. Liu, B. Schrag, W. Shen, M. Carter, and G. Xiao, "Thermal stability, sensitivity, and noise characteristics of $\mathrm{MgO}-$ based magnetic tunnel junctions," J. Appl. Phys., vol. 101, p. 09B502, 2007.

[326] A. Guedes, J. Almeida, S. Cardoso, R. Ferreira, and P. Freitas, "Improving magnetic field detection limits of spin valve sensors using magnetic flux guide concentrators," IEEE Trans. Magn., vol. 43, pp. 2376-2378, 2007.

[327] J. G. Deak, Z. Zhou, and W. Shen, "Tunneling magnetoresistance sensor with pT level 1/f magnetic noise," AIP Adv., vol. 7, p. 056676, 2017.

[328] A. Edelstein, G. Fischer, M. Pedersen, E. Nowak, S. F. Cheng, and C. Nordman, "Progress toward a thousandfold reduction in $1 / \mathrm{f}$ noise in magnetic sensors using an ac microelectromechanical system flux concentrator," J. Appl. Phys., vol. 99, p. 08B317, 2006. [329] W. Tian, J. Hu, M. Pan, D. Chen, and J. Zhao, "Flux concentration and modulation based magnetoresistive sensor with integrated planar compensation coils," Rev. Science Instrum., vol. 84, p. 035004, 2013

[330] J. Hu, W. Tian, J. Zhao, M. Pan, D. Chen, and G. Tian, "Remedying magnetic hysteresis and 1/f noise for magnetoresistive sensors," Appl. Phys. Lett., vol. 102, p. 054104, 2013.

[331] X. Li, J. Hu, W. Chen, L. Yin, and X. Liu, "A Novel High-Precision Digital Tunneling Magnetic Resistance-Type Sensor for the Nanosatellites' Space Application," Micromachines, vol. 9, p. 121, 2018. 
[332] S. Wang, T. He, and Y. Zhang, "Research on a Superconducting Magnetic Flux Concentrator for a GMI-Based Mixed Sensor," IEEE Trans. Appl. Supercond., vol. 24, pp. 1-5, 2014.

[333] SBIR STTR, "Low Cost High Sensitivity Superconducting Magnetometers and Gradiometers", 2017. Available: https://www.sbir.gov/sbirsearch/detail/1208053.[Accessed: Oct-152018].

[334] B. T. Matthias, T. H. Geballe, L. D. Longinotti, E. Corenzwit, G. W. Hull, R. H. Willens, and J. P. Maita, "Superconductivity at 20 Degrees Kelvin," Science, vol. 156, pp. 645-646, 1967.

[335] G. W. Webb, L. J. Vieland, R. E. Miller, and A. Wicklund, "Superconductivity above $20^{\circ} \mathrm{K}$ in stoichiometric Nb3Ga," Solid State Commun., vol. 9, pp. 1769-1773, 10/15/ 1971.

[336] J. Gavaler, "Superconductivity in Nb-Ge films above $22 \mathrm{~K}, "$ Appl. Phys. Lett., vol. 23, pp. 480-482, 1973.

[337] J. Muller, "A15-type superconductors," Rep. Prog. Phys., vol. 43, p. 641, 1980.

[338] M. R. Beasley and T. H. Geballe, "Superconducting materials," Phys. Today, vol. 37, pp. 60-68, 1984.

[339] G. Stewart, Z. Fisk, J. Willis, and J. Smith, "Possibility of coexistence of bulk superconductivity and spin fluctuations in UPt3," in Ten Years of Superconductivity: 1980-1990, ed: Springer, 1984, pp. 85-88.

[340] J. G. Bednorz and K. A. Müller, "Possible high T c superconductivity in the $\mathrm{Ba}-\mathrm{La}-\mathrm{Cu}-\mathrm{O}$ system," in Ten Years of Superconductivity: 1980-1990, ed: Springer, 1986, pp. 267-271.

[341] N. A. Stutzke, S. E. Russek, D. P. Pappas, and M. Tondra, "Lowfrequency noise measurements on commercial magnetoresistive magnetic field sensors," J. Appl. Phys., vol. 97, p. 10Q107, 2005.

[342] C. Chu, P. Hor, R. Meng, L. Gao, and Z. Huang, "Superconductivity at $52.5 \mathrm{~K}$ in the lanthanum-barium-copper-oxide system," Science, vol. 235, pp. 567-570, 1987.

[343] C. Chu, P. Hor, R. Meng, L. Gao, Z. Huang, Wang, and YQ, "Evidence for superconductivity above $40 \mathrm{~K}$ in the La-Ba-Cu-O compound system," Physi. Rev. Lett., vol. 58, p. 405, 1987.

[344] M.-K. Wu, J. R. Ashburn, C. J. Torng, P. H. Hor, R. L. Meng, L. Gao, Z. J. Huang, Y. Wang, and a. Chu, "Superconductivity at $93 \mathrm{~K}$ in a new mixed-phase $\mathrm{Y}-\mathrm{Ba}-\mathrm{Cu}-\mathrm{O}$ compound system at ambient pressure," Physi. Rev. Lett., vol. 58, p. 908, 1987.

[345] K. Tanigaki, T. W. Ebbesen, S. Saito, J. Mizuki, J. S. Tsai, Y. Kubo, and S. Kuroshima, "Superconductivity at $33 \mathrm{~K}$ in CsxRbyC60," Nature, vol. 352, pp. 222-223, 1991.

[346] S. N. Putilin, E. V. Antipov, O. Chmaissem, and M. Marezio, "Superconductivity at $94 \mathrm{~K}$ in $\mathrm{HgBa}_{2} \mathrm{Cu}_{4+\delta}$," Nature, vol. 362, pp. 226-228, 1993.

[347] G. Chen, Z. Li, D. Wu, G. Li, W. Hu, J. Dong, P. Zheng, J. Luo, and N. Wang, "Superconductivity at $41 \mathrm{~K}$ and its competition with spindensity-wave instability in layered $\mathrm{CeO} 1-\mathrm{x}$ F x FeAs," Physi. Rev. Lett., vol. 100, p. $247002,2008$.

[348] X. H. Chen, T. Wu, G. Wu, R. H. Liu, H. Chen, and D. F. Fang, "Superconductivity at 43[thinsp]K in SmFeAsO1-xFx," Nature, vol. 453, pp. 761-762, 2008.

[349] Y. Kamihara, T. Watanabe, M. Hirano, and H. Hosono, "Iron-Based Layered Superconductor La [O1-x F x] FeAs ( $\mathrm{x}=0.05-0.12)$ with T c= 26 K," J. Am. Chem. Soc., vol. 130, pp. 3296-3297, 2008.

[350] H. Kito, H. Eisaki, and A. Iyo, "Superconductivity at $54 \mathrm{~K}$ in F-Free NdFeAsO1-y," Journal of the Physical Society of Japan, vol. 77, p. 063707, 2008.

[351] M. Rotter, M. Tegel, and D. Johrendt, "Superconductivity at $38 \mathrm{~K}$ in the iron arsenide (Ba 1- x K x) Fe 2 As 2," Physi. Rev. Lett., vol. 101, p. 107006, 2008.

[352] H. Takahashi, K. Igawa, K. Arii, Y. Kamihara, M. Hirano, and H. Hosono, "Superconductivity at 43 [thinsp] K in an iron-based layered compound LaO1-xFxFeAs," Nature, vol. 453, pp. 376-378, 05/15/print 2008.

[353] C. Wang, L. Li, S. Chi, Z. Zhu, Z. Ren, Y. Li, Y. Wang, X. Lin, Y. Luo, and S. Jiang, "Thorium-doping-induced superconductivity up to $56 \mathrm{~K}$ in Gd1-xThxFeAsO," Europhys. Lett., vol. 83, p. 67006, 2008.

[354] R. Zhi-An, L. Wei, Y. Jie, Y. Wei, S. Xiao-Li, C. Guang-Can, D. Xiao-Li, S. Li-Ling, Z. Fang, and Z. Zhong-Xian, "Superconductivity at $55 \mathrm{~K}$ in iron-based F-doped layered quaternary compound Sm [O1-xFx] FeAs," Chin. Phys. Lett., vol. 25 , p. $2215,2008$.
[355]

H. Ogino, Y. Matsumura, Y. Katsura, K. Ushiyama, S. Horii, K. Kishio, and J.-i. Shimoyama, "Superconductivity at $17 \mathrm{~K}$ in (Fe2P2)(Sr4Sc2O6): a new superconducting layered pnictide oxide with a thick perovskite oxide layer," Supercond. Sci. Technol., vol. 22, p. 075008, 2009.

[356] X. Zhu, F. Han, G. Mu, P. Cheng, B. Shen, B. Zeng, and H.-H. Wen, "Transition of stoichiometric Sr 2 VO 3 FeAs to a superconducting state at 37.2 K," Physi. Rev. B, vol. 79, p. 220512, 2009.

[357] H. Ogino, Y. Shimizu, K. Ushiyama, N. Kawaguchi, K. Kishio, and J.-i. Shimoyama, "Superconductivity above $40 \mathrm{~K}$ observed in a new iron arsenide oxide (Fe2As2)(Ca4 (Mg, Ti) 3Oy)," Appl. Phys. Express, vol. 3, p. 063103, 2010.

[358] J. F. Ge, Z. L. Liu, C. Liu, C. L. Gao, D. Qian, Q. K. Xue, Y. Liu, and J. F. Jia, "Superconductivity above $100 \mathrm{~K}$ in single-layer FeSe films on doped SrTiO3," Nat. Mater., vol. 14, pp. 285-289, 2015.

[359] S. Parkin, V. Lee, E. Engler, A. Nazzal, T. Huang, G. Gorman, R. Savoy, and R. Beyers, "Bulk Superconductivity at $125 \mathrm{~K}$ in Tl $2 \mathrm{Ca}$ 2 Ba 2 Cu 3 O x," Physi. Rev. Lett., vol. 60, p. 2539, 1988.

[360] A. F. Hebard, M. J. Rosseinsky, R. C. Haddon, D. W. Murphy, S. H. Glarum, T. T. M. Palstra, A. P. Ramirez, and A. R. Kortan, "Superconductivity at $18 \mathrm{~K}$ in potassium-doped C60," Nature, vol. 350, pp. 600-601, 04/18/print 1991.

[361] M. Takigawa, A. Reyes, P. Hammel, J. Thompson, R. Heffner, Z. Fisk, and $\mathrm{K}$. Ott, "Cu and O NMR studies of the magnetic properties of YBa 2 Cu 3 O 6.63 (T c=62 K)," Physi. Rev. B, vol. 43, p. 247, 1991.

[362] T. Kaneko, H. Yamauchi, and S. Tanaka, "Zero-resistance temperature of Tl-based "2223" superconductor increased to 127 K," Phys. C: Supercond., vol. 178, pp. 377-382, 1991.

[363] Y. Maeno, H. Hashimoto, K. Yoshida, and S. Nishizaki, "Superconductivity in a layered perovskite without copper," Nature, vol. 372, p. 532, 1994.

[364] J. Schön, C. Kloc, and B. Batlogg, "Superconductivity at $52 \mathrm{~K}$ in hole-doped C60," Nature, vol. 408, pp. 549-552, 2000.

[365] J. Nagamatsu, N. Nakagawa, T. Muranaka, Y. Zenitani, and J. Akimitsu, "Superconductivity at 39 [thinsp]K in magnesium diboride," Nature, vol. 410, pp. 63-64, 2001.

[366] Z. A. Ren, J. Yang, W. Lu, W. Yi, X. L. Shen, Z. C. Li, G. C. Che, X. L. Dong, L. L. Sun, and F. Zhou, "Superconductivity in the ironbased F-doped layered quaternary compound Nd [O1- x Fx] FeAs," Europhys. Lett., vol. 82, p. 57002, 2008.

[367] A. Schilling, M. Cantoni, J. Guo, and H. Ott, "Superconductivity above $130 \mathrm{~K}$ in the Hg-Ba-Ca-Cu-O system," Nature, vol. 363, pp. 56-58, 1993.

[368] A. Drozdov, M. Eremets, I. Troyan, V. Ksenofontov, and S. Shylin, "Conventional superconductivity at 203 kelvin at high pressures in the sulfur hydride system," Nature, vol. 525, pp. 73-76, 2015.

[369] A. Drozdov, M. Eremets, and I. Troyan, "Conventional superconductivity at $190 \mathrm{~K}$ at high pressures," arXiv preprint arXiv:1412.0460, 2014.

[370] L. Gao, Y. Xue, F. Chen, Q. Xiong, R. Meng, D. Ramirez, C. Chu, J. Eggert, and H. Mao, "Superconductivity up to $164 \mathrm{~K}$ in $\mathrm{HgBa} 2$ $\mathrm{Ca} \mathrm{m}-1 \mathrm{Cu} \mathrm{m} \mathrm{O} 2 \mathrm{~m}+2+\delta(\mathrm{m}=1,2$, and 3$)$ under quasihydrostatic pressures," Physi. Rev. B, vol. 50, p. 4260, 1994.

[371] Z. H. Yuan, J. F. Feng, P. Guo, C. H. Wan, H. X. Wei, S. S. Ali, X. F. Han, T. Nakano, H. Naganuma, and Y. Ando, "Low frequency noise in magnetic tunneling junctions with Co40Fe40B20/Co70.5Fe4.5Si15B10 composite free layer," $J$. Magn. Magn. Mater., vol. 398, pp. 215-219, 1/15/ 2016.

[372] F. Guo, G. McKusky, and E. D. Dahlberg, "Absence of magnetic state dependent low-frequency noise in spin-valve systems," Physi. Rev. B, vol. 88, p. 014409, 07/09/ 2013.

[373] R. Stearrett, W. Wang, L. Shah, J. Xiao, and E. Nowak, "Magnetic noise evolution in $\mathrm{CoFeB} / \mathrm{MgO} / \mathrm{CoFeB}$ tunnel junctions during annealing," Appl. Phys. Lett., vol. 97, pp. 243502-243502-3, 2010.

[374] L. Jiang, E. Nowak, P. Scott, J. Johnson, J. Slaughter, J. Sun, and R. Dave, "Low-frequency magnetic and resistance noise in magnetic tunnel junctions," Physi. Rev. B, vol. 69, p. 054407, 2004.

[375] C. Ren, X. Y. Liu, B. D. Schrag, and G. Xiao, "Low-frequency magnetic noise in magnetic tunnel junctions," Physi. Rev. B, vol. 69, Mar 2004.

[376] A. Gokce, E. Nowak, S. H. Yang, and S. Parkin, "1/f noise in magnetic tunnel junctions with $\mathrm{MgO}$ tunnel barriers," J. Appl. Phys., vol. 99, pp. 08A906-08A906-3, 2006. 
A. F. M. Nor, T. Kato, S. J. Ahn, T. Daibou, K. Ono, M. Oogane, Y. Ando, and T. Miyazaki, "Low-frequency noise in $\mathrm{MgO}$ magnetic tunnel junctions," J. Appl. Phys., vol. 99, p. 08T306, 2006.

[378] F. G. Aliev, R. Guerrero, D. Herranz, R. Villar, F. Greullet, C. Tiusan, and M. Hehn, " Very low 1/f noise at room temperature in fully epitaxial $\mathrm{Fe} / \mathrm{MgO} / \mathrm{Fe}$ magnetic tunnel junctions," Appl. Phys. Lett., vol. 91, pp. 232504-232504-3, 2007.

[379] J. Scola, H. Polovy, C. Fermon, M. Pannetier-Lecoeur, G. Feng, K. Fahy, and J. Coey, "Noise in $\mathrm{MgO}$ barrier magnetic tunnel junctions with $\mathrm{CoFeB}$ electrodes: Influence of annealing temperature," Appl. Phys. Lett., vol. 90, pp. 252501-252501-3, 2007.

[380] Z. Diao, J. Feng, H. Kurt, G. Feng, and J. Coey, "Reduced low frequency noise in electron beam evaporated $\mathrm{MgO}$ magnetic tunnel junctions," Appl. Phys. Lett., vol. 96, p. 202506, 2010.

[381] G. Yu, Z. Diao, J. Feng, H. Kurt, X. Han, and J. Coey, "1/f noise in $\mathrm{MgO}$ double-barrier magnetic tunnel junctions," Appl. Phys. Lett., vol. 98, p. 112504, 2011.

[382] P. Wisniowski, M. Dąbek, W. Skowronski, T. Stobiecki, S. Cardoso, and P. P. Freitas, "Reduction of low frequency magnetic noise by voltage-induced magnetic anisotropy modulation in tunneling magnetoresistance sensors," Appl. Phys. Lett., vol. 105, p. 082404, 2014.

[383] P. Wisniowski, M. Dabek, and J. Wrona, "Field noise in tunneling magnetoresistance sensors with variable sensitivity," Appl. Phys. Lett., vol. 106, p. 052404, 2015.

[384] R. Araneo, G. Lovat, S. Celozzi, and P. Burghignoli, "Shielding effectiveness of finite width shields against low-impedance magnetic near-field sources," in the Intl. ACES, Denver, CO, USA, 2018

[385] Y. Nagasaki, M. Solovyov, and F. Gömöry, "Experimental and numerical investigation of shielding performance of superconducting magnetic shields using coated conductor tapes," IEEE Trans. Appl. Supercond., vol. 28, pp. 1-5, 2018.

[386] K. Livesey, R. Camley, Z. Celinski, and S. Maat, "Magnetic shielding of 3-phase current by a composite material at low frequencies," AIP Adv., vol. 7, p. 056328, 2017.

[387] L. Grimaud and S. Mazouffre, "Conducting wall Hall thrusters in magnetic shielding and standard configurations," J. Appl. Phys., vol. 122, p. 033305, 2017.

[388] K. Draxler and R. Styblíková, "Magnetic shielding of Rogowski coils," IEEE Trans. Instrum. Meas. , 2018.

[389] C. Bibirica, S. Cristian, L. Ene, and M. Iordache, "Improving the performance of PCB inductors for WPT systems using magnetic shields," in the ISEEE, Galati, Romania, 2017.

[390] J. I. Musher, "Linear variation of proton magnetic shielding with electric field," J. Chem. Phys., vol. 37, pp. 34-39, 1962.

[391] T. Sumner, J. Pendlebury, and K. Smith, "Convectional magnetic shielding," J. Phys. D: Appl. Phys., vol. 20, p. 1095, 1987.

[392] J. Hoburg, "Principles of quasistatic magnetic shielding with cylindrical and spherical shields," IEEE Trans. Electromagn. Compat., vol. 37, pp. 574-579, 1995.

[393] I. G. Mikellides, I. Katz, R. R. Hofer, D. M. Goebel, K. De Grys, and A. Mathers, "Magnetic shielding of the channel walls in a Hall plasma accelerator," Phys. Plasmas, vol. 18, p. 033501, 2011.

[394] W. Wadey, "Magnetic shielding with multiple cylindrical shells," Rev. Science Instrum., vol. 27, pp. 910-916, 1956.

[395] Z. Lei, G. Li, W. F. Egelhoff, P. Lai, and P. W. T. Pong, "Magnetic tunnel junction sensors with conetic alloy," IEEE Trans. Magn., vol. 47, pp. 714-717, 2011.

[396] J. Mead and J. Martin, "Improvement of resolution in large area photomultipliers," Nucl. Instrum. Methods, vol. 36, pp. 13-22, 1965.

[397] V. Lapkovskis, V. Mironovs, I. Jevmenov, A. Kasperovich, and V. Myadelets, "Multilayer material for electromagnetic field shielding and EMI pollution prevention," Agronomy Research, vol. 15, pp. 1067-1071, 2017.

[398] J. Joo and C. Lee, "High frequency electromagnetic interference shielding response of mixtures and multilayer films based on conducting polymers," J. Appl. Phys., vol. 88, pp. 513-518, 2000.

[399] S.-M. Yang, Y. Chang, Y. Hsieh, and Y. Lee, "Electromagnetic shielding effectiveness of multilayer metallic thin film on plastic substrates," J. Appl. Polym. Sci., vol. 110, pp. 1403-1410, 2008.

[400] P. L. Sergeant, L. R. Dupré, M. De Wulf, and J. A. Melkebeek, "Optimizing active and passive magnetic shields in induction heating by a genetic algorithm," IEEE Trans. Magn., vol. 39, pp. 3486-3496, 2003
[401] E. Donley, E. Hodby, L. Hollberg, and J. Kitching, "Demonstration of high-performance compact magnetic shields for chip-scale atomic devices," Rev. Science Instrum., vol. 78, p. 083102, 2007.

[402] T. Kornack, S. Smullin, S.-K. Lee, and M. Romalis, "A low-noise ferrite magnetic shield," Appl. Phys. Lett., vol. 90, p. 223501, 2007.

[403] J. Kvitkovic, S. Patel, and S. Pamidi, "Magnetic Shielding Characteristics of Hybrid High-Temperature Superconductor/Ferromagnetic Material Multilayer Shields," IEEE Trans. Appl. Supercond., vol. 27, pp. 1-5, 2017.

[404] F. Wang, X. Yang, X. Liu, T. Niu, J. Wang, Z. Mei, and Y. Jian, "Design of an ultra-thin absorption layer with magnetic materials based on genetic algorithm at the S band," J. Magn. Magn. Mater., vol. 451, pp. 770-773, 2018.

[405] A. Canova, J. C. del-Pino-López, L. Giaccone, and M. Manca, "Active shielding system for elf magnetic fields," IEEE Trans. Magn., vol. 51, pp. 1-4, 2015.

[406] I. Briki, L. Pichon, and J. B. H. Slama, "Shielding Effectiveness of Perforated Screens Through an Inverse Problem-Based Resolution," IEEE Trans. Magn., vol. 52, pp. 1-4, 2016.

[407] P. Wisniowski, M. Dąbek, W. Skowronski, T. Stobiecki, S. Cardoso, and P. Freitas, "Reduction of low frequency magnetic noise by voltage-induced magnetic anisotropy modulation in tunneling magnetoresistance sensors," Appl. Phys. Lett., vol. 105, p. $082404,2014$.

[408] X. Liu, K. Lam, K. Zhu, C. Zheng, X. Li, Y. Du, C. Liu, and P. W. T. Pong, "Overview of Spintronic Sensors, Internet of Things, and Smart Living," arXiv preprint arXiv:1611.00317, 2016.

[409] E. E. Fullerton and J. R. Childress, "Spintronics, magnetoresistive heads, and the emergence of the digital world," Proc. IEEE, vol. 104, pp. 1787-1795, 2016.

[410] M.Y. Cheng, C.M. Tsao, Y. Z. Lai and Y. J. Yang, "The development of a highly twistable tactile sensing array with stretchable helical electrodes," Sens. Actuators, A., vol. 166, pp.226233, 2011.

[411] V. C. Martins, J. Germano, F. A. Cardoso, J. Loureiro, S. Cardoso, L. Sousa, M. Piedade, L. P. Fonseca, and P. Freitas, "Challenges and trends in the development of a magnetoresistive biochip portable platform," J. Magn. Magn. Mater., vol. 322, pp. 1655-1663, 2010.

[412] S. Cardoso, D. Leitao, T. Dias, J. Valadeiro, M. Silva, A. Chicharo, V. Silverio, J. Gaspar, and P. Freitas, "Challenges and trends in magnetic sensor integration with microfluidics for biomedical applications," J. Phys. D: Appl. Phys., vol. 50, p. $213001,2017$.

[413] G. Lin, D. Makarov, and O. G. Schmidt, "Magnetic sensing platform technologies for biomedical applications," Lab Chip, vol. 17, pp. 1884-1912, 2017.

[414] M. Adnan, D. Hammerschmidt, and M. Huemer, "Phase demodulation of magnetic GMR signal for virtual sensing applications by using hilbert transform," in the $38^{\text {th }} M I P R O$, Opatija, Croatia, 2015

[415] M. Erol-Kantarci and H. T. Mouftah, "Suresense: sustainable wireless rechargeable sensor networks for the smart grid," IEEE Wireless Commun. Lett., vol. 19, 2012.

[416] P. Guo, X. Liu, S. Tang, and J. Cao, "Concurrently wireless charging sensor networks with efficient scheduling," IEEE Trans. Mob. Comput., vol. 16, pp. 2450-2463, 2017.

[417] S. R. Samantaray, I. Kamwa, and G. Joos, "Phasor measurement unit based wide-area monitoring and information sharing between micro-grids," IET Gener. Transm. Distrib., vol. 11, pp. 1293-1302, 2017.

[418] R. Ghiga, K. Martin, Q. Wu, and A. H. Nielsen, "Phasor measurement unit test under interference conditions," IEEE Trans. Power Del., vol. 33, pp. 630-639, 2018.

[419] LORD Sensing, "3DM-GX5-35 Attitude and Heading Reference System (AHRS) with GNSS", 2007. Available: http://www.microstrain.com/inertial/3dm-gx5-35. [Accessed: Sep16-2018].

[420] Honeywell, "Versatile, reliable and accurate navigation", 2002. Available:

https://aerospace.honeywell.com/en/products/navigation-andsensors/talin-marine-inertial-navigation-system. [Accessed: Sep16-2018].

[421] F. Bonomi, R. Milito, J. Zhu, and S. Addepalli, "Fog computing and its role in the internet of things," in Proceedings of the first edition of the MCC workshop on Mobile cloud computing, 2012, pp. 13-16. 
J. Gubbi, R. Buyya, S. Marusic, and M. Palaniswami, "Internet of Things (IoT): A vision, architectural elements, and future directions," Future Gener. Comput. Syst., vol. 29, pp. 1645-1660, 2013.

[423] L. Atzori, A. Iera, and G. Morabito, "The internet of things: A survey," Comput. Networks, vol. 54, pp. 2787-2805, 2010.

[424] M. Swan, "Sensor mania! the internet of things, wearable computing, objective metrics, and the quantified self $2.0, " \mathrm{~J}$. Sens. Act. Networks, vol. 1, pp. 217-253, 2012.

[425] D. Lymberopoulos, A. Bamis, and A. Savvides, "Extracting spatiotemporal human activity patterns in assisted living using a home sensor network," Universal Access Inform. Soc., vol. 10, pp. 125-138, 2011.

[426] J. Bohn, V. Coroamă, M. Langheinrich, F. Mattern, and M. Rohs, "Living in a world of smart everyday objects - social, economic, and ethical implications," Human Ecological Risk Asses., vol. 10, pp. 763-785, 2004.

[427] P. Lombardi, S. Giordano, H. Farouh, and W. Yousef, "Modelling the smart city performance," Inno.: Europ. J. Soc. Sci. Research, vol. 25, pp. 137-149, 2012.

[428] C. Perera, A. Zaslavsky, P. Christen, and D. Georgakopoulos, "Sensing as a service model for smart cities supported by internet of things," Trans. Emerging Telecommun. Technol., vol. 25, pp. 81-93, 2014.

[429] V. R. Jakkula and D. J. Cook, "Detecting Anomalous Sensor Events in Smart Home Data for Enhancing the Living Experience," Artif. Intell. Smarter Living, vol. 11, p. 07, 2011.

[430] D. Ding, R. A. Cooper, P. F. Pasquina, and L. Fici-Pasquina, "Sensor technology for smart homes," Maturitas, vol. 69, pp. 131136, 2011.

[431] D. Basu, G. Moretti, G. S. Gupta, and S. Marsland, "Wireless sensor network based smart home: Sensor selection, deployment and monitoring," in SAS, Galveston, TX, USA, 2013.

[432] D. J. Cook and L. B. Holder, "Sensor selection to support practical use of health-monitoring smart environments," Wiley Interdiscip. Rev.: Data Min. Knowl. Discovery, vol. 1, pp. 339-351, 2011.

[433] P. Tang and T. Venables, "'Smart'homes and telecare for independent living," Journal of Telemedicine and Telecare, vol. 6, pp. 8-14, 2000.

[434] C. Zhu and W. Sheng, "Wearable sensor-based hand gesture and daily activity recognition for robot-assisted living," IEEE Trans. Syst. Man Cybern. Part A Syst. Humans, vol. 41, pp. 569-573, 2011.

[435] J. Lee, H.-J. Kim, G.-L. Park, and H. Jeon, "Genetic algorithm-based charging task scheduler for electric vehicles in smart transportation," Int. J. Intell. Inf. Database Syst., pp. 208-217, 2012.

[436] L. Schewel and D. M. Kammen, "Smart transportation: Synergizing electrified vehicles and mobile information systems," Environment, vol. 52, pp. 24-35, 2010.

[437] M. A. Kumbhar, "Wireless sensor networks: A solution for smart transportation," J. Emerging Trends in Comput. Inform. Sci., vol. 3, 2012.

[438] G. Stefansson and K. Lumsden, "Performance issues of smart transportation management systems," Int. J. Productivity Performance Management, vol. 58, pp. 55-70, 2008.

[439] Zettlex, "Magnetic position sensors", 2018. Available: https://buff.ly/2pRh6TO. [Accessed: Sep-6-2018]

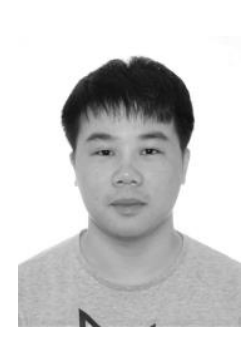

Chao Zheng was born in Zhejiang, China in 1990. He received the B.S. degree in Applied Physics from Zhejiang University of Technology, Hangzhou, Zhejiang, China, in 2013. He is currently pursuing the Ph.D. degree in Electrical and Electronic Engineering at the University of Hong Kong, Hong Kong.

His research interests include development of highlysensitive magnetic tunnel junction sensors for ultralow-fieldsensing applications, fundamental study of magnetic lowfrequency noise in magnetic tunnel junction sensors, and magnetic thin films. Dr. Zheng is a member of IEEE Magnetics Society (Hong Kong Chapter).

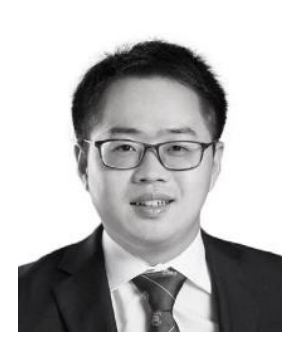

Ke Zhu was born in Yichang, China in 1990. He received the B.Eng. degree in Electrical Engineering from China Three Gorges University (CTGU), China, in 2013. He obtained the Ph.D. degree in Electrical and Electronic Engineering at the University of Hong Kong (HKU) in 2018, and now continues as a postdoctoral researcher. His current research and academic interests focus on computational electromagnetics, electric power transmission monitoring, and application of magnetoresistive (MR) sensors in smart grid. Dr. Zhu is a member of IEEE Magnetics Society (Hong Kong Chapter).

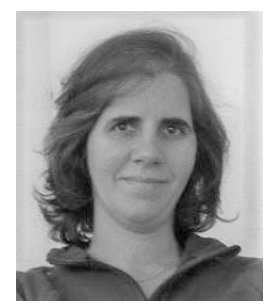

Susana Cardoso de Freitas was born in Lisbon, Portugal in 1973. She received the B.S. and M.S. degrees in Technological Physics Engineering from the Instituto Superior Tecnico, University of Lisbon in 1996 and the Ph.D. degree in Physics from Instituto Superior Tecnico, in 2002.

In 2002 she was a "Co-op Pre-Professional Engineer" at IBM, T. J. Watson Research Center (USA). Since 2002 she is a researcher at INESC-MN and the co-leader of the Magnetics \& Spintronics group since 2006. She is an Associated Professor at the Physics Department (IST) since 2015, and is responsible for student coordination (over 46 master students, $4 \mathrm{PhD}$ students and 10 post-doctoral fellows). She is co-author of over 260 publications (researcher ID: B-6199-2013). Her research interests include advanced thin films, spintronic sensors, microfabrication processes in large area wafers, and sensors for robotics, biomedical and industrial applications.

Dr. Cardoso de Freitas was a recipient of the Honorable Mention in Scientific Awards Universidade de Lisboa/Santander in 2016 and 2017, and the Magnetic Society of Japan Distinguished Publication Award for the book edited: Giant Magnetoresistance (GMR) Sensors, Ed. Springer, in 2014, and she was one of the team members awarded as 2nd finalist for the EU Descartes Prize for Research in 2004. 


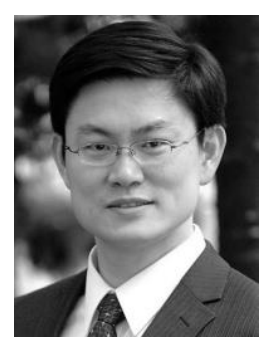

Jen-Yuan (James) Chang was born in Taipei, Taiwan in 1972. He received B.S. degree in 1994 from National Central University, Taiwan and M.S. and Ph.D. degrees in 1998 and 2001, respectively from Carnegie Mellon University, USA, all in Mechanical Engineering.

$\mathrm{He}$ is currently a Professor in the Department of Power Mechanical Engineering, National Tsing Hua University, Taiwan. Following his Assistant Professorship with Washington State University, USA, he served a Senior Lecturer in Massey University, New Zealand. He was an ASEE/NRC Faculty Research Fellow at AFRL, USAF. He holds Visiting Scholar/Professor positions at Hiroshima University, Japan, at Data Storage Institute, A*STAR, Singapore, at Institute of Biomedical Technologies, Auckland University of Technology, New Zealand and at Yonsei University, Korea. From 2001-2006, he worked at various R\&D positions for high-end magnetic disk storage devices with IBM and HGST in San Jose, California, USA.

Dr. Chang was a recipient of ISPS Outstanding Contribution Award, Professor Chang, an ASME Fellow. He was the Division Chair of ISPS Division, Vice Chair of Strategic Planning Committee, and Member of Technical Committee of IEEE Magnetics Society. Professor Chang have served as TE and AE of IEEE/ASME Transactions on Mechatronics, ASME Journal of Vibration and Acoustics, and Springer Microsystems Technologies.

Joseph E. Davies received his B.S. degree in Physics and Mathematics from Hamline University, located in St. Paul, MN in 2000 and his Ph.D. degree in Physics from the University of California, Davis, in 2007.

From 1998 to 2000, he worked in the Microreplication Technology Center at 3M. From 2000 to 2001, he was as a Software Engineer for Honeywell's commercial aviation products division. From 2002 to 2007, he worked as a graduate student researcher working on magnetic thin-films and nanostructures. From 2007 to 2009, he continued his magnetic materials work as a National Research Council postdoctoral fellow at the National Institute of Standards and Technology in Gaithersburg, Maryland. Since 2009, he has worked as a physicist and program manager for NVE Corporation in Eden Prairie, MN. His activities include the development of magnetic tunnel junction sensor materials and devices as well as the development and characterization of magnetoelastic devices for

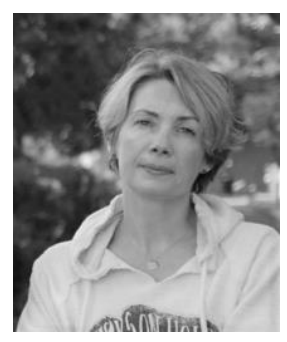

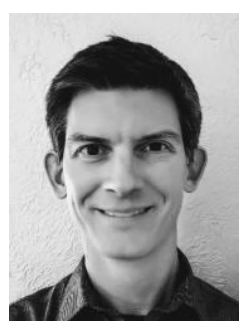

Peter Eames received his Ph.D. in experimental condensed matter physics from the University of Minnesota in 2003 and B.S. degrees in physics in 1998, graduating cum laude. Both degrees were earned at the University of Minnesota in Minneapolis, MN USA.

He is currently the Vice President of Advanced Technology at NVE Corporation in Eden Prairie, MN where he drives excellence in innovation in magnetic devices including NVE's AMR, GMR, and TMR magnetic sensors and isolators.

Dr. Eames has been a member of the American Physics Society since 2010 . He is currently a member of the IEEE and has been a member of the IEEE technical committee on magnetism since 2014 .

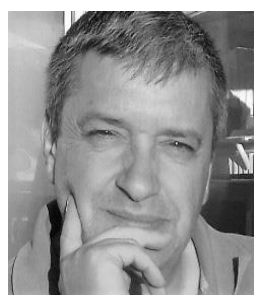

Paulo P. Freitas was born in Lisbon, Portugal in 1958. He received the B.S. and M.S. degrees in Physics from the University of Porto in 1981 and the Ph.D. degree in Physics from Carnegie Mellon University, Pittsburgh, USA, in 1986.

After a postdoctoral appointment at IBM T.J. Watson Research Center, USA, in 1988 he joined INESC where he created the Solid State Technology Group. He is full professor of Physics at Instituto Superior Tecnico. Since 2008 he is Deputy Director General of the International Iberian Nanotechnology Laboratory. His research activities include

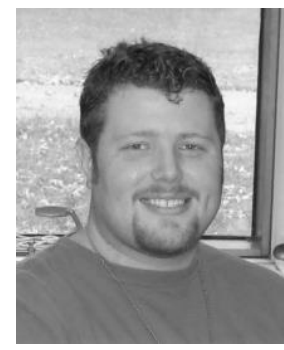
spintronics and applications in sensing, memory and biomedical applications. He has published 450 research articles, several patents, advised $20 \mathrm{PhD}$ students, and participated in a start-up using INESC MN biochip technology.

Dr. Freitas was a recipient of the Gulbenkian Foundation Nanotechnology Award in 2004, the Portuguese Foundation for Science and Technology Excellence Award in 2006, and received the Scientific Award of the Technical University of Lisbon in 2008. He was one of the team members awarded as $2^{\text {nd }}$ finalist for the EU Descartes Prize for Research in 2004. Within the IEEE Magnetics Society, he is a senior member since 2016. He was elected for IEEE Mag Soc Procom in 2007 and a Distinguished lecturer in 2008. Since 2016 he is one effective member of the Portuguese Academy of Sciences.

Olga Kazakova was born in Tambov, Russia. She received the Ph.D. degree in Solid State Physics from Institute of Crystallography, Russian Academy of Science in 1996.

In 1999-2001, she was a Postdoctoral Researcher at Chalmers University of Technology, Gothenburg, Sweden. Later on in 2001-2002, she was an Assistant Professor there, working on magnetic nanostructures and their applications. 
Since 2002, she has been working at the National Physical Laboratory, where she became a Principle Research Scientist in 2010. Her research interests include functional (electronic, optical, structural) nanoscale studies of 2D materials; novel environmental sensors based on 2D materials; novel sensors for life Science and food industries; magnetic nanosensors for biological and metrological applications. She is an author of above 140 peer-refereed publications and had above 130 presentations at scientific conferences, e.g., above 50 invited talks and seminars.

Dr. Kazakova was a recipient of the numerous national and international awards, including Intel European Research and Innovation Award (2008), NPL Rayleigh Award and Serco Global Pulse Award (2011). She is a Fellow of Institute of Physics.

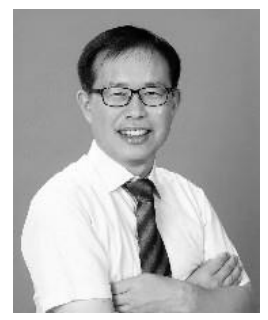

CheolGi Kim received the B.S. degree in Physics from Seoul National University, Korea in 1983, and his M.S. and Ph.D. degrees from KAIST, Korea in 1986 and 1989, respectively. He was a Postdoctoral Researcher at NIST in USA.

Prior to coming to DGIST in 2014, he has 24 years of research experience at KRISS and Chungnam National University in Korea, Tohoku (Japan), MaMaster (Canada) and Bielefeld (Germany) Universities. His research interests skate the intersection between nano-spintronics and bio-medical Sciences. During his Professional period, he published 360 reputed articles, and 25 patents. Especially, he is the Director of "Center for Bio-Convergence Spin System", directed to the bio-initiative spintronics device development.

Professor Kim is currently a Professor and Dean of the Graduate School at DGIST, and Co-Director of VNU key laboratory, Vietnam. He has honored by 8 awards from domestic academic societies, and Distinction medal from Montpellier University in France. He has served as the General Secretary of Asian Union of Magnetic Societies during 20162017.

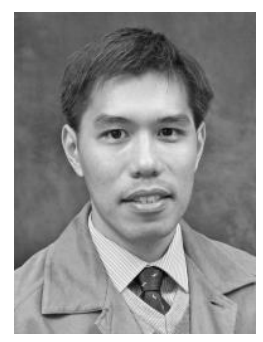

Chi-Wah (Dennis) Leung received the B.Eng. degree in Mechanical Engineering from the University of Hong Kong, China, in 1999 and the Ph.D. degree in Materials Science from the University of Cambridge, U.K., in 2003.

From 2003 to 2005, he was a Postdoctoral Fellow in the Materials Science Department, the University of Cambridge. In 2005 he joined the Department of Applied Physics, the Hong Kong Polytechnic University as a Lecturer. Since 2015 he has been an Associate Professor of the Department. He is the author of more than 140 articles. His research interests include thin films and devices structures for electrical, spintronic and photonic applications, and fabrication of micro- or nanostructured surfaces.

Dr. Leung is the Chairperson of the IEEE Magnetics Society (Hong Kong Chapter) since 2015. He was an Editor for the Joint MMM-Intermag 2016 and Intermag 2017.

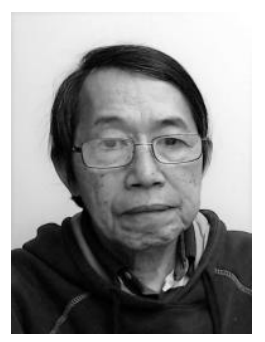

Sy-Hwang Liou received the B.S. degree in Physics from Soochow University, Taiwan, in 1974, the M.S. degree in Physics from Florida Institute of Technology, Melbourne, FL, and the M.A. and Ph.D. degrees in Physics from the Johns Hopkins University, Baltimore, MD, in 1981 and 1985, respectively.

He joined the Department of Physics and Astronomy, University of Nebraska, Lincoln, NE, in 1988 as an Assistant Professor and is currently a Professor of Physics. He is a Guest Research Scientist with the National Institute of Standards and Technology, CO since 1995. His research includes a variety of interests in the fields of magnetic interactions, domain images, nanofabrication, magnetic characterization of nanostructures by magnetic force microscopy, microcantilever torque magnetometry, and magnetic sensors.

Dr. Liou served as a Co-Editor of Applied Physics Communications from 1990 to 1994, Member of IEEE Magnetic Society Technical Committee (2005-2008), Member of IEEE Intermag 2009 program committee, Member of Editorial Review Board IEEE Magnetic Letters (2009-2012), Member of program committee of 13th Joint MMM-Intermag Conference (2016), and is a member of the American Physical Society, Materials Research Society, and IEEE.

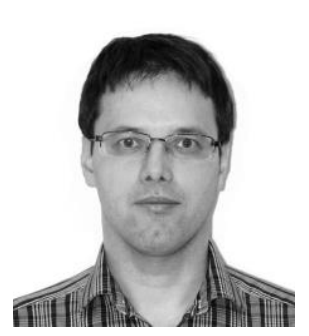

Alexey Ognev received the M.S. degree in Physics in 2000 and Ph.D. degree in Condensed Matter Physics in 2004 and Doctor of Science degree in 2017 from Far Eastern Federal University, Vladivostok, Russia. He is currently on the position of Head in Laboratory of Thin Film Technologies of Far Eastern

Federal University.

In 2007 he worked as a Researcher in Freie Universität, Berlin, Germany. In 2007 and 2013 he worked as a Researcher in NanoScience group, University of Bath, UK. He is the author of 70 articles and 10 inventions. His research interests include magnetic anisotropy and domain structures, nanomagnetism, spintronics, spin-orbitronics, and sensorics. 
Dr. Ognev's awards include the Best Young Lecturer of Russia, Gold Medal of the Seoul International Invention Fair.

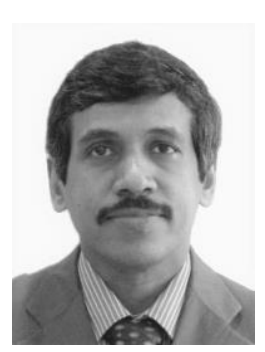

S. N. Piramanayagam was born in Tirunelveli, India in 1965. He got his M.Sc. degree in Physics from University of Kerala, Trivandrum, India in 1988. He got his Ph.D. degree from Indian Institute of Technology, Bombay, India in 1994. He has been working in the field of magnetic materials for the past $25+$ years. His current research focus is on magnetism and nanostructures for spintronics, energy and biomedical applications.

He worked in Data Storage Institute, Singapore from 1999 to 2015. During this period, he served in various capacities, such as Senior Research Engineer, Assistant Program Manager, Principal Research Engineer, Research Scientist and Senior Scientist. He also served as an Adjunct Associate Professor at National University of Singapore during 2003-2009. During this period, he co-supervised $10 \mathrm{PhD}$ students and 2 Masters Students. Currently, he is serving as an Associate Professor in Nanyang Technological University, Singapore. He has more than 150 publications in ISI journals. He has published a book on "Developments in Data Storage: Materials Perspective" under IEEE-Wiley Press.

Prof. Piramanayagam is a member of IEEE and IEEE Magnetics Society. He was the Chair of Technical Committee of IEEE Magnetics Society from 2013-2016. He was also the Chair of IEEE Magnetics Society, Singapore Chapter for many years.

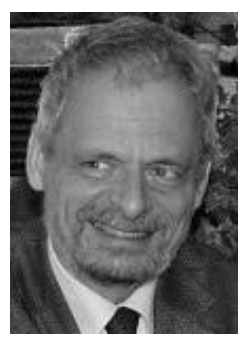

Pavel Ripka was born in Praha, Czech Republic in 1959. He received an Ing. degree in Electrical Engineering in 1984, and CSc. (equivalent to Ph.D.) in 1989. In 1996 he received docent (Assoc. Prof.) degree.

He has been working at the Department of Measurement, Faculty of Electrical Engineering, Czech Technical University (CTU) since 1990. In 1990-1994 he was a visiting researcher at the Danish Technical University. In 2001 he was a Marie Curie Advanced Researcher Fellow at University of Galway, Ireland. In 2005-2006: he was a Visiting Scientist at the Institute for the Protection and the Security of the Citizen, Ispra, Italy. Now he is a full Professor at CTU, lecturing in Measurements, Engineering Magnetism and Sensors. His main research interests are magnetic measurements and magnetic sensors, especially fluxgate. He is a co-author of 3 books and 138 journal papers. He also participates in industrial research and holds 12 patents.

Pavel Ripka was an Associated Editor of IEEE Sens. J., and a member of the Editorial Boards of Technisches Messen, Meas. Sci. Technol., and Journal of Sensors. He has been a member of the Eurosensors Steering Committee and Program Committees of IEEE Intermag and IEEE Sensors conferences.

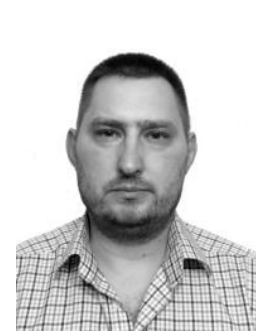

Alexander Samardak received the M.S. degree in Physics in 2000 and Ph.D. degree in Condensed Matter Physics in 2004 from Far Eastern Federal University, Vladivostok, Russia. He is currently on the positions of Associate Professor and Leading Researcher in Laboratory of Thin Film Technologies of Far Eastern Federal

University.

From 2006 to 2009, he was a Research Officer in NanoScience group, University of Bath, UK. In 2009 he worked as a Researcher in Norwegian University of Science and Technology, Trondheim. In 2010 he was a Researcher with Department of Physics, Muenster University, Germany. In 2017 he had a position of Research Professor in Center for SpinOrbitronic Materials, Korea University, Seoul, Korea. He is the author of 75 articles and 10 inventions. His research interests include nanomagnetism, spintronics, spin-orbitronics, sensorics and artificial intelligence. $\mathrm{He}$ is an Associate Editor of the journal Advances in Nano Research.

Dr. Samardak's awards include the Science Drive Program of Nobel Laureate Andre Geim Award, the Best Young Scientist of Russia, Gold Medal of the Seoul International Invention Fair, Gold Medal in the International Competition of Young Scientists in Nanotechnologies (RusnanoForum).

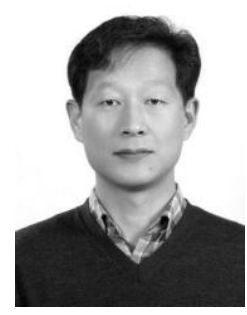

Kwang-Ho Shin was born in Daegu, Korea in 1970. He received his B.S. and M.S. degrees in Electrical Engineering from Dong-A University, Busan, Korea, in 1993 and 1995 and the Ph.D. degree in Electrical Telecommunication Engineering from Tohoku University, Sendai, Japan, in 1999.

From 1999 to 2000, he had worked for Samsung Advanced Institute of Technology (SAIT). He has been with Kyungsung University as a Professor in the Department of Information Communication Engineering since September 2000. From 2006 to 2007, he had been an Associate Professor of Toyohashi University of Technology, Japan. He was the Chief Technical Officer of CNK Co. from 2001 to 2003, and the Technical Advisor of Shilla Industrial Co. from 2015 to 2017 . He is the author of three books, more than 100 technical papers in the research field of micro magnetic devices, high frequency devices and sensor engineering. 
Dr. Shin is an Editor of the Journal of Magnetics. His awards and honors include the Takei Award from the Japanese Magnetics Society and Best Paper Award from the Korean Magnetics Society. He served for Korean government as a member of Smart Sensor Development Strategy Committee, 2014 and IoT Sensor Roadmap Committee, 2015.

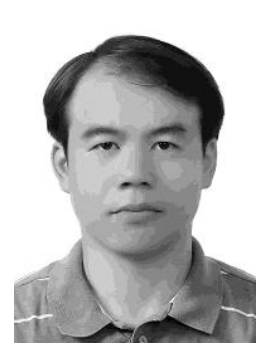

Shi-Yuan Tong was born in Taichung, Taiwan in 1977. He received the B.S. degree of Materials Science and Engineering from Feng Chia University in 1999, the M.S. degree from National Taiwan University in 2001 and the Ph.D. degree in Materials Science and Engineering from National Tsing Hua University in 2013.

He worked as a Research Assistant in Center for Condensed Matter Sciences in Taipei for a short time and since 2002, he starts joining the Electromagnetic Material and Device Lab. which located at Industrial Technology Research Institute in Hsinchu. He is a researcher working on the circuit design, device fabrication and measurement integration of highfrequency magnetic materials which can be applied in the field of power modules, electromagnetic suppressing and sensors. He has published more than 8 journal papers, 3 patents and some research results have been applied into the industry.

Dr. Tong is a member of Taiwan Association and Magnetic Technology and he also received the best essay award from Taiwan Materials Society in 2016.

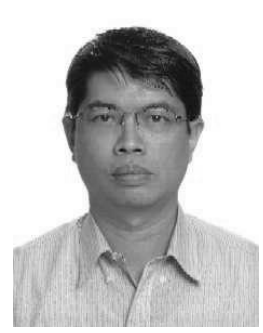

Mean-Jue Tung received the Bachelor's and Master's degree in Chemistry from the Chung Yuan Christian University Taiwan in 1983 and 1985, respectively, and the $\mathrm{Ph} . \mathrm{D}$. in Electronics Engineering from the National Chiao Tung University/Hsinchu in 2000 .

Dr. Tung entered the Taiwan Industrial Technology Research Institute (ITRI) in 1987 and engaged in magnetic materials research. He is currently a Principal Researcher at Materials and Chemical Research Institute/ITRI as the Deputy Director of the Electronic Materials Research Department and Supervisor of the Division of Electromagnetic Material and Components. The research topics are the development of soft magnetic materials, sensors and components, MRAM related topics and power electronics applications. He filed a total of 45 patents, has 10 patents for licensing and sale, published 134 papers. Most of the research results were transferred to Taiwanese companies for mass production.

Dr. Tung now serves as the President of the Taiwan Magnetic Technology Association (TAMT) and Council Member of the
Asian Union of Magnetic Societies (AUMS). He served as a Program Co-Chair of the international conferences Intermag 2011, ÍSAMMA 2013, and IcAUMS 2014.

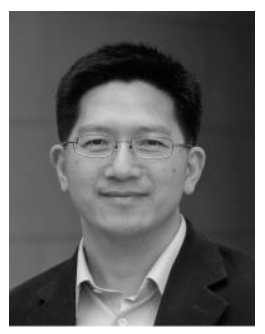

Shan X. Wang (M'88-SM'06-F'09) received the B.S. degree in Physics from the University of Science and Technology of China, Hefei, China, in 1986, the M.S. degree in Physics from Iowa State University, Ames, IA, USA, in 1988, and the Ph.D. degree in Electrical and Computer Engineering from Carnegie Mellon University, Pittsburgh, PA, USA, in 1993.

He currently serves as a Professor and Associate Chair of Materials Science \& Engineering and jointly Professor of Electrical Engineering at Stanford University, and by courtesy, a Professor of Radiology at Stanford School of Medicine. He directs the Center for Magnetic Nanotechnology, and is a CoPrincipal Investigator of the Center for Cancer Nanotechnology Excellence for Translational Diagnostics (CCNE-TD) at Stanford University. He has over 270 publications, and holds 56 issued or pending patents in the areas of magnetic nanotechnology, biosensors, nanofabrication, spintronics, power management, and information storage.

Dr. Wang was elected a Fellow of the Institute of Electrical and Electronics Engineers (IEEE, 2009) and a Fellow of American Physical Society (APS, 2012) for his seminal contributions to magnetic materials and nanosensors. He has received numerous other awards, including most recently the Bold Epic Innovation (BEI) Award in the XPRIZE Qualcomm Tricorder Competition (April, 2017).

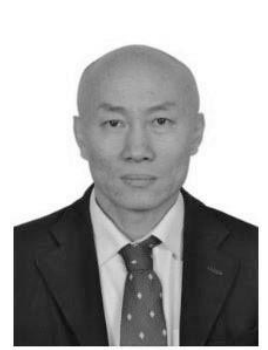

Songsheng Xue was born in China, in 1964. He received the B.S. degree in Optical Instrumentation from Zhejiang University, Hangzhou, China, in 1985, and the Ph.D. degree in Material Science from the Chinese Academy of Sciences in Shanghai, China, in 1991.

He engaged in the postdoctoral research in Physics at Laval University, Canada, in 1992. From 1994 to 1995, He was a Research Assistant with the Department of ECE at Carnegie Mellon University, USA. From 1995 to 2010, He worked in Seagate Technology, Oplink Communication, Honeywell and held positions as Engineer, Manager and Director.

In 2010, Dr. Xue founded MultiDimension Technology Corporation in Zhangjiagang city, Jiangsu, China, and held position as the President and CEO of the company since then. $\mathrm{He}$ is the author of more than 30 articles, and the inventor for more than 100 inventions. 


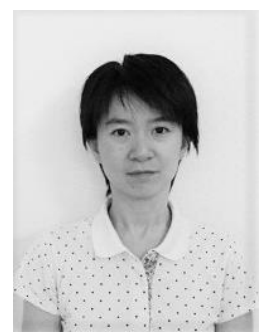

Xiaolu Yin was born in Changchun, Jilin, China. She received the B.S. degree from Optical Information Science and Technology, Changchun University of Science and Technology, Changchun, China, in 2008, and the Ph.D. degrees in Material Engineering, from the University of Nebraska, Lincoln, in May 2014. Her dissertation is "Tuning Magnetic Nanostructures for HighPerformance Magnetoresistive Sensors". She was a Postdoctoral Research Associate at the University of Nebraska, Lincoln from 2014 to 2015. She worked at Magnetic Imaging Group, Applied Physics Division, National Institute of Standards and Technology (NIST), Boulder, CO. from 2015 to 2017 as a Research Associate. She is currently a Staff Engineer at Western Digital Corporation, 44100 Osgood Rd. Fremont, CA. Her research interests include magnetic interactions in nanostructures, applications of nanostructures in magnetic sensors, development of ultrasensitive MEMS devices for the characterization of nanostructures, development of an ultra-low field Magn. Reson. Imaging (ulf-MRI) system for bio-medical applications.

Dr. Yin served as a member of IEEE Magnetic Society Technical Committee: Sensor Roadmap Taskforce initiation and discussion group, member of IEEE Intermag 2018 program committee, and is a member of the IEEE Society, and IEEE Magnetism Society.

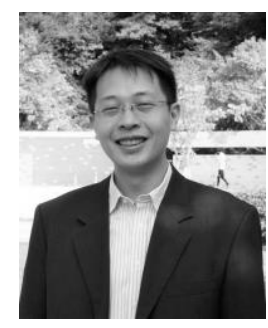

Philip W. T. Pong received a B.Eng. in Electrical and Electronic Engineering from the University of Hong Kong (HKU) with 1st class honours in 2002 and a Ph.D. degree in Engineering from the University of Cambridge in 2005.

After working as a Postdoctoral Researcher at the Magnetic Materials Group at the National Institute of Standards and Technology (NIST) in the United States for three years, he joined the HKU Department of Electrical and Electronic Engineering in 2008 where he is now an Associate Professor working on development of magnetoresistive (MR) sensors, and the applications of MR sensors in smart grid and smart living. He is serving on the Administrative Committee of the IEEE Magnetics Society.

Dr. Pong is a chartered physicist, chartered energy engineer, registered professional engineer, a fellow of the Institution of Engineering and Technology, a fellow of the Institute of Materials, Minerals and Mining, a fellow of the NANOSMAT Society, a senior member of IEEE, and a corporate member of Hong Kong Institution of Engineers (HKIE) in Electrical Division, Electronics Division and Energy Division. He serves as an editorial board member for three SCI journals. He was the recipient of the HKIE Young Engineer of the Year Award in 2016. 